\title{
Prioritizing the HIV response
}

Citation for published version (APA):

Safarnejad, A. (2017). Prioritizing the HIV response: a multi-criteria decision analysis. [Doctoral Thesis, Maastricht University]. Boekenplan. https://doi.org/10.26481/dis.20171123as

Document status and date:

Published: 01/01/2017

DOI:

10.26481/dis.20171123as

Document Version:

Publisher's PDF, also known as Version of record

\section{Please check the document version of this publication:}

- A submitted manuscript is the version of the article upon submission and before peer-review. There can be important differences between the submitted version and the official published version of record.

People interested in the research are advised to contact the author for the final version of the publication, or visit the DOI to the publisher's website.

- The final author version and the galley proof are versions of the publication after peer review.

- The final published version features the final layout of the paper including the volume, issue and page numbers.

Link to publication

\footnotetext{
General rights rights.

- You may freely distribute the URL identifying the publication in the public portal. please follow below link for the End User Agreement:

www.umlib.nl/taverne-license

Take down policy

If you believe that this document breaches copyright please contact us at:

repository@maastrichtuniversity.nl

providing details and we will investigate your claim.
}

Copyright and moral rights for the publications made accessible in the public portal are retained by the authors and/or other copyright owners and it is a condition of accessing publications that users recognise and abide by the legal requirements associated with these

- Users may download and print one copy of any publication from the public portal for the purpose of private study or research.

- You may not further distribute the material or use it for any profit-making activity or commercial gain

If the publication is distributed under the terms of Article $25 \mathrm{fa}$ of the Dutch Copyright Act, indicated by the "Taverne" license above, 


\section{Prioritizing the HIV Response}

A multi-criteria decision analysis

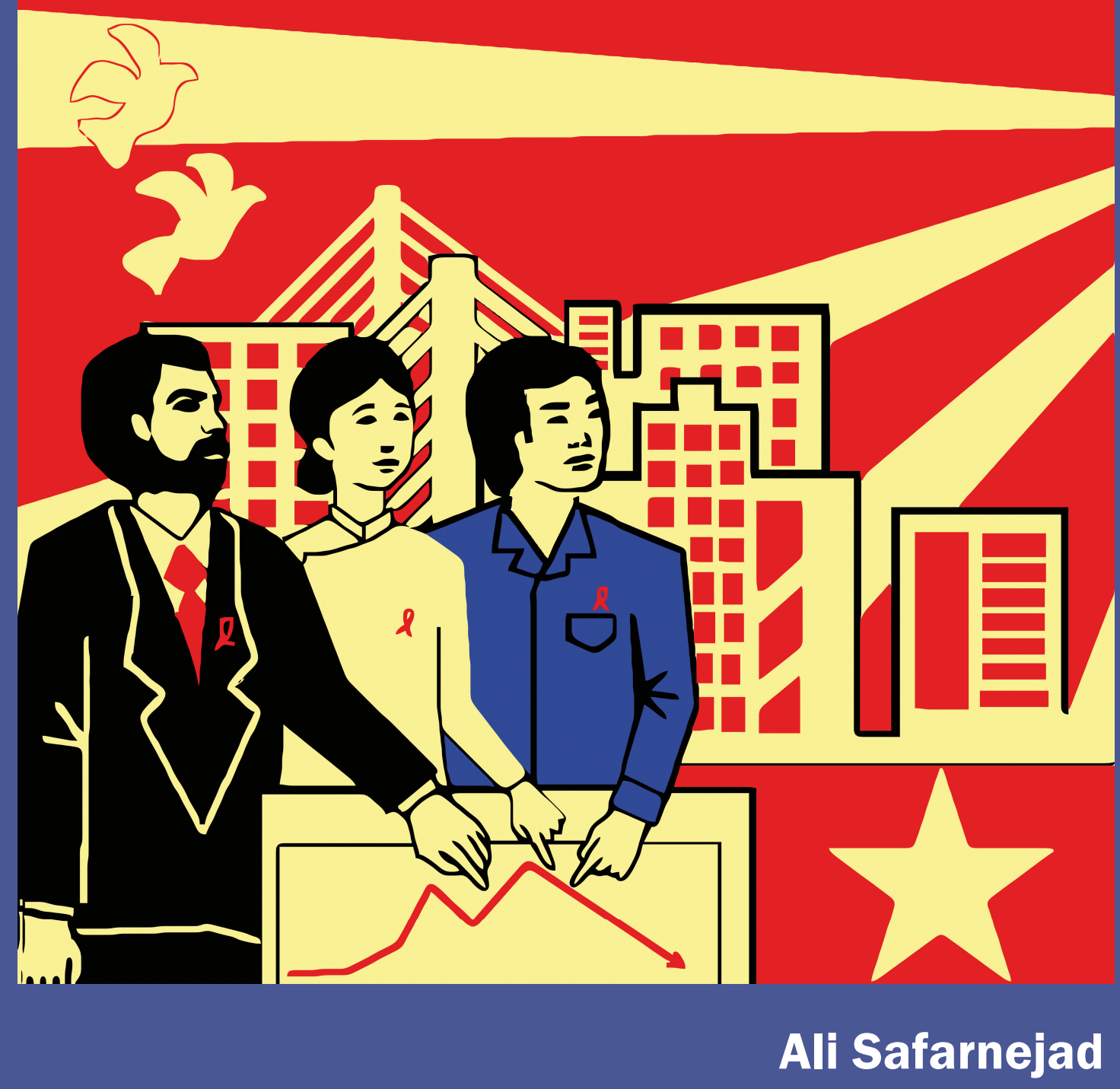




\section{Prioritizing the HIV Response}

A multi-criteria decision analysis

Ali Safarnejad 
(C) Ali Safarnejad, 2017

ISBN: 9789086664344

Published by Boekenplan, Maastricht

www.boekenplan.nl

All rights reserved. No part of this publication may be reproduced, stored in a retrieval system, or transmitted in any form or by any means, electronic, mechanical, photocopying, recording or otherwise, without the written permission from the author. 


\title{
Prioritizing the HIV Response
}

\section{A multi-criteria decision analysis}

\section{DISSERTATION}

\author{
To obtain the degree of Doctor at Maastricht University \\ on the authority of the Rector Magnificus, \\ Prof. Dr. Rianne M. Letschert \\ in accordance with the decision of the Board of Deans, \\ to be defended in public \\ on Thursday, 23 November 2017, at 16:00 hours
}

By

Ali Safarnejad 


\section{Promoter:}

Prof. Dr. Wim Groot

\section{Co-Promoter:}

Dr. Milena Pavlova

\section{Assessment Committee:}

Prof. Dr. Franziska Gassmann (Chair)

Prof. Dr. Rob Baltussen (Radboud University Nijmegen)

Prof. Dr. Silvia Evers

Prof. Dr. Gerjo Kok

Prof. Dr. Hans Maarse 


\section{Table of Contents}

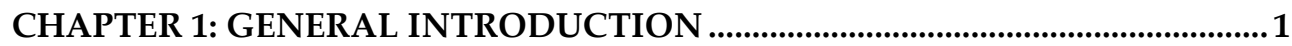

CHAPTER 2: A SYSTEMATIC REVIEW ON PRIORITY SETTING TO HALT AND REVERSE THE HIV EPIDEMIC ...............................................................17

CHAPTER 3: DEVELOPMENT, TESTING AND APPLICATION OF AN INSTRUMENT TO ANALYZE EVIDENCE USE IN NATIONAL STRATEGIC PLANS FOR HIV AND AIDS CONTROL

CHAPTER 4: CHOICES IN SURVEILLANCE OF HIV: LESSONS FROM VIET NAM. .66

CHAPTER 5: CRITERIA FOR PRIORITIZATION OF HIV PROGRAMS IN VIET NAM: A DISCRETE CHOICE EXPERIMENT. 97

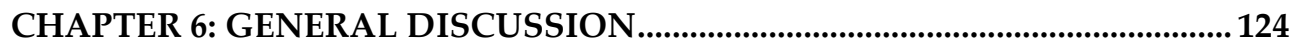

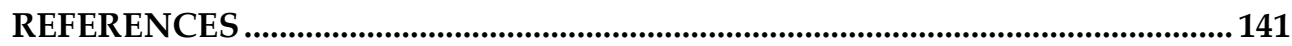

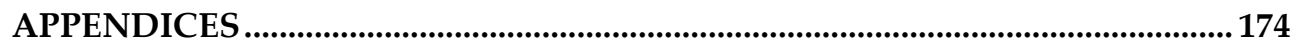

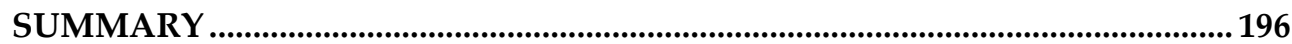

VALORIZATION ADDENDUM....................................................................... 207

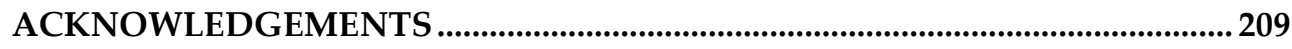

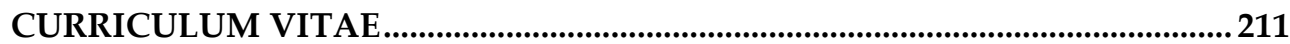




\section{List of Abbreviations}

AEM - AIDS Epidemic Model

AHRQ - Agency for Healthcare Research and Quality

AIDS - Acquired Immune Deficiency Syndrome

CDC - The United States Centers for Disease Control and Prevention

DALY - Disease Adjusted Life Year

DCE - Discrete Choice Experiment

GFATM - The Global Fund to fight AIDS, Tuberculosis and Malaria

GNI - Gross National Income

HIV - Human Immunodeficiency Virus

LGBTQ - Lesbian, Gay, Bisexual, Trans and Queer

LMIC - low- and middle-income countries

MCDA - Multi-Criteria Decision Analysis

MSM - Men who have Sex with Men

NSP - National Strategic Plan

PEPFAR - The U.S. President's Emergency Plan for AIDS Relief

QALY - Quality-Adjusted Life Year

UNAIDS - Joint United Nations Programme on HIV/AIDS

WHO - World Health Organization 
Chapter 1: General Introduction 


\subsection{Focus and scope of the dissertation}

This dissertation focuses on priority setting policy in the response to HIV and AIDS. The demand for healthcare always exceeds its supply, so priority setting is inevitable. Today this focus on priority setting is even more relevant because the aim to end AIDS as a public health threat is faced with the reality of shrinking political and financial resources to end AIDS (Graham-Harrison, 2016; KFF \& UNAIDS, 2016; “Thirty years of a disease: The end of AIDS?," 2011; UNAIDS, 2016d). But above all, with limited resources, a prioritized HIV response is imperative in order to equitably treat the most vulnerable populations at risk of or living with HIV.

Priority setting in global health dates back to the 1980s (Glassman et al., 2012). A number of methods have been developed and attempted since then to aid explicit and rational priority setting (Baltussen \& Niessen, 2006; Kenny \& Joffres, 2008). However, priority setting processes in low- and middle-income countries (LMIC), and also in high-income countries, frequently fail to meet expectations (Glassman et al., 2012; Hipgrave, Alderman, Anderson, \& Soto, 2014; Sabik \& Lie, 2008). Reviews of priority setting point to two factors that contribute to the failure of aided prioritization processes. First, decisions are informed by poor or no evidence on the decision choices and the consequence of those choices (Glassman et al., 2012; Kenny \& Joffres, 2008; Sabik \& Lie, 2008). Second, priority setting processes frequently neglect to involve stakeholders in decision-making (Glassman et al., 2012; Ham, 1997; Kenny \& Joffres, 2008; Sabik \& Lie, 2008). In a review of different methods of priority setting, Multi-Criteria Decision Analysis (MCDA) was proposed as a framework that may work well in LMIC although it requires more experience in its application (Hipgrave et al., 2014). 
This dissertation focuses on the appropriateness and applicability of the MCDA framework to prioritizing the HIV response. It takes this perspective while considering the two previously mentioned problems that afflict prioritization processes: lack of stakeholder involvement and lack of quality evidence for decisions. To apply the MCDA framework and study its applicability, it is necessary to identify relevant criteria for decision-making and to have reliable and good quality evidence (Crown, 2009). So the criteria that are relevant to the HIV response, as well as the state of evidence on HIV, are systematically reviewed in this dissertation.

Although the dissertation takes stock of relevant criteria globally and evaluates the state of evidence for prioritization across countries, actual HIV programs are always implemented at the national or sub-national level. This dissertation therefore focuses on one country - Viet Nam - as a case study of the feasibility of applying MCDA, using country relevant criteria and evidence. As a transitioning economy (World Bank, 2013), and also one designated as a "fast-track" country by major development partners (Emond, 2017), Viet Nam offers an interesting case study due to the challenges it faces in financing the response to a sizable HIV epidemic. Since its graduation to LMIC status, a major challenge for Viet Nam has been the rapid decline in donor funded HIV programs and the subsequent prioritization of programs with available resources. Another challenge has been the inclusion of the growing voices of civil society in the priority setting process.

Findings of this dissertation can be used to inform and guide priority-setting policies in the HIV response, and potentially other communicable diseases faced with scarcity of resources and the imperative of reducing disease burden and risk. Findings of this dissertation also contribute to implementation science in testing the adequacy of a priority setting process in the HIV response. 
The analysis of the decisions or their outcomes is outside of the scope of this dissertation. The analysis of the relevance of different theories of prioritization is also outside of the scope of the dissertation.

The following sections introduce an overview of the history of HIV, discuss in depth the problems of prioritization, review the theoretical origins and debate around prioritization including gaps in research, and introduce the research questions of this dissertation.

\subsection{Background}

In 1982, the United States' Centers for Disease Control and Prevention (CDC) first used the term Acquired Immune Deficiency Syndrome or AIDS, to refer to the condition of diminished resistance to diseases afflicting an increasing number of young, previously healthy gay men, and people who inject drugs (Centers for Disease Control, 1982). By 1983, doctors at the Pasteur Institute in France reported the discovery of the virus, the human immunodeficiency virus or HIV, which could be the cause of AIDS (Barre-Sinoussi et al., 1983). By the end of the millennium, it was estimated that there were 28.9 million people living with HIV in the world (UNAIDS, 2015). Today there are over two million new infections and one million AIDS deaths estimated annually. Around two thirds of these new HIV infections are occurring in Sub-Saharan Africa.

Given the lack of information on HIV in the early years of the epidemic, people living with the disease, or at-risk of infection, created communities to exchange information and test potential treatments (NYPL, 2015). Political response to the HIV epidemic grew out of activism of these communities of people. A number of 
institutions were also established in response to the HIV emergency. The late 1980s saw the genesis of many National AIDS Commissions which were public institutions set up to coordinate the HIV response in a country afflicted by HIV (Spicer et al., 2010). The principle of Three Ones adopted in 2004 reaffirmed the coordination role of the National AIDS Commissions (UNAIDS, 2004a). The Three Ones calls for "one national AIDS coordinating authority", "one agreed HIV/AIDS action framework" and "one agreed monitoring and evaluation system".

The financing of the global HIV response has been unprecedented. Between 1996 and 2003, the global spending on AIDS increased from $\$ 300$ million to $\$ 5$ billion (KFF, 2002) (UNAIDS, 2004c). By 2014, the total resources available for HIV and AIDS in LMIC amounted to $\$ 19$ billion (UNAIDS, 2016b). The United States is the largest bilateral donor to the HIV response, accounting for two-thirds of donor government disbursements, followed by the United Kingdom (13.0\%), France (3.5\%), Germany (2.7\%) and the Netherlands (2.3\%) (KFF \& UNAIDS, 2016).

Despite the achievements made in the past three decades, a massive challenge remains to test and treat the remaining 15 million people estimated to be living with HIV, who are not currently on treatment. Meanwhile, despite the projected need to increase AIDS spending from US\$19 billion available in 2014 to US\$26.2 billion by 2020, donor spending to address HIV in LMIC declined by more than US\$1 billion in 2015 (KFF \& UNAIDS, 2016; UNAIDS, 2016d). This shortfall in funding necessitates priority setting in the national HIV response, particularly in LMIC. Recent evidence suggests that re-evaluation of priority policies may be advisable even among efficient health systems and populations with high levels of health (Defechereux et al., 2012; Koopmanschap, Stolk, \& Koolman, 2010; Mentzakis, Paolucci, \& Rubicko, 2014). 
A motive that dominates priority setting is social justice. Inequities in risk and the burden of disease are reasons in themselves to prioritize certain populations, locations and interventions. For example, it is well known that in all countries, key vulnerable populations are disproportionately affected by HIV (WHO, 2014b). Moreover, evidence shows that people living with or at risk of HIV are at a disadvantage in accessing health and employment due to stigma and discrimination (GNP+, 2016; UNAIDS, 2016e). And HIV is not only driven by the inequalities in health and wealth, but also entrenches them (Cabassi, 2004). The principle of social justice is then an imperative that obligates decision-makers to prioritize the most vulnerable populations.

At the United Nations General Assembly High Level Meeting in June 2016, Member States committed to ending AIDS. A political declaration was adopted with time-bound targets and actions that must be achieved by 2020, including having $90 \%$ of all people living with HIV tested, $90 \%$ of people tested on antiretroviral treatment, and $90 \%$ of people on treatment having suppressed viral loads (UNAIDS, 2016a). Over 200 cities and municipalities across the globe, including Amsterdam, Johannesburg, Mumbai, Kyiv, Mexico City, and Rio de Janeiro, have pledged to achieve the 90-90-90 targets (UNAIDS, 2016c).

Given the aforementioned shortfall in funding, the imperative of social justice, and the urgency of reaching targets to end AIDS, a process of priority setting is needed in all countries with an HIV epidemic.

\subsection{Unsolved HIV priority-setting challenges}

Prioritization decisions allocate funding to programs which are the highest priority, and withdraw funding from programs that are not considered a high 
priority. Policymakers routinely make these types of decisions at some level, either explicitly or implicitly. Good decisions on effective policies and programs require timely, accurate, and relevant information (Stansfield, Walsh, Prata, \& Evans, 2006). Sometimes there is not much information available and decisions become intuitive. A review of health priority setting has found that decisions, particularly in LMIC, frequently rely on poor or no evidence on the choices available and the consequence of those choices (Glassman et al., 2012; Health Metrics Network, 2008; Kenny \& Joffres, 2008; A. R. Ravindran, 2008; Sabik \& Lie, 2008).

A challenge to prioritization is how to meaningfully involve stakeholders in the decision-making process. The principle of greater involvement of people living with or affected by HIV and AIDS was a cornerstone of the Paris AIDS Summit's Declaration in 1994 (Cabassi, 2004). However, translating these commitments into practice is fraught with challenges (Roy \& Cain, 2001). Reviews of health priority setting processes have found that the stakeholder involvement is frequently neglected (Glassman et al., 2012; Ham, 1997; Kenny \& Joffres, 2008; Sabik \& Lie, 2008). When stakeholders are involved in priority setting processes, it is not evident to what extent and at what level they are involved, and whether their participation is meaningful. While the involvement of stakeholders is not a guarantee of improved decisions, their exclusion will most certainly jeopardize the credibility and acceptance of the decisions (Daniels \& Sabin, 2000; Viergever, Olifson, Ghaffar, \& Terry, 2010).

Without a systematic process in place for priority setting, ad hoc decisions will be taken, influenced by heuristics, conventions, intuition and political agendas (Baltussen \& Niessen, 2006; Dean \& Sharfman, 1996; Goddard, Hauck, Preker, \& Smith, 2006; Hunsmann, 2012; Kapiriri \& Martin, 2007; Keeney, 1982; Youngkong, Kapiriri, \& Baltussen, 2009). Following the introduction of the investment 
framework in 2011, countries were given the opportunity to use the investment case approach to prioritize their HIV response (Schwartländer et al., 2011). The investment case is an instrument for strategic decision-making around resource allocation (UNAIDS, 2013b). The investment case approach advances involvement of stakeholders and use of evidence for priority setting. However, the approach misses opportunities in involving stakeholders at critical points in the decisionmaking process, and limits its use of decision criteria to where quantifiable evidence is available. These conditions may lead to inconsistent decisions, which can further exacerbate financial problems, by incurring high and hidden costs (Kahneman, Rosenfield, Gandhi, \& Blaser, 2016).

\subsection{Theoretical origins and debate in priority setting}

Prioritization involves making choices, and choices have consequences. These consequences can be economical, political, social, and in the domain of health, clinical, in nature. Consequently, there are multiple sectorial theories and frameworks that guide decision-making.

The core debate in prioritization in the health sector is around a number of different theories that guide the distribution of health resources from perspectives of utility, cost-effectiveness and equity (Cleary, 2010; Mahapatra, 2002; Mooney, 1987; Newdick, 2005). These theoretical frameworks for rational prioritization can be separated into two camps: the substantive and the procedural solutions (Newdick, 2005; Vlek, 1984). Substantive rationality is exercised when the appropriate solutions have been chosen. Procedural rationality is exercised when appropriate procedures have been taken to choose a solution. Another dimension to substantive or procedural rationality is the values which serve decision-makers in setting priorities (Wenstøp \& Magnus, 2001). Unless specific and enforced rules 
guide the decision-making, it is important for values to be explicit and well founded in a value-focused rational priority setting process. The substantive solutions are essentially guided by cost-effectiveness, where effectiveness is ruled by the number of quality-adjusted life years gained. Procedural prioritization does not have a pre-defined set of criteria, but is rather decided on through a systematic process.

Numerous studies have used the substantive cost-effectiveness paradigm for prioritization. The United Nations Development Programme and the World Bank notably use cost of interventions together with global-burden-of-diseases rankings, a framework widely used to guide health research priorities (Lopez, Mathers, Ezzati, Jamison, \& Murray, 2006), to prioritize the allocation of resources for health interventions (Mahapatra, 2002). There are noted problems with this approach, namely the oversimplification of complex problems into one-dimensional cost comparisons, aggregation of multiple stakeholder interests into a summary effect, and translation of effects at different points in time into a present value calculation (Söderbaum, 2006). Some of these shortcomings have been overcome, like a two stage cost-effectiveness analysis, one for short-term, and another for long-term consequences (Alistar \& Brandeau, 2012). A few cost-effectiveness studies compare effectiveness defined in terms of maximized population health to equitable health in the population (Cleary, 2010; Lasry, Zaric, \& Carter, 2007). McGough et al considered "fair chance" and "best outcome" - variations on equity and maximum health respectively - in setting priorities for rationing antiretroviral treatment to HIV positive patients (McGough, Reynolds, Quinn, \& Zenilman, 2005). However, they note also the limited considerations of criteria of feasibility, long-term effects, and externalities. 
Under conditions of complexity, lack of information, and high uncertainty, substantive rationality gives way to procedural rationality in deciding the best course of action (Vlek, 1984). A number of theorists have operationalized the procedural solution with a finite set of criteria, like Musgrove's nine criteria of public health care spending (Musgrove, 1999). Tromp and Baltussen propose a multi-criteria health priority setting aid that includes 31 criteria under five categories (Tromp \& Baltussen, 2012). Gericke et al identified five criteria to inform priority setting in health (Gericke, Kurowski, Ranson, \& Mills, 2005). Research on deciding on adoption of new vaccines and immunization policy analysis, has resulted in its own set of criteria (Bryson, Duclos, Jolly, \& Bryson, 2010; Burchett, Mounier-Jack, Griffiths, \& Mills, 2012).

The kaleidoscope of priority setting frameworks, and a plethora of priorities advocated by researchers and lobbyists, is problematic particularly in LMIC where institutional mechanisms are not in place to assess priorities. Additionally, there is often no consensus between donors and national policymakers on what should be prioritized, due to the different frameworks used (Glassman et al., 2012). Where frameworks consider multiple criteria, the lack of information, conflicting criteria, and subjective preferences of decision-makers paralyzes the decision-making process and renders the final results inconclusive (Miller, Barrett, \& Henderson, 2006; Xu \& Yang, 2001).

Dean and Sharfman's Strategic Decision-Making Effectiveness Model links decision process with decision effectiveness. The model's constructs are procedural rationality, which is positively related to decision-making effectiveness, and political behavior, which is negatively related to decision-making effectiveness (Dean \& Sharfman, 1996). The multi-criteria analytical framework addresses the two constructs of the strategic decision-making model, political behavior and 
procedural rationality (Crown, 2009). The multi-criteria analytical framework attenuates the effect of political behavior, by exposing the variety of views and the consequences of individual decisions, ultimately leading to greater consensus. The multi-criteria analytical framework also aids procedural rationality, by synthesizing full and relevant information and overcoming the complexity faced by decision-makers in processing an overload of data and criteria in a short time.

This dissertation is framed under the MCDA, because it is an applied approach, which is grounded in decision theory. MCDA models multiple objectives of decision-makers and their value trade-offs so that choices being considered can be compared consistently and transparently (Keeney \& Raiffa, 1993; Peacock, Mitton, Bate, McCoy, \& Donaldson, 2009). The main steps in the MCDA process are (1) exploration and definition of the problem, (2) selection and structuring the criteria, (3) developing alternative options, (3) scoring the alternatives, (4) weighting the criteria, (5) calculating aggregate scores, uncertainty and reporting findings (Marsh et al., 2016). MCDA does not mimic humans' decision-making behavior and instead includes procedures helping people to identify courses of actions in a manner that is analytically robust and consistent in light of the available information and people's preferences.

In the health sector, a multi-criteria approach is seldom used for prioritization, and rarely used in health policy setting, often remaining in the realm of literature or piloting (Glassman et al., 2012; Musgrove, 1999). Figure 1.1 below shows the dimensions and extent of research in criteria-based decision analysis in the broader health arena and specifically in the HIV response. 
Figure 1.1 Extent of research and use of criteria in health and in HIV response for decision analysis

Single/Dual Criteria

Health

HIV/AIDS
Extensive and on-going research and operational use of Global Burden of Disease ${ }^{1}$, and WHO Cost Effective Analysis ${ }^{2}$

Numerous models to prioritize the HIV response such as Investment Framework 5 , $\mathrm{REACH}^{6}, \mathrm{GOAL}^{7}, \mathrm{AVERT}^{8}$
Multiple Criteria

Nine criteria for health spending ${ }^{3}$, WHO $\mathrm{HSPF}^{4}$, MCDA use in UK, Norway, Portugal, Ghana, Nepal, China, Brazil, New Zealand, etc.

An extensive desk review identified only one study where MCDA was applied to prioritize HIV interventions, in Thailand 9

While not an exhaustive catalogue of all research in health priority setting, the figure shows the limited scope and scale of research in use of multi-criteria prioritization of the HIV response.

To date, however, no studies have considered the multiple criteria relevant to stakeholders for prioritization of a comprehensive national HIV response. Research on prioritization and HIV has either focused on a limited set of interventions, like treatment regimens or prevention methods, or not involved all relevant stakeholders in the priority setting process. They have not considered the complex decision situation faced by a group of experts and non-experts in prioritizing a comprehensive package of interventions that go into a national HIV response. The

\footnotetext{
1 (Lopez et al., 2006)

2 (Edejer et al., 2003)

${ }^{3}$ (Musgrove, 1999)

${ }^{4}$ (World Health Organization, 2007)

5 (Schwartländer et al., 2011)

${ }^{6}$ (Alistar, Brandeau, \& Beck, 2013)

7 (Futures Institute, 2013)

8 (Rehle et al., 1998)

${ }^{9}$ (Youngkong et al., 2010)
} 
rigor of methods also varies between studies, and some do not use an evidencebased framework for decision analysis. Therefore, the application of the framework is in the focus of this dissertation.

\subsection{Specific research questions}

The aim of this research is to study the appropriateness and applicability of the MCDA framework, for prioritizing the HIV response in a multi-stakeholder decision-making process. Building the analytical model requires knowing the multiple criteria that are relevant to the HIV response (Crown, 2009). So our first research question is:

Q1: What are the relevant criteria to prioritize the programs, policies, investments, workforce and technologies that are utilized in responding to the HIV epidemic?

The multi-criteria analytical framework also requires quality evidence to support the analysis of the choices and the potential outcomes of those choices (Crown, 2009). As discussed before, the lack of reliable and good quality evidence is one of the problems that afflict priority setting in global health (Crown, 2009; Glassman et al., 2012; Health Metrics Network, 2008; Kenny \& Joffres, 2008; A. R. Ravindran, 2008; Sabik \& Lie, 2008). With a focus on evidence quality in the HIV response, our second research question is:

Q2: To what extent is reliable and good quality evidence available, accessible and used in planning for the HIV response?

Another problem that afflicts priority setting in global health is the lack of stakeholder involvement in decision-making (Glassman et al., 2012; Ham, 1997; 
Kenny \& Joffres, 2008; Sabik \& Lie, 2008), which jeopardizes the credibility and acceptance of the decisions (Daniels \& Sabin, 2000; Viergever et al., 2010). Our third question therefore explores the difference that inclusion of diverse stakeholders makes in decision-making processes:

Q3: What difference does the inclusion of diverse stakeholders make in decisionmaking processes of the HIV response?

After clarifying the criteria and how the criteria should be measured, building a multi-criteria analytical framework for priority setting requires knowing how much importance or weight to put on each criterion (Baltussen \& Niessen, 2006; Defechereux et al., 2012; Mirelman et al., 2012; Tromp, Prawiranegara, Siregar, Sunjaya, \& Baltussen, 2015; Wenstøp \& Magnus, 2001). Given the importance of involvement of key stakeholders living with or at risk of HIV in the decisionmaking processes raised earlier in this chapter, it is important to know how different stakeholders rate the relative importance of criteria by which the HIV response should be prioritized (Baltussen, Stolk, Chisholm, \& Aikins, 2006). The final question of this dissertation is:

Q4: How do different stakeholders rate the relative importance of criteria for prioritizing the HIV response?

This dissertation relies on the participants' perspectives of the priorities in the HIV response to construct a pattern of meaning. In doing so, it uses a mixed method approach, with quantitative and qualitative data used to support, expand upon, and interpret one another in the course of investigating the research questions. With a particular focus on the voices of the most marginalized people - people living with HIV or at higher risk of HIV - and contextualized in the policies and 
political agenda of the times, this dissertation is influenced most by the transformative paradigm of research (Creswell, 2009; Mertens, 2009; Somekh \& Lewin, 2004).

\subsection{Dissertation outline}

Chapter 2 relates to this research question: What are the relevant criteria to prioritize the programs, policies, investments, workforce and technologies that are utilized in responding to the HIV epidemic? It systematically reviews the relevant criteria to prioritize the HIV response reported in the literature. The findings are stratified by a number of dimensions and grouped according to the broader health criteria to articulate the relevant criteria and illustrate the gaps.

Chapter 3 relates to this research question: To what extent is reliable and good quality evidence available, accessible and used in planning for the HIV response? It reviews the quality and extent of evidence used in planning the HIV response. It looks at the national HIV strategic plans of 21 countries over a span of fifteen years, and analyzes the variation in use of evidence in the planning, as well as variation in quality of the evidence.

Chapter 4 relates to this research question: What difference does the inclusion of diverse stakeholders make in decision-making processes of the HIV response? It explores the underlying causes of gaps in evidence, and the value provided by the involvement of stakeholders in the process of priority setting. Through in-depth interviews with health program managers, technical experts, and community members of people at risk of HIV, a picture emerges on the key motivations forand barriers to- efficient generation and use of quality evidence. 
Chapter 5 relates to this research question: How do different stakeholders rate the relative importance of criteria for prioritizing the HIV response? It conducts an experiment to study the preferences and trade-offs made between different HIV programs by multiple stakeholders and decision-makers. The experiment involves participants from government, civil society, and international development partners, and uses modeled scenarios of HIV programs to understand the tradeoffs they make to decide on the preferred program. In doing so, it brings clarity to the importance of criteria used in the HIV response priority setting process to multiple stakeholders.

Chapter 6 draws together the analysis from the previous chapters with five cross cutting key statement. The grounding of these statements are explained, referring to various chapters of the dissertation, followed by recommendations and/or policy implications. The limits in generalization of the statements are also explained in the chapter, as well as future research needs. 


\section{Chapter 2: A Systematic Review on Priority Setting to Halt and Reverse the HIV Epidemic}

This chapter draws upon:

Safarnejad A, Groot W, Pavlova M. A Systematic Review on Priority Setting to Halt and Reverse the HIV Epidemic. Submitted for publication. 


\section{Abstract}

As the 2015 end date of the Millennium Development Goal to halt and reverse the AIDS epidemic nears, ambitious new targets and priorities are being considered. Faced with financial constraints and competing agenda in global health and development, this study explores and articulates the criteria to prioritize the HIV response.

We conducted a systematic review of literature to identify the existing criteria. Cluster analysis was used to classify the criteria into thematic groupings. The criteria were stratified by a number of dimensions and compared by the frequency of their occurrence. HIV/AIDS criteria were also compared with the criteria from the health sector.

Thirty unique criteria were identified in the review, and were clustered into 18 thematic groups. Equity and severity of disease were the most frequently occurring criteria. Two themes around women/mothers and social justice emerged in the analysis. Our review also found that vulnerable and needy populations were more frequently cited as criteria in prioritization of HIV programs than in the broader health sector, illustrating the gap in priorities between the sectors, and underlining the need to consider multi-sectorial criteria in any possible integration of HIV programs into the health systems.

In line with the findings of the study, and recognizing the priorities that contributed to the success of the HIV response, we recommend that the discourse around prevention among high-risk and vulnerable groups, particularly women and girls, be amplified in consideration of policies of prioritization, particularly in concentrated epidemics. 


\subsection{Introduction}

The sixth of eight Millennium Development Goals adopted by world leaders in September of 2000, and reaffirmed in June of 2011, calls for halting and beginning to reverse the spread of HIV by 2015 (UN, 2011). Recently, the idea of the "end of AIDS" has been discussed in the post-2015 MDG context, with an "elimination" vision set for 2030 (Deeks, Lewin, \& Havlir, 2013; UNAIDS, 2010b).

These 2015 goals are estimated to require US\$22-24 billion in investments globally (Schwartländer et al., 2011; UN, 2011), which is a twenty percent increase from the estimated US\$18.9 billion available for HIV programs in 2012 (UNAIDS, 2013a). With new treatment guidelines published by WHO in 2013 recommending earlier initiation of patients on antiretroviral treatment (WHO, 2013a), global costs are expected to be even higher than previously estimated. Meanwhile, donor funding for HIV and AIDS has remained essentially unchanged since 2008 at US\$7.86 billion (The Kaiser Family Foundation \& UNAIDS, 2013), placing the burden of closing the resource gap on domestic sources in low- and middle-income countries.

In terms of global burden of diseases, HIV and AIDS accounted for 3.3 percent of DALYs among 291 causes in 2010 (Christopher J L Murray et al., 2012) and for 2.9 percent of deaths globally, among over 100 causes (WHO, 2011). HIV is only one of the three diseases named in MDG six (besides malaria and tuberculosis) and the three diseases together account for one among the eight goals targeted in the global development agenda.

Given these ambitious targets, cost constraints, and competing agenda in global health and development, a prioritization in allocation of limited resources is needed. In the absence of explicit priority setting, decisions made are those which 
are the least controversial or favor the social, economic and political elite (Ainsworth \& Teokul, 2000; Rosen, Sanne, Collier, \& Simon, 2005a). To be consistent, legitimate, accountable and fair, the priority setting process must have an explicit, exhaustive and deliberate set of criteria (Baltussen \& Niessen, 2006; Bennett \& Chanfreau, 2005; Daniels \& Sabin, 2000).

A number of studies have reviewed the criteria used in health care priority setting (Musgrove, 1999; Tromp \& Baltussen, 2012; Vogel et al., 2013). Most notably, Guindo et al (Guindo et al., 2012) reviewed and identified 58 healthcare decision criteria under nine categories. However, there are no known studies that have systematically reviewed relevant criteria to prioritize the programs, policies, investments, workforce and technologies that are utilized in responding to the epidemic of HIV and AIDS (referred to as "HIV response" hereinafter). As a phenomenon with consequences that reach beyond health, and affects human rights, social justice, stigma, poverty, food security and gender issues among others, HIV is an exceptional disease requiring specialized criteria for priority setting.

This study explores the criteria at play in the HIV response priority setting. The relevant criteria are identified through a systematic review of literature. The identified criteria are mapped to Guindo et al's general healthcare prioritization criteria, and the gaps and overlaps between the two sets are explored. The study also explores the differences in criteria when epidemic type and income levels of countries are considered. Similarities and differences are also explored according to the character (observed/descriptive or recommended/normative), the administrative level (global or national level), and the time period in which the criteria were elicited. 
This is the first known review that uses cluster analysis to classify criteria. It articulates the criteria relevant to the HIV response for use in priority setting and policy making. The criteria elicited in this review are a reflection of how HIV and AIDS are viewed and weighed societally and thus relevant to researchers and policy-makers.

\subsection{Methods}

To identify and describe the criteria used in the HIV response priority setting, a systematic literature review was conducted. The databases PubMed and Science Direct were searched for articles on HIV response priority setting. The search strategy involved an optimization of the search terms followed by searching the databases with those terms.

A first set of search terms relevant to priority setting were extracted from the studies by Guino et al, Tromp \& Baltussen, and Musgrove (Guindo et al., 2012; Musgrove, 1999; Tromp \& Baltussen, 2012). The keywords section, MeSH terms, and the title and abstract of these articles were reviewed for key search terms. These terms were then used together with HIV and AIDS related terms for an initial search in the databases. The resulting articles were scanned and the first five articles that met the inclusion criteria and were relevant to HIV response priority setting were used to extract additional search terms to add to the existing search phrase. Also, the citations in these five relevant articles were scanned, and if any of the cited articles were found relevant, the key terms from those articles were also appended to the search phrase. 


\section{Search Phrase}

"Health care rationing" OR (C"Resource Allocation" OR "Cost-Benefit Analysis" OR

"Decision Making") AND ("priority setting" OR "prioritization" OR "prioritisation" OR

"health priorities")) AND ("HIV" OR "AIDS" OR "Antiretroviral" OR “Anti-Retroviral”)

The search phrase was entered into the databases to identify relevant articles. The abstract and title of the articles were screened to remove articles that did not elicit information about priority setting in the HIV response. The remaining articles were further screened for relevance. Only articles published since 2000, which were dealing with HIV/AIDS criteria for prioritization and considering treatment in their set of interventions, were included in this review. The latter exclusion criterion regarding treatment was added because it is the only intervention currently available to address the goals of eliminating AIDS related deaths. Discarding this criterion would admit an implicit prioritization of at-risk-butuninfected populations over people living with HIV and AIDS.

Additional exclusion criteria were applied to filter relevant articles. Articles dealing with prioritization of health research or health technology, which involved a different set of criteria, were excluded. For example, clinical studies researching criteria for the selection of different drug regimens to administer to patients were excluded. Articles that polled opinions on criteria, or debated the value trade-offs of a fixed set of criteria, or promoted increased attention to a single criterion, were not included in this review. This is because that type of research does not deal with the breadth of criteria involved in decision-making, nor the contextual limitations that determine which criteria can be operationalized. Articles that did not elicit an operational definition of criteria were excluded. For example mentioning equity without discussing the type of equity or how it would be implemented was grounds for exclusion. Language of articles was limited to English. 
The articles were analyzed using thematic content analysis. The articles were read for general impressions. Key terms related to criteria for prioritization were coded inductively, during and after the review of articles. Preset concepts (defined in healthcare criteria set) and emergent themes (defined after reviewing the articles) guided the coding process. Identified codes were then categorized using hierarchical cluster analysis. All cluster analysis was run using the R Statistical Software version 3.0.2 (RCore, 2013). Another categorization of criteria was done by regrouping the codes identified in this systematic review around the themes identified in the systematic review of healthcare priority setting criteria (Guindo et al., 2012). The ranking of criteria themes according to their frequency of occurrence in the HIV and AIDS set and the healthcare set were compared, and thematic gaps between the two set were identified. The final analysis saw the stratification of articles by administrative level, the income group of countries under study, type of the epidemic, whether the article was characterized as normative or descriptive, and the year of publication of the article.

\subsection{Results}

The literature search resulted in a total of 840 records identified through searching PubMed and Science Direct databases. These records were screened by their title and abstracts and 649 were excluded. The remaining 95 were assessed for eligibility, on the basis of their full text, and 66 were excluded. A total of 29 publications that met the limitations and relevance criteria were included in the literature review (Figure 2.1). The results of the review are presented in this section. 
Figure 2.1 PRISMA diagram

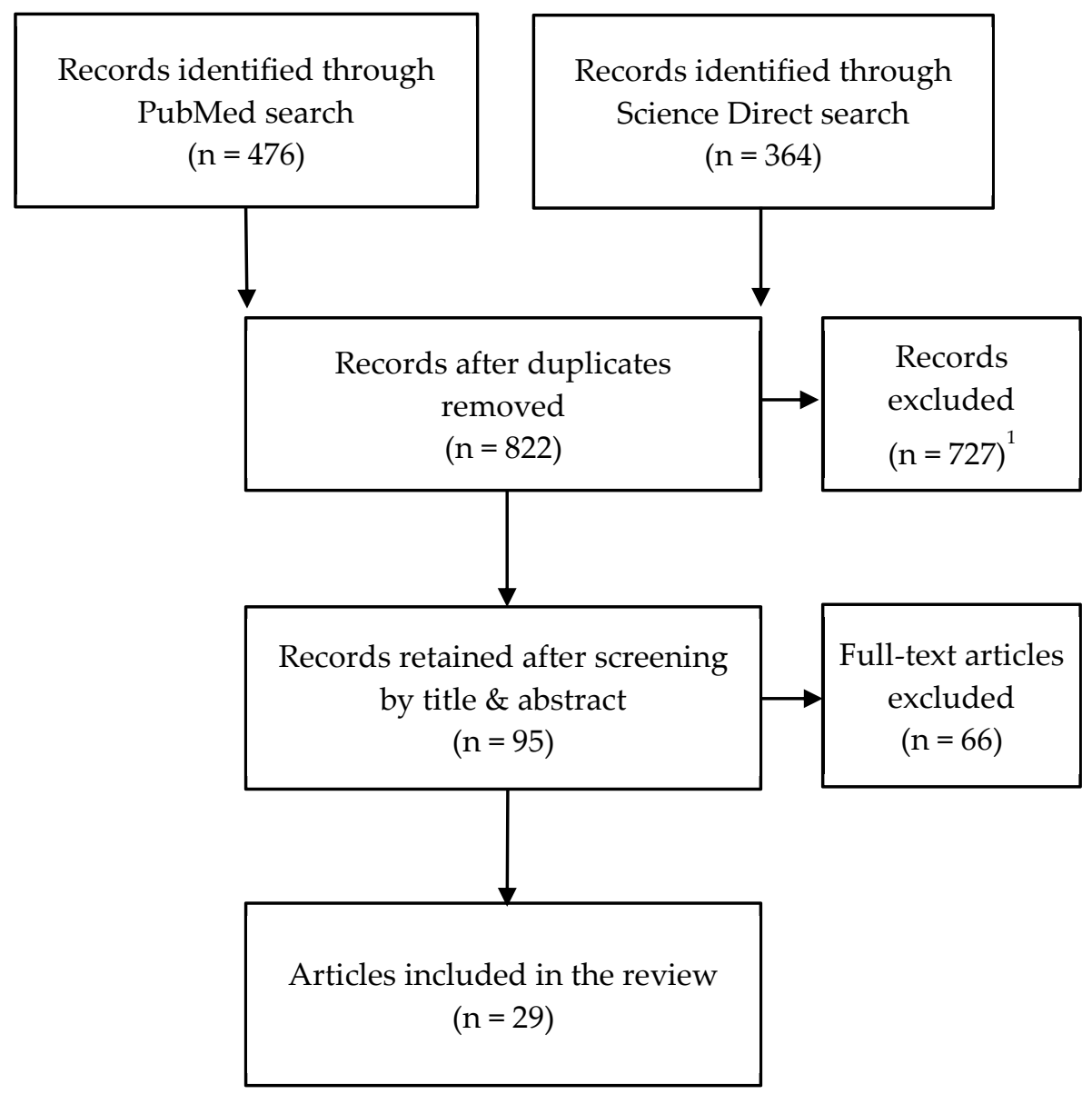

Note: ${ }^{1}$ Of 727 excluded records, 649 did not pass screening, 65 were not found, and 13 were duplicates.

\section{Descriptive characteristics of the included articles}

The 29 articles included in the review were published in 20 different peer-reviewed journals (Appendix A). They represent studies from 14 different countries, ten of which are from Sub-Saharan Africa. Most articles were published in journals with 
impact factors of five or less, although six articles were published in journals with a high impact factor $(>15)$. There is a constant increase over the years in the number of publications eliciting HIV and AIDS prioritization criteria with a peak in 2005 . Nearly half of the articles (14/29) are editorials, appearing in commentary, viewpoint, and policy review sections of the journal. The remaining articles are qualitative, quantitative or mixed-method design studies. The majority of the articles (24/29) focus on which populations to prioritize, and the remainder either focus on criteria to prioritize interventions or both population and intervention.

\section{Criteria and frequency of occurrence}

During the review, 30 different terms were identified eliciting criteria used in priority setting. Some of the terms contain sub-terms that operationally define the criteria. For example, equity is defined by sub-terms of gender equity, geographical equity, racial equity, and economic equity. Other criteria have distinct and nonoverlapping sub-categories like age, disaggregated as children and adults. Where multiple definitions of the same concept have been elicited in the reviewed articles, they have been aggregated into a single criterion. Cost-effectiveness for example can be contextually measured as "cost per infection averted", or "cost per DALY which is less than GNI per capita", or "cost per QALY".

Frequency counting was used to rank order the 30 unique criteria. The top criteria, occurring in at least one-third of the publications, are: 1) equity, 2) severity of disease, 3) human capital, 4) feasibility, 5) adherence (psychological and structural), 6) age, g) affordability 7) fairness 8) pregnant women. The unique criteria and proportion of publications that elicited them are presented in Table 2.1. 
Table 2.1 HIV/AIDS related criteria elicited in reviewed articles and frequency of their occurrence

\begin{tabular}{ll}
\hline Criteria & Frequency of \\
& Occurrence (\%) \\
\hline Equity & 79.3 \\
Severity of disease & 72.4 \\
Human capital & 44.8 \\
Feasibility & 44.8 \\
Adherence - Psychological & 41.4 \\
Adherence - Structural & 41.4 \\
Age & 41.4 \\
Affordability & 37.9 \\
Fairness & 34.5 \\
Pregnant women & 34.5 \\
Gender & 31.0 \\
Sustainability & 27.6 \\
Stigma and Discrimination & 27.6 \\
Coverage & 24.1 \\
Equal Worth of Life & 24.1 \\
High-risk Population & 20.7 \\
Accessibility & 20.7 \\
Cost-Effectiveness & 17.2 \\
Efficacy & 17.2 \\
Access Selection Committee & 17.2 \\
Health Outcomes & 17.2 \\
Women of Childbearing Age & 13.8 \\
Mothers & 13.8 \\
Parents & 13.8 \\
Profession - Unemployed & 13.8 \\
Innocent Victim & 10.3 \\
Epidemiological/Prevention - At Risk Populations & 3.4 \\
Orphans/Widows & 3.4 \\
Quality of Care & 3.4 \\
Safety of Measure & 3.4 \\
\hline & \\
\hline
\end{tabular}




\section{Thematic grouping of criteria}

To explore the possible thematic groupings, cluster analysis was conducted on the criteria. The analysis involved converting the occurrence of criteria into numeric data, and applying hierarchical clustering to explore how the criteria grouped together. The cluster analysis produced 18 thematic groups from the set of 30 criteria. For presentation purposes, criteria that occurred in three or fewer articles, and did not thematically group together with other criteria in a meaningful way, were removed from the dendrogram (Figure 2.2).

Table 2.2 HIV/AIDS related criteria themes developed following hierarchical clustering

\begin{tabular}{ll}
\hline Criteria & Frequency of \\
& Occurrence (\%) \\
\hline Equity & 79.3 \\
Severity of Disease & 72.4 \\
Economics & 48.3 \\
Social Justice & 44.8 \\
Feasibility & 44.8 \\
Adherence - Psychological & 41.4 \\
Adherence - Structural & 41.4 \\
Age & 41.4 \\
Affordability & 37.9 \\
Women / Mothers & 37.9 \\
Fairness & 34.5 \\
Gender & 31.0 \\
Population Effects / Prevention & 31.0 \\
Coverage & 24.1 \\
Access Selection Committee & 17.2 \\
Cost-Effectiveness & 17.2 \\
Efficacy & 17.2 \\
Unemployed & 13.8 \\
\hline
\end{tabular}


Figure 2.2 Cluster dendrogram exhibiting grouping of criteria

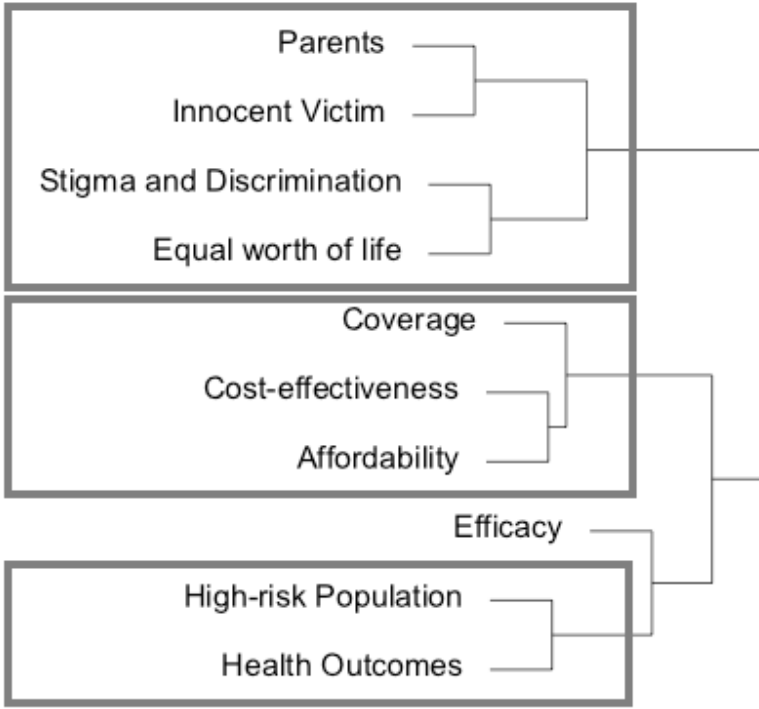

Adherence - Psychological
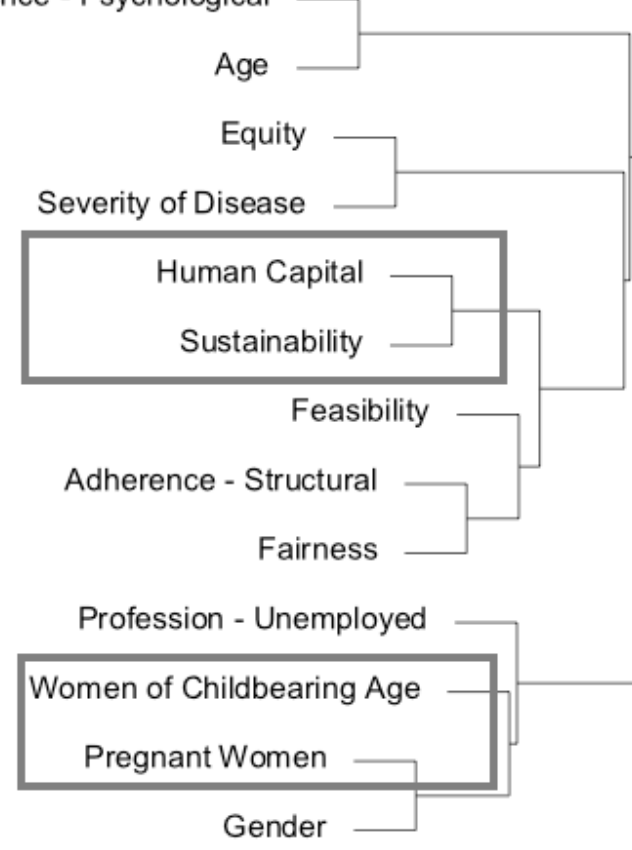

Access Selection Committee

Accessibility

Mothers 
The hierarchical cluster analysis shows a grouping of social justice concepts, including parents, innocent victims of rape or abuse, stigma and discrimination, and equal worth of life. Prevention efforts focused on high-risk populations has upstream effects benefiting population health outcomes, thus linking these two concepts under the theme of population effects/prevention. Sustainability by means of co-payments or public funding, and human capital preservation are grouped under an economics theme. Several criteria related to women of childbearing age, pregnant women, and mothers, with focus on prevention of transmission, prevention of orphans, ease of access, and the social good of keeping mothers alive seem to co-occur. These criteria are grouped under the theme titled women/mothers. Once criteria were grouped around the themes developed by cluster analysis, the themes were rank ordered by the frequency of occurrence of their associated criteria (Table 2.2).

\section{Mapping of HIV/AIDS criteria to healthcare decision-making criteria}

Criteria identified through the systematic review were compared to the healthcare decision-making criteria presented by Guindo et al (Guindo et al., 2012). All 30 criteria in the HIV/AIDS set were mapped to the criteria and classifications in the healthcare set. The mapping resulted in 17 themes to be developed that were common to both HIV/AIDS and the healthcare set. Two additional criteria - gender and age (specifically adults) - found no occurrence in the healthcare set (Table 2.3).

The most frequently occurring themes in the common set were vulnerable and needy population, disease severity, equity/fairness/justice, and efficacy/effectiveness, all occurring in more than half of the articles reviewed in this study. The latter two are also the most frequently occurring criteria in the healthcare set. However, the first two occur in only about 22 percent of healthcare set articles. In fact, the greatest 
discrepancy between the HIV/AIDS sets and the healthcare set occurs over these two criteria of disease severity and vulnerable and needy populations, with nearly a 50 percent point difference between the two sets.

Table 2.3 HIV/AIDS related criteria grouped by healthcare classifications

\begin{tabular}{lll}
\hline & \multicolumn{2}{c}{ Frequency of Occurrence (\%) } \\
\hline Criteria & in HIV/AIDS & in Healthcare \\
& Set (\%) & Set (\%) \\
\hline Vulnerable and Needy Population & 75.9 & 22.5 \\
Disease Severity & 72.4 & 22.5 \\
Equity, Fairness, and Justice & 72.4 & 42.5 \\
Efficacy/Effectiveness & 69.0 & 52.5 \\
Economic Impact ${ }^{1}$ & 44.8 & 22.5 \\
Org. Requirements \& Capacity to Implement & 44.8 & 32.5 \\
Access & 41.4 & 35.0 \\
Budget Impact & 37.9 & 22.5 \\
Gender & 31.0 & 0.0 \\
Sustainability & 31.0 & 10.0 \\
Epidemiology & 27.6 & 32.5 \\
Cultural Aspects & 27.6 & 15.0 \\
Population Effect & 24.1 & 17.5 \\
Age - Adult & 20.7 & 0.0 \\
Health Benefits & 17.2 & 22.5 \\
Cost-Effectiveness & 17.2 & 42.5 \\
Stakeholders Interest and Pressure & 17.2 & 30.0 \\
Safety & 3.4 & 35.0 \\
Quality of Care & 3.4 & 2.5 \\
\hline Note: ${ }^{\text {Indudes }}$ & 2.0 & Cost
\end{tabular}

Note: ${ }^{1}$ includes Financial Impact, Poverty Reduction and Opportunity Cost

Certain criteria from the healthcare set did not successfully map to the HIV/AIDS set. For example, patient reported outcomes, individual effects, need, clinical guidelines and practices, pre-existing use, cost, value, resources, insurance premiums, quality of evidence, legislation, skills, flexibility of intervention, characteristics of intervention, appropriate use, barriers and acceptability, 
integration and system efficiencies, population priorities, utility, solidarity, ethics and moral aspects, as well as the overall context criteria, did not appear in the articles selected for this review.

\section{Stratification of criteria}

Equity took up the top ranking position in every mode of stratification of the articles in this review. Severity of disease shared the top ranking position in both strata when articles were stratified by administrative level, by their normative/descriptive character, and by their date of publication. When articles were stratified by income level of the country being studied, severity of disease was the top ranked criterion only among the upper income country set. And when stratified by the epidemic type of the country being studied, severity of disease was the top ranked criterion only among the concentrated epidemic country set.

Stratification by administrative level found 17 articles to have a national or subnational focus, and 12 articles focused globally or regionally. Only three criteria differed in their ranking by 50 percent or more between the two strata: Health outcomes, adherence (psychological), and age. Health outcomes were only a concern at the global level, with no articles in the national set considering it as a criterion. Only two articles out of twelve with a global focus cited adherence and age as criteria, whereas approximately 60 percent of national set articles cited these as criteria. Feasibility was the second highest ranked criteria in the global set, on par with equity. However, in the national set, feasibility was ranked at the median of the criteria. Human capital, the third ranking criteria in the global set of articles, was also lower ranked in the national set, appearing at the fifth of eleven ranking positions (Appendix B). 
Overall, stratification of the articles by their characterization as normative or descriptive did not show significant differences between the two sets. Nevertheless, four criteria showed a 50 percent or higher variations in their ranking between the two sets. Adherence (psychological) ranks third in the descriptive set, but appears infrequently in the normative set. Health outcomes, feasibility, and efficacy all appeared frequently in the normative set, but infrequently in the descriptive set (Appendix C).

Articles with a national level focus were stratified by the gross national income (GNI) per capita of the country being studied. The articles were divided into an upper income set and a lower income set at the median GNI per capita value of $\$ 1,380$. Six criteria showed a 50 percent or more difference in ranking between the two sets. Four of those criteria, adherence (psychological and structural), human capital, and sustainability, were ranked higher in the lower income set, whereas affordability and cost-effectiveness were ranked higher in the upper income set (Appendix D).

Stratification of the articles by the type of epidemic showed significant differences in ranking of five criteria. Adherence (both psychological and structural), fairness, pregnant women, and efficacy all show a difference of 50 percent or more in ranking between concentrated and generalized epidemics. All these criteria were more frequently elicited in the generalized epidemic set than the concentrated epidemic set, except for the efficacy criterion, which never occurs in the generalized epidemic set. Both sets rank high-risk populations in the lower half of their criteria list (Appendix E).

There is only one significant difference between the set of criteria before and after the year 2007. The criterion feasibility appears in over 70 percent of articles from 2000 to 2007. But from 2007 onward, only 20 percent of articles elicit this criterion. 
All other criteria have less than a fifty percent difference in ranking when stratified by period of publication of articles in which they are elicited (Appendix F).

\subsection{Discussion}

This study set out to explore the criteria relevant to the prioritization of the HIV response. It also compared the criteria specific to the HIV response to the broader health sector criteria. The criteria found are impressive in quantity and diversity, spanning issues of public health, social justice, and economic efficiency. Equity and severity of disease were the most frequently cited criteria for prioritization. Stratification by the administrative level of focus of articles, normative or descriptive nature of articles, year of publication of articles, and country income level provided further evidence that equity and severity of disease are the most important criteria at global and national level, in use and in normative guidance, over the past 14 years.

Two important themes were developed in the analysis of the HIV/AIDS criteria: women/mothers and social justice. These two criteria themes are unique to the HIV response in that they underline the feminization of HIV and AIDS, and signify the ethical imperative of prioritizing the most marginalized populations despite higher costs per life-year saved (Moatti, Marlink, Luchini, \& Kazatchkine, 2008; Rosen, Sanne, Collier, \& Simon, 2005b). Indeed, the initial slow response to the epidemic can be attributed to the stigma suffered by the socially marginalized and economically disadvantaged, who were the predominant victims of the disease (Michaelis, 2002). Gender mainstreaming, and particularly a focus on women and girls, has been historically lacking in the objectives or strategies of health programs (Briones-Vozmediano, Vives-Cases, \& Peiro-Perez, 2012; T. S. Ravindran \& Kelkar- 
Khambete, 2007). In contrast, the HIV response has recognized the dynamics between genders that act as barriers to women's access to treatment and care (Dixon, Gibson, McPake, \& Maleta, 2011; Metz, 2008), and prioritized populations based on gender for a number of years (Johansson, Jerene, \& Norheim, 2008; Richter, Hicks, Earnshaw, \& Honeycutt, 2008).

There are clear gaps between the health sector and HIV response, in terms of criteria for prioritization, which has implications for both improving the HIV response priority setting, but also for any consideration of integration of treatment and prevention of HIV and AIDS into the health systems like non-communicable diseases or sexual and reproductive health (Sidibé, Piot, \& Dybul, 2012). The widest gap in criteria for prioritization occurs around the consideration of severity of disease and vulnerable and needy populations, which are frequently elicited in the HIV response, but less frequently in the broader health sector.

Demand for prevention, treatment and care outpacing resource availability has meant prioritizing those in urgent need (Daniels, 2004), hence the frequent reference to severity of disease criterion in the HIV/AIDS set. In fact, the most commonly used criteria for rationing anti-retroviral treatment in the past decade has been the WHO guidelines on CD4 cell count, itself a measure of severity of disease (WHO, 2013a). HIV also affects the wellbeing of those infected, and in a vicious cycle the most vulnerable populations, including women, young people, and marginalized groups, are most at risk of infection (Bradshaw et al., 2008). Criteria that prioritize the vulnerable and needy populations are therefore given a more prominent focus in the HIV response, as reflected in the frequent reference to the criteria that prioritizes this group. 
These two criteria perhaps best illustrate the success of the HIV response in framing the public health and emergency response approach to the epidemic, within the context of social justice and development. Emulation of the successes and lessons from the HIV response, including the criteria used for priority setting, can only serve to strengthen the health system, as synergies with the health sector are pursued. Consideration of policies and practice of integration must be accountable to the key criteria of the HIV response.

Stratification of the articles by their characterization as normative or descriptive shows that feasibility appears infrequently in the descriptive set, although it is the second most important criteria in the normative set. This difference in ranking may be due to a lack of consideration of resource needs in allocation models of the HIV response (Alistar \& Brandeau, 2012; Hunsmann, 2012; Kumaranayake \& Watts, 2001). The gap between the normative and descriptive ranking of the feasibility criterion further stresses the work remaining in operationalizing certain criteria deemed important to an effective response.

Observations from the stratification of criteria by the administrative level of their elicitation suggest that national and sub-national criteria are more prone to have an individual patient level of prioritization (e.g. psychological adherence, age) whereas the global and regional set criteria focus more on societal level priorities (e.g. human capital, health outcomes). In essence, this is a dilemma of public health ethics versus ethics of individual patient care that manifests itself in priority setting (Benatar, 2006).

Assuming that access to treatment and care is more scarce in lower income countries than upper income countries, it can be inferred that lower income countries would prioritize patients who benefit society, consider human capital 
preservation, and limit treatment to those who are most likely to succeed on treatment by adherence to medication. This rational is corroborated by the findings of this study. The findings also show that criteria of affordability and costeffectiveness are ranked higher in the upper income set, suggesting that those countries with fewer resource constraints are more concerned with optimization of the resource allocation to maximize the benefit to cost ratio.

A notable difference when stratifying articles by the income group of the country under study is over the criteria of stigma and discrimination. These criteria are not mentioned in the articles focused on the upper income countries, whereas over one third of the articles in the lower income group elicit the criteria. Factors of education, health literacy, and negative attitudes towards high-risk groups are frequently linked to socio-economics status (Lim et al., 2013; Parker \& Aggleton, 2003; Waite, Paasche-Orlow, Rintamaki, Davis, \& Wolf, 2008). A plausible explanation for the frequent reference to stigma and discrimination in the lower income country strata may be the low knowledge on modes of transmission and consequent negative attitudes toward people living with or at higher risk of HIV and AIDS.

An important finding from the stratification of criteria by epidemic type is the ranking of the criterion on prioritization of high-risk populations. While this criterion should hypothetically weigh more in concentrated epidemics, which by definition are concentrated in key high-risk populations, the generalized epidemic set shows only a slightly lower ranking of this criterion than the concentrated epidemic set. Moreover, high-risk populations ranks in the lower half of all criteria, irrespective of stratification of the criteria in this study, despite this population being a key driver of the epidemic, and most-at-risk of being infected. 
The growing body of literature eliciting criteria for prioritization demonstrates the increased interest in explicit consideration of multiple criteria in health policy decisions. Publications eliciting criteria peaked in 2005, which may be due to the increased focus on priority setting at the end of the WHO 3-by-5 initiative (World Health Organization, 2003). Among the criteria elicited, the only significant change before and after 2007 was around the criteria of feasibility. Frequently cited before 2007, references to feasibility have diminished over time. The dominance of feasibility in the pre-2007 era can be attributed to the weak information and illdefined measure of this criterion (Baltussen et al., 2013; Defechereux et al., 2012; Mahapatra, 2002), which makes its use favorable in reason-based, political decision-making processes (Baltussen \& Niessen, 2006; Keren \& Bruin, 2003). The gradual movement toward evidenced based programming, aided by improved monitoring of the HIV response, vis-à-vis a persistent deficiency in quantitative measurements of feasibility, can be related to the waning elicitation of this criterion in priority setting in recent years. Wider availability of pharmaceutical treatments and prevention interventions provides another possible explanation for the declining emphasis on feasibility over the years.

Cost-effectiveness is a salient strategy for prioritization in the HIV response among major donors and multilaterals (GFATM, 2012; Hogan, 2005; PEPFAR, 2008; The World Bank, 2011). The most widely used tool currently available for national and global HIV response planning is the GOALS model, which optimizes on cost and epidemic effect (Alistar \& Brandeau, 2012; Forsythe, Stover, \& Bollinger, 2009). Cost-effectiveness is also the fourth most frequently reported criterion in health prioritization (Guindo et al., 2012). Nevertheless, findings from this study show that cost-effectiveness is an infrequently cited criterion in the HIV response priority setting overall. At every administrative level, in concentrated and generalized epidemics, among normative and descriptive articles, over the past 14 years, cost- 
effectiveness as a criterion consistently ranks in the lower half of all criteria considered. Only among high-income countries does this criterion get frequently mentioned. This linkage may be attributed to the weak health information system in low- and middle-income countries, where the quality information needed to make informed, reliable decisions on the most cost-effective strategy is unavailable (Health Metrics Network, 2008). Otherwise use of cost-effectiveness as a criterion for priority setting at the global level by donors and multilaterals does not generalize well to its promotion and application in the national context.

This study has required lifesaving treatment to be included in the package of interventions of the HIV response. Although there is a tendency for reviewed criteria in this study to consider how treatment can be rationed, prevention is not excluded from the review. Moreover, an answer to rationing of treatment would also answer the analogous question about how to ration preventative measures (Metz, 2008).

The grouping of criteria was approached in this study with themes identified by existing frameworks and classifications, and followed by applying hierarchical cluster analysis to explore membership of criteria in any new groups. An alternative approach would have been to start with the quantitative cluster analysis on the disaggregated criteria and then qualitatively explore the mapping of the identified themes to existing frameworks and classifications. However, given the large number of criteria in the disaggregated set, to maintain an adequate degree of freedom in the analysis, this latter approach would have required a much larger set of cases from which criteria can be drawn than the present study had available. 


\subsection{Conclusion}

This chapter synthesizes the literature on HIV response priority setting, taking stock of criteria used and articulating discrepancies with criteria from the broader health sector. The review identified 30 unique criteria fitting into 18 categories. These categories can be used in future studies and policy reviews not only to identify which criteria are particularly relevant to national HIV program priority setting, but also to determine conceptual conflicts that exist between pairs of criteria.

Three important findings emerged in the present review. The first concerns the frequent elicitation of equity and severity of disease as important criteria to consider in priority setting in the HIV response. A second area concerns the clustering of criteria around themes of social justice and women/mothers. The third finding emerged in the categorization of criteria around healthcare categories where vulnerable and needy population ranked as the top criterion. These findings together form a societal perspective of HIV as a severe disease that disproportionately affects vulnerable groups, particularly women and girls. Recognizing the priorities that contributed to the success of the HIV response thus far while acknowledging the work remaining ahead, it is crucial then for the prioritization of the HIV response in the post-2015 era to be as much grounded in social justice as it is in optimum public health outcomes.

The review also noted the scarce elicitation of cost-effectiveness and high-risk populations as criteria, in low- and middle-income countries and concentrated epidemics respectively. Weak health information systems and stigma for at-risk groups present some possible barriers to effective application of the criteria in resource poor settings. There is a need for more research exploring other barriers 
that prevent these criteria from being considered in different contexts. Only with better information, explicit criteria, and rational decision-making processes, can the priorities of the HIV response be optimized to reach the vision of elimination. 


\section{Chapter 3: Development, Testing and Application of an Instrument to Analyze Evidence Use in National Strategic Plans for HIV and AIDS Control}

This chapter draws upon:

Safarnejad A, Groot W, Pavlova M. Development, Testing and Application of an Instrument to Analyze Evidence Use in National Strategic Plans for HIV and AIDS Control. Submitted for publication. 


\section{Abstract}

National Strategic Plans (NSP) guide the HIV response in countries and help planners make informed decisions to change the epidemic's trajectory. Given the importance of evidence for decision-making, this study explores the extent of data use in the NSP.

An instrument was developed to measure evidence use in NSP. The internal reliability and external validity of the instrument were tested. NSP's of 21 countries in Africa were assessed with the instrument.

Our results show increasing evidence use over the years, which is consistent with studies that suggest increasing investments on monitoring and evaluation are paying off in terms of improved generation of evidence. Data on epidemic drivers were the most frequently used. Expenditure data and impact/outcome evaluation were the least frequently used data type for planning. Evidence on key populations at risk was largely absent.

Our instrument provides an objective analysis of evidence-based planning in the HIV response. Our results suggest that with regard to the NSP, the generated evidence is being used in planning, and that evidence use is increasing over the years. 


\subsection{Introduction}

Evidence helps to rationalize and improve the quality of decisions. Evidence influences policy and planning by facilitating the recognition of problems, rational decision-making, monitoring, evaluation and forecasting (Adrien et al., 2008; Haskins \& Baron, 2011; Rivlin, 1971). Evidence-based health policies guide and justify actions that are linked to improved health outcomes (Brownson, Chriqui, \& Stamatakis, 2009; The World Bank, 2007; WHO, 2013b). Hence, health information forms one of the six pillars of a strong national health system (Health Metrics Network, 2008).

Given the importance of evidence to improve health outcomes, the WHO Framework for National Health Policies, Strategies and Plans (WHO, 2010) calls for a robust situation analysis as an input to the development of national health policies. Also, a policy tool is made available for the joint assessment of national health strategies and plans (known as the JANS tool). The tool is used to support the review of national health plans (IHP+, 2013).

Similar to the national health plans, National Strategic Plans (NSP) on HIV and AIDS outline the vision for the national HIV response, the desired results, and the approach to achieving them. As early as 1998, the United Nations guided the development of NSP on HIV and AIDS to help planners make informed decisions to improve the epidemic situation (UNAIDS, 1998). The concept of a single action plan that coordinates the work of all partners in the HIV response, was formally endorsed by major donors, international organizations, and national governments in 2004 (UNAIDS, 2004b, 2004d). NSP on HIV and AIDS typically cover a period of several years and involve many areas of development. These plans are used to attract and sustain funding from the national budget and external donors. 
Therefore, NSP on HIV and AIDS need to make careful prioritization, be evidencedriven, and use many different information sources.

A look into the planning process for development of NSP illustrates how inclusion of evidence in the NSP is related to discussion of the evidence in the planning process. Planning for development of the NSP starts with an assessment and analysis of the country situation and response (UNAIDS, 1998; UNDG, 2003). During the analysis phase sources are identified and relevant documents are collected and analyzed, and reports are produced and circulated for comments. The situation analysis will have laid the groundwork for the NSP, with a continuation of working programs in the new plan, and exclusion of initiatives that are irrelevant or not working. The final draft of the strategic plan is circulated widely and finalized following a consultation workshop with all major interest groups involved in the HIV response. The entire planning process is also documented in the final strategic plan.

While several instruments have been developed to assess national health plans (Ciliska, Thomas, \& Buffett, 2012; IHP+, 2013; WHO, 2010), there are no validated instruments for the evaluation of NSP on HIV and AIDS. As HIV and AIDS is a multi-sectorial program in nature, involving different planners, and following different conventions and frameworks than other health programs, an assessment of evidence in NSP on HIV and AIDS also requires a tailored instrument. The aim of this chapter is to develop an instrument that is specific to the evaluation of NSP on HIV and AIDS. We also aim to validate the instrument and use the instrument to assess quality of evidence used in NSP, using information on Sub-Saharan Africa over the period 1998-2014. The process of instrument development includes mapping of a number of existing assessment tools, reconciliation, pre-testing, and instrument adjustments. The instrument validation process involves the 
exploration of reliability, internal consistency, construct validity, responsiveness, and external consistency.

The instrument developed in this chapter applies content analysis methods to review of NSP. Content analysis is a qualitative method of research to analyze text data. This method examines the characteristics of the language in documents to classifying text into categories of similar meaning (USGAO, 1996; Weber, 1990). Content analysis of the NSP provides basic insights on use of quality evidence, but there is no broader analysis of the context of the NSP.

The instrument developed in this chapter measures the use of evidence in policy planning and programming. Measures based on this instrument can be important for donors like The Global Fund to fight AIDS, Tuberculosis and Malaria and other organizations who base their funding on performance. Such measures can help them to justify the rationality of their decisions for funding a given program (Alfven et al., 2014; Peersman, Rugg, Erkkola, Kiwango, \& Yang, 2009). The measures can also be valuable for the countries included in the study that want to address their evidence gaps. And the instrument can be useful for other countries confronted with an HIV epidemic in reviewing their NSP.

\subsection{Methods}

\section{Instrument development}

Since no appraisal instrument exists to score and rank NSP on HIV and AIDS based on the quality and use of evidence, we developed such an instrument. Our study draws on the Handbook on Planning and Managing for HIV/AIDS Results (The World Bank, 2007), and the Guide to the Strategic Planning Process for a 
National Response to HIV/AIDS (UNAIDS, 1998). This handbook was used to select domains of evidence and divide them into categories of evidence. Additional domains and categories were identified based on the Joint Assessment of National Health Strategies Tool and Guidelines (IHP+, 2013), the Compendium of Critical Appraisal Tools for Public Health Practice (Ciliska et al., 2012), and the WHO Framework for National Health Policies, Strategies and Plans (WHO, 2010).

The desk review mentioned above identified independent domains of evidence for the draft appraisal instrument. Each domain of evidence had a number of categories and its own scale for rating the NSP. Measurement of evidence quality was operationalized by the extent to which these categories were present in the NSP. The sum of the category ratings across all domains of evidence in the appraisal instrument operationally defined the use of evidence in the NSP. For each domain, the NSP were rated on the domain-related scale. For this purpose, specific search terms were created to identify domains of evidence in the NSP. For example, search terms to identify evidence under the domain of Program Data included "coverage", "reach", and "distribution". The scales of the domains for scoring the NSP range from zero (indicating no evidence in that domain) to an integer between one and six (indicating the presence of one or more independent and measurable categories of evidence in the given domain). This range is the aforementioned operational measurement of the quality of evidence used in each domain of evidence.

In several domains of evidence a distinction is made between "qualified" evidence and providing the evidence itself. Operationally, "qualified" evidence means the NSP talked about levels or trends of data in relative terms, without giving numerical data values. For example, if an NSP stated that funding for HIV was "insufficient" without stating how much funding was available, the NSP was rated 
as having "qualified" indication of funding. Similarly, if an NSP stated that new HIV infections were "increasing", without giving the HIV incidence rate, that NSP would be rated as having "qualified" trend in epidemiological data. By giving valued measurements a higher score than qualified evidence, we operationalized the quality of evidence use in NSP in relevant domains of evidence.

The draft appraisal instrument was piloted on five NSP on HIV and AIDS. These NSP were randomly selected from among the 27 NSP that would be evaluated in the study. The one rater who carried out the pilot evaluation of five NSP was the same person who would evaluate the full set of 27 NSP. Piloting of the instrument identified new domain categories which were added to the appraisal instrument. When categories reached saturation - defined as no new categories identified in two successive reviews of NSP - the categories of that domain were considered complete. After dealing with redundant and missing domains, the final appraisal instrument included twenty-one domains and more specific categories (Figure 3.1).

\section{Instrument use and data analysis}

The appraisal instrument was applied to the NSP on HIV and AIDS of 21 priority countries (Table 3.1). Countries in Sub-Saharan Africa were rank-ordered based on the estimated number of people living with HIV and AIDS. The 21 countries that account for two thirds of all people living with HIV and AIDS globally were selected for inclusion in the study. Selected countries are in Sub-Saharan Africa where the HIV epidemics share similar modes of transmission. All 21 countries had at least one NSP available in English or French.

Following standard content analysis methods (USGAO, 1996; Weber, 1990), the appraisal instrument that we developed, was applied to score the NSP on HIV and 
AIDS, i.e. to score their quality and extent of evidence along the twenty-one domains of the appraisal instrument. The application of the appraisal instrument involved two phases of document review. First, the text of the documents was screened in detail to identify content related to the domains. Sentences or phrases containing information related to the domains were identified and recorded in the instrument. Second, search terms associated with the domains were entered into the search tool of the document browser to identify and highlight any evidence that may have been missed in the first screening of the documents.

After the completion of the document review, the content recorded for each domain was analyzed and classified in the relevant categories of that domain. The domain categories to which relevant content was assigned thus determined the score for that domain. For example, an NSP that included national HIV prevalence rates disaggregated by gender and age would receive a score of 2 under the domain Disaggregation of Epidemiological Indicators according to the appraisal instrument (Figure 3.1). After scoring the NSP, the individual domain scores were normalized to a range of $[0,1]$ and their sum was taken as the overall score for the NSP evaluated.

Results of the NSP scores over time were analyzed, both within countries and among all countries. Frequency of use of domains of evidence in the NSP was analyzed, as well as the variance in scores of domains of evidence. The crosscutting theme of key at-risk populations, which is relevant for multiple domains of evidence, was analyzed across countries. One particular population, men who have sex with men (MSM), was selected for focused cross-analysis. While other key populations may have larger burden or population size in particular countries, MSM were selected for the cross analysis because they are a stigmatized key population at risk of HIV present in every country. 


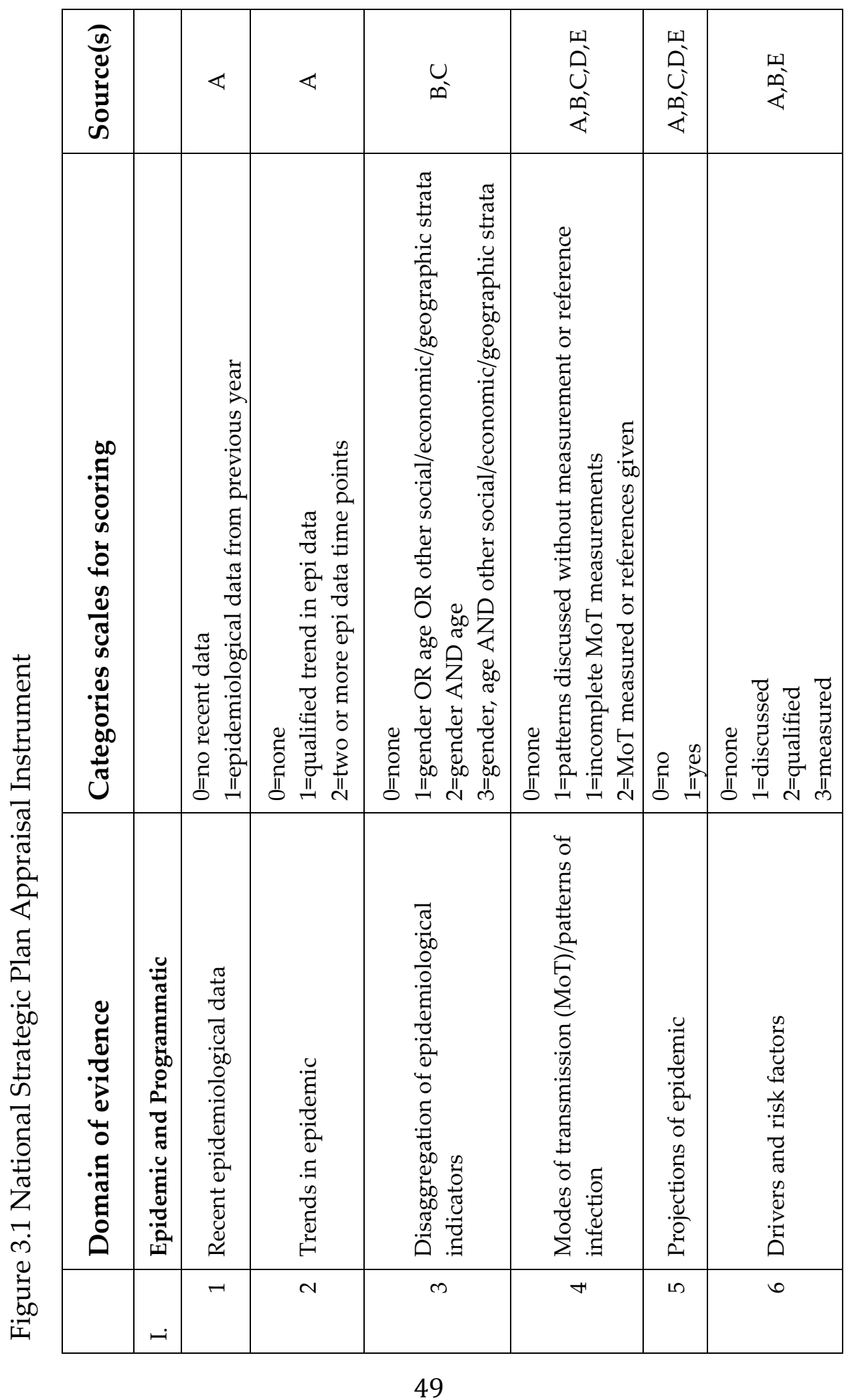




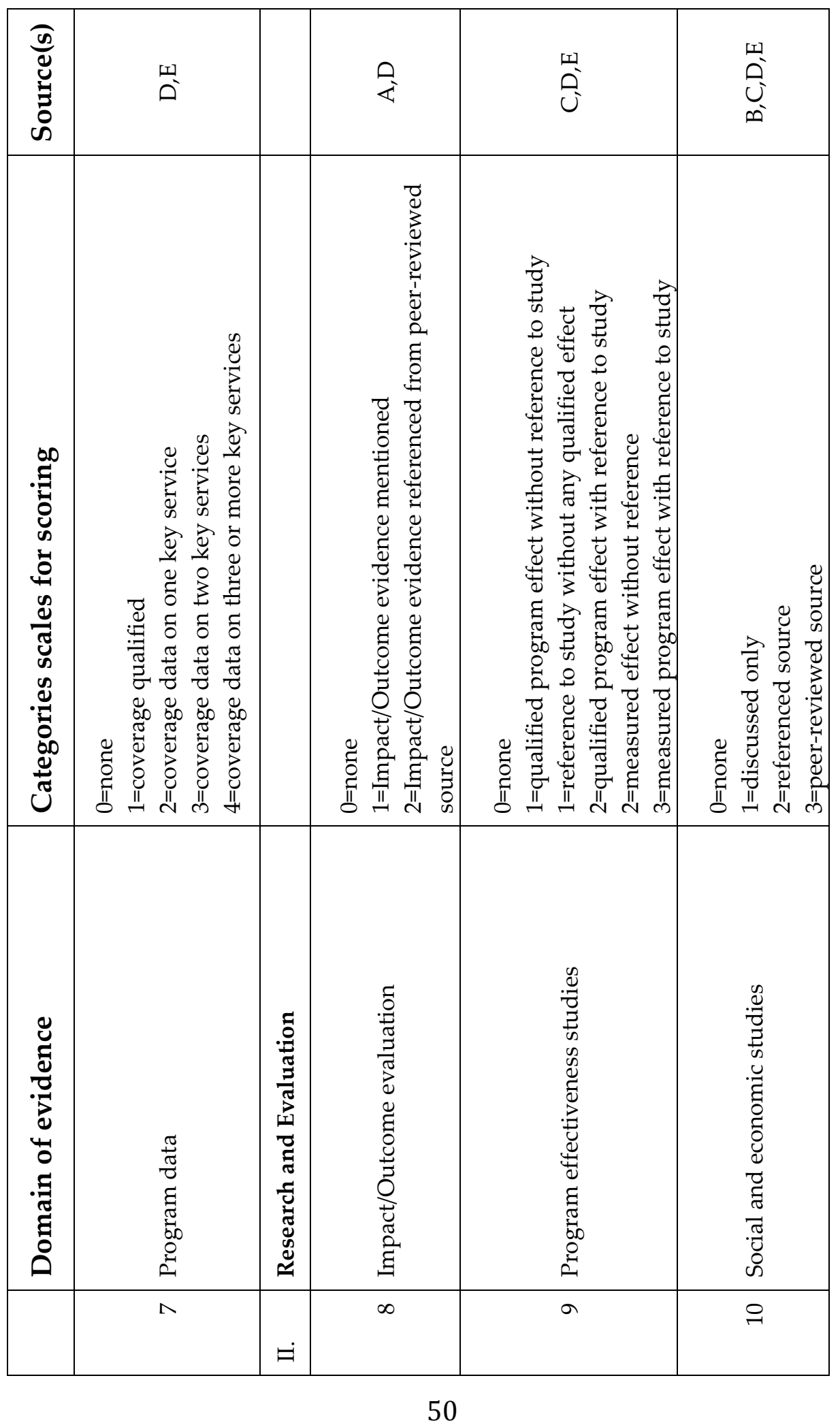




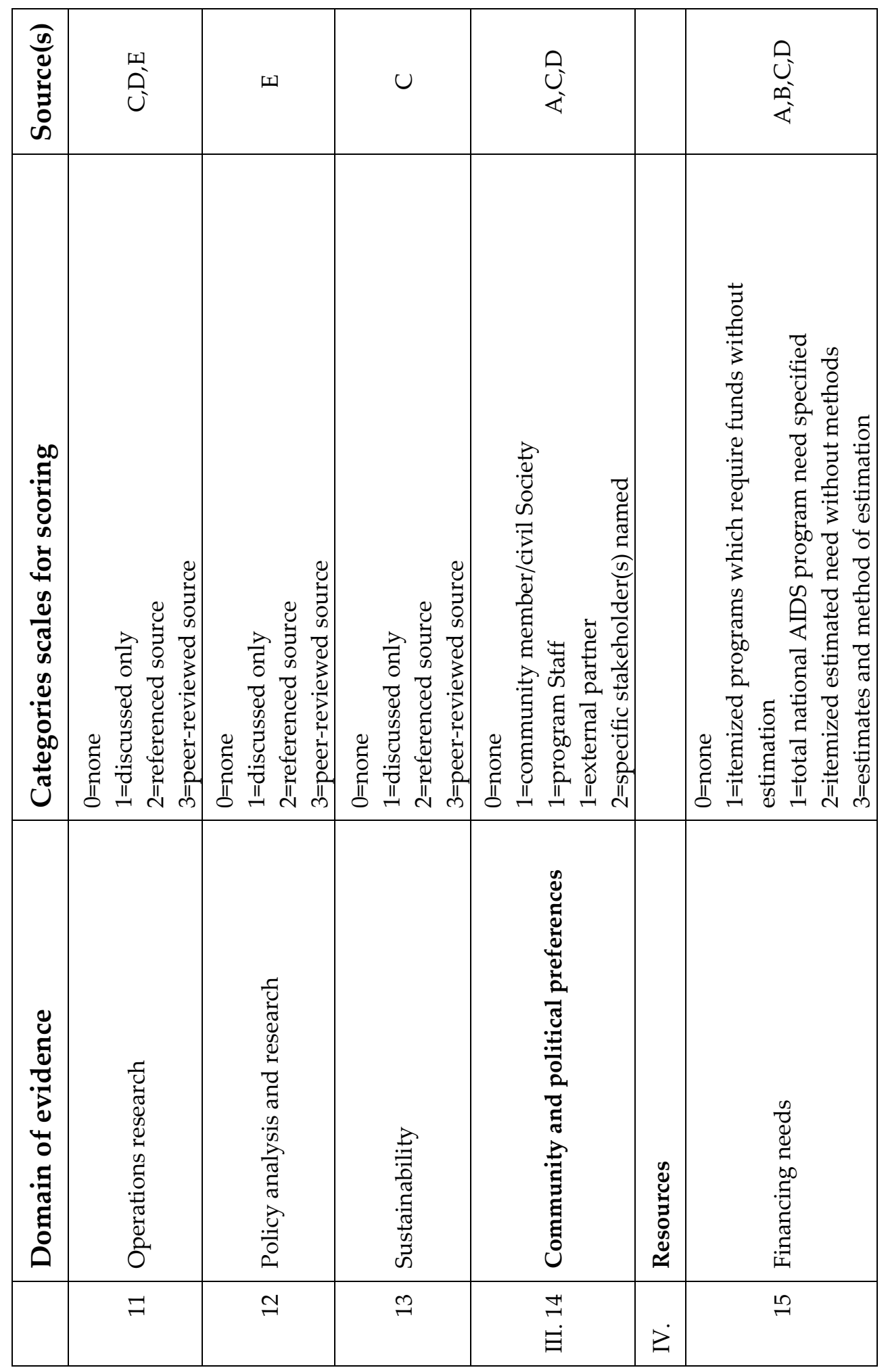




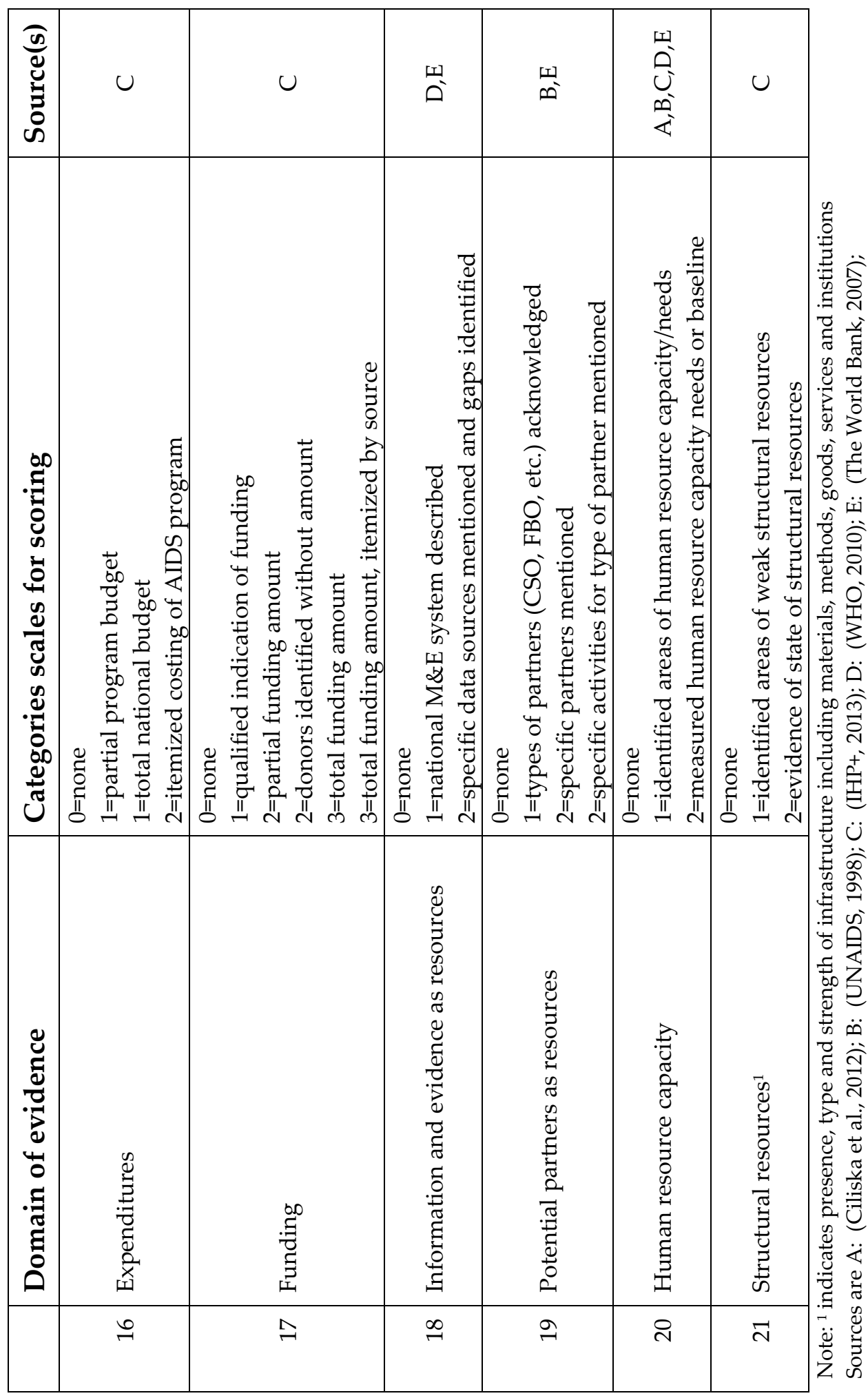


Table 3.1 Countries reviewed in this study, their estimated number of people living with HIV and AIDS (PLWHA), and their National Strategic Plans included in the review

\begin{tabular}{|c|c|c|}
\hline Country & $\begin{array}{c}\text { Estimated \# of } \\
\text { PLWHA }^{*}\end{array}$ & National Strategic Plan date(s) \\
\hline South Africa & $6,300,000$ & $2000-2005$ \\
\hline Nigeria & $3,200,000$ & 2005-2009 \\
\hline Kenya & $1,600,000$ & 2000-2005, 2005-2010 \\
\hline Mozambique & $1,600,000$ & 2010-2014 \\
\hline Uganda & $1,600,000$ & 2007-2011, 2011-2015 \\
\hline Tanzania & $1,400,000$ & 2003-2007 \\
\hline Zimbabwe & $1,400,000$ & 2006-2010, 2011-2015 \\
\hline Zambia & $1,100,000$ & 2006-2010 \\
\hline Malawi & $1,000,000$ & 2010-2012 \\
\hline Ethiopia & 790,000 & 2010-2015 \\
\hline Cameroon & 600,000 & 2011-2015 \\
\hline DR Congo & 440,000 & 2014-2017 \\
\hline Côte d'Ivoire & 370,000 & 2011-2015 \\
\hline Lesotho & 360,000 & 2011-2015 \\
\hline Botswana & 320,000 & 2003-2009 \\
\hline Angola & 250,000 & 2003-2008 \\
\hline Namibia & 250,000 & 1999-2004, 2011-2016 \\
\hline Ghana & 220,000 & 2001-2005, 2006-2010 \\
\hline Chad & 210,000 & 2012-2015 \\
\hline Rwanda & 200,000 & 2005-2009 \\
\hline Swaziland & 200,000 & 2006-2008, 2009-2014 \\
\hline
\end{tabular}

Note: * Estimated number of People Living with HIV and AIDS (PLWHA) from 2014 


\section{Reliability of the instrument}

After all NSP were scored, a random sample was rescored to check the reliability of the instrument. The reliability of the instrument was assessed using Intraclass Correlation (Rousson, Gasser, \& Seifert, 2002), which involves a single researcher rating multiple subjects (Shrout \& Fleiss, 1979). Ten NSP were selected to allow an adequate Intraclass Correlation Coefficient above 0.75 (Gwet, 2007). These ten NSP were selected at random from the set of 27 NSP and rescored following the procedure described previously.

In a reliable instrument, scores on similar items are related and consistent. This internal consistency of the appraisal instrument was tested with Cronbach's Alpha (Cronbach, 1951). Cronbach Alpha coefficients between 0.7 and 0.9 are interpreted as reliable (Gliem \& Gliem, 2003; Tavakol \& Dennick, 2011). Higher Cronbach Alpha coefficients suggest redundancies in questions, and lower coefficients suggest no correlation between the related questions that are supposed to measure a common concept. By successive removal of domains and recalculation of the Cronbach Alpha, redundant domains were identified and excluded from the instrument.

\section{Validity of the instrument}

The validity of the appraisal instrument was tested by comparing the use of evidence for planning with measures of good governance (Kaufmann, Kraay, \& Mastruzzi, 2013). Accurate information, meaningful participation of stakeholders and evidence-based planning are thought to be vital to rule of law and democratic governance (Boța-Avram, 2013; IRMT, 2002; Malyshev, 2006; Woodford \& Preston, 2013). Therefore, we expect to see a correlation between the evidence use scores and the regulatory quality index of the World Governance Indicators. Evidence 
use scores were also validated against the strength of auditing and reporting standards of the Global Competitiveness Index from the World Economic Forum (WEF, 2014). Both of these measures rely on functioning monitoring systems and availability of evidence.

In correlation analysis of the evidence use scores and regulatory quality dimension of the World Governance Indicators, NSP of Democratic Republic of Congo was excluded since no World Governance Indicator values are available for a corresponding year. Outliers were identified and removed using the Tukey method (Tukey, 1977). All external validity tests were coded and tested in the statistical package R, version 3.0.2 (R Core Team, 2013).

\subsection{Results}

The 27 NSP on HIV and AIDS were rated using the appraisal instrument developed in this study. The mean score received by the rated NSP was 9.1 out of a maximum of 21 points, where the maximum score represents an NSP with perfect information along all 21 distinct domains of evidence measured by the instrument. The 2011-2015 NSP from Zimbabwe received the highest score of 14.83, and the 2003-2007 NSP from Tanzania received the lowest score of 4.25.

In checking the reliability of the instrument, rescoring of ten NSP resulted in a mean score of 8.5 compared to a mean score of 9.0 in initial scoring of the same set. Scatterplot of the initial scores against the rescores showed a definite trend (Figure 3.2), although offset from the zero intercept. These differences were likely to be systematic errors due to the rater becoming familiar with the NSP being rated, also called the learning effect of the rater (Rousson et al., 2002). Calculation of the 
Intraclass Correlation Coefficient resulted in a coefficient value of $0.88,95 \% \mathrm{CI}$ [0.59 - 0.97], which is interpreted as sufficient reliability of the appraisal instrument. Analysis of internal consistency of the instrument demonstrated acceptable reliability, with the Cronbach's Alpha coefficient calculated at 0.75.

Figure 3.2 Scatterplot of intra-rater agreement in use of instrument to assess evidence use in NSP

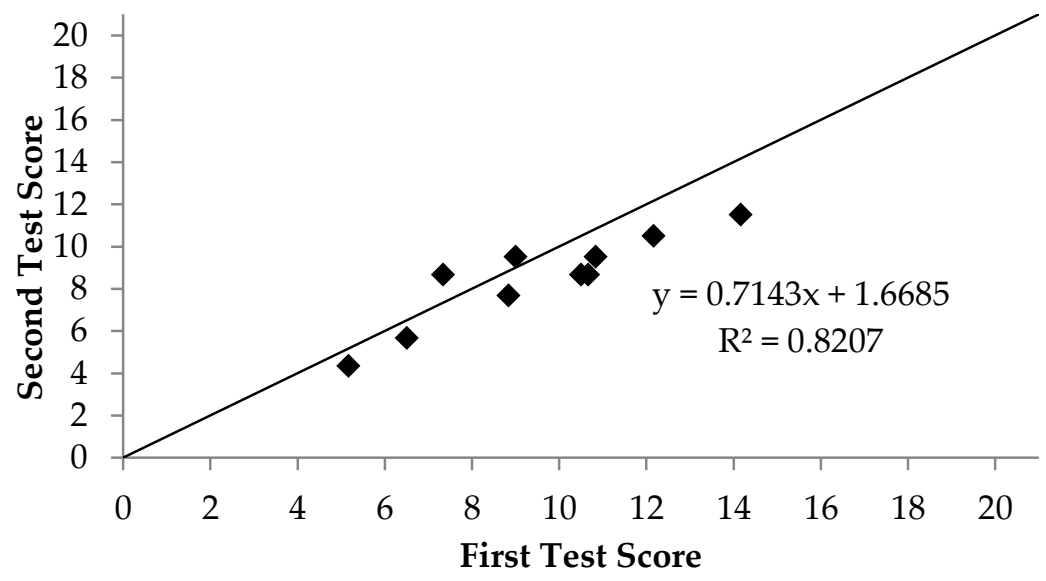

Note: Identity line is plotted as a reference.

In testing the validity of the instrument, the regulatory quality index of World Governance Indicator showed a strong association with the evidence use scores (Spearman's rho coefficients of 0.54, $\mathrm{p}<0.05)$. This World Governance Indicator can be interpreted as being strongly correlated with the evidence use score produced by the appraisal instrument. Auditing and Reporting Index of the Global Competitiveness Index indicators had a strong correlation with evidence use (Spearman's rho coefficient of $0.48, \mathrm{r}=0.086$ ).

There is a noticeable trend in increasing scores over the years (Figure 3.3), indicating that the types of evidence used in NSP are more diverse and the NSP 
quality is greater today than it was in the past. The increasing trend in the score over time is observed over all NSP reviewed, but also within successive NSP of four countries. Only one country, Uganda, showed a decline in the score over successive NSP.

Figure 3.3 Appraisal instrument scores on quality and use of evidence in NSP over time

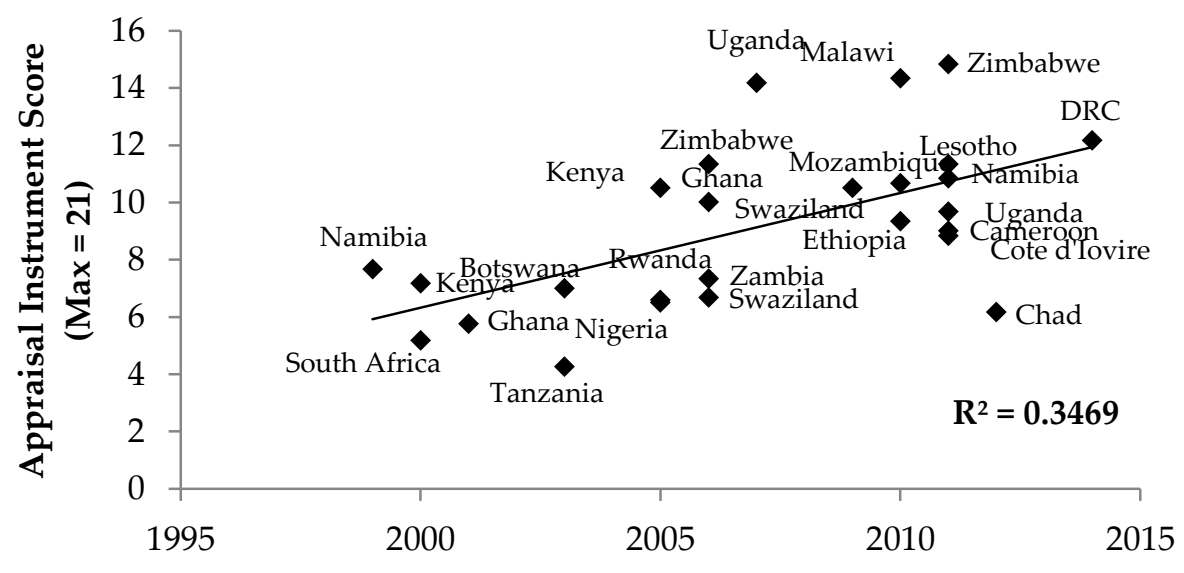

Beginning Year of National Strategic Plan Period

Data on Epidemic Drivers and Risk Factors was the highest scored domain of evidence used in the NSP. It appeared in some form in every NSP reviewed. Expenditure Data and Impact/Outcome Evaluation were equally the lowest scored domains of evidence used in the NSP (Table 3.2). When domains of evidence were clustered around four themes (Figure 3.1), the Epidemic and Programmatic cluster had the highest score followed by Community and Political Preferences and Resources clusters. The Research and Evaluation evidence domain cluster had the lowest score and also the lowest variance on its cluster score. Modes of Transmission/Patterns of Infection and Information and Evidence as Resources were two domains of evidence that made the greatest difference in rating between 
the bottom quartile and top quartile of scored NSP. Seven of the 27 NSP did not include participation of Civil Society in the planning process. Six of the NSP did not include participation of Non-Governmental Organizations.

Table 3.2 Domains of evidence and mean score received for NSP rated using the appraisal instrument

\begin{tabular}{lcc}
\hline Domain Item & Mean Score & $\begin{array}{c}\text { Score } \\
\text { Variance }\end{array}$ \\
\hline Drivers and risk factors & 0.81 & 0.07 \\
Program data & 0.75 & 0.14 \\
Recent epidemiological data & 0.65 & 0.12 \\
Potential partners as resources & 0.65 & 0.14 \\
Community and political preferences & 0.58 & 0.12 \\
Trends in epidemic & 0.56 & 0.19 \\
Modes of transmission/patterns of infection & 0.56 & 0.19 \\
Disaggregation of epidemiological indicators & 0.56 & 0.09 \\
Financing needs & 0.54 & 0.16 \\
Human resource capacity & 0.54 & 0.15 \\
Funding & 0.47 & 0.17 \\
Information and evidence as resources & 0.41 & 0.15 \\
Social and economic studies & 0.36 & 0.06 \\
Structural resources & 0.33 & 0.09 \\
Projections of epidemic & 0.26 & 0.19 \\
Program effectiveness studies & 0.21 & 0.08 \\
Policy analysis and research & 0.21 & 0.03 \\
Sustainability & 0.21 & 0.03 \\
Operations research & 0.20 & 0.04 \\
Impact/Outcome evaluation & 0.13 & 0.07 \\
Expenditures & 0.13 & 0.09 \\
\hline
\end{tabular}

Four domains of evidence had search terms related to key populations at risk of HIV or key populations were a category in these domains of evidence. These four domains of evidence - Disaggregation of Epidemiological Data, Modes of 
Transmission, Community and Political Preferences, and Potential Partners as Resources - had mean scores above the median. These four domains of evidence also had the highest score variance across the countries. One key population at risk, MSM, was selected for further analysis in the domains of evidence in the NSP. Only three out of the 27 NSP reviewed demonstrated any evidence for MSM, and only one NSP reported the size of this key population. Ten NSP did not mention MSM at all, and four mentioned that despite being a key population, evidence is lacking for MSM. Two NSP stated that homosexuality is illegal and therefore no data is available for MSM.

\subsection{Discussion}

This study builds on previous research that suggests investments in monitoring and evaluation systems are paying off in terms of improved generation of evidence (Alfven et al., 2014; Peersman et al., 2009). It overcomes some of the limitations of those studies in the use of self-reported data over a short period of time, with an objective analysis of use of evidence for planning based on validated instruments. Our results further suggest that with regard to NSP, evidence is not only being generated, but also used in planning, and that evidence use in NSP is increasing over the years.

There is still a gap in reaching optimal levels of evidence use in NSP, with scores of recent plans remaining below the midpoint of the appraisal instrument maximum score. The results indicate that there is more evidence used in NSP in terms of surveillance of the epidemic and monitoring of HIV programs than in research and evaluation. Evidence from evaluation and research are noticeably absent in the plans, particularly impact evaluations and operations research. These results are 
consistent with expert opinion that evaluation is a neglected area of health programs, particularly in HIV and AIDS (Bennett, Boerma, \& Brugha, 2006; Boerma \& Stansfield, 2007). However, a recent survey and evaluation gap analysis by the International Initiative for Impact Evaluation (3ie) indicated that there is a strong evidence base on the effectiveness of HIV programs, with Sub-Saharan Africa being a leader in impact evaluations (Rankin, Heard, \& Diaz, 2016). An explanation for these contradictions may be that although evaluative evidence is available, it is not generated in a timeframe aligned with the national strategic planning process to be used effectively (Oliver, Innvar, Lorenc, Woodman, \& Thomas, 2014).

Overall, there is adequate evidence related to key populations in the NSP. However, there is also great variance across NSP in scores on evidence that related to key populations at risk of HIV. A particular gap observed is in data on MSM, which may be due to lack of evidence generation activities for this key population at risk of HIV, or lack of will to use the available data. The 3ie has developed an Evidence Gap Map, which highlights where few or no recent, high-quality impact evaluations or systematic reviews exist on particular topics. Their report similarly observes that LGBTQ adolescents are a top group with weak programming evidence (Rankin et al., 2016).

Given the remaining gap in evidence, the appraisal instrument can serve the NSP development process. National steering committees, setup to develop the plans, may request reviews of previous NSP using the appraisal instrument to learn about areas of weakness in evidence use and quality. The reviewers can then make recommendations to either improve evidence generation where there are gaps in quality evidence, or improved use of evidence where evidence exists but is not used. 
Before signing a grant agreement, the Global Fund uses a Capacity Assessment Tool (CAT) to collect information and rate the capacity and systems in place to implement the grant, including monitoring and evaluation systems (The Global Fund, 2015). While the CAT supports self-reporting on the strength of monitoring and evaluation systems, we believe the instrument developed in this study adds objective measures of the strength of NSP. Considering the strength of evidence used in NSP as an indicator of monitoring and evaluation performance, the appraisal instrument presented here can supplement the CAT, and support grant allocation decisions by the Global Fund.

Funding decisions of many development agencies and donors, like the Global Fund, Global Alliance for Vaccines and Immunization, European Commission, and Millennium Challenge Account, are tied to evidence-based planning and interventions (The Global Fund, 2009). Meanwhile, donor funding for the HIV response is expected to decrease (The Kaiser Family Foundation \& UNAIDS, 2013). As a result, the economic incentive to use evidence for planning will decrease in countries that rely on foreign aid for their HIV response. As countries move toward domestic financing of monitoring and evaluation, the influence of donors and development partners on the decision-making process are also expected to reduce. Integration of HIV into the broader health sector also implies that some elements of HIV monitoring systems may become weak in the process, while sustainability of the overall Health and HIV response gain strength from integration.

To maintain the gains in evidence-based planning, governments will need to remain vigilant in the generation and use of information for planning at policy, program, and facility levels. At the global level, the incentive for a continued use of 
evidence could be created by including measures of evidence-based planning in international benchmarks of governance, which are set as targets in national development plans. Countries aiming to increase their governance score, in order to attract foreign investments, may set a strategy to improve the use of evidencebased planning. In addition, by standardizing the indicators in the appraisal instrument across countries, development agencies and donors can better allocate resources to countries and domains based on evidence generation capacity and use. When considering incentives for evidence-based planning, the use of evidence as well as the effectiveness of those plans should be considered, in order to prevent situations of rewarding words without action. Evidence use, and its possible association with optimal decisions, in lower- and middle-income countries should also be compared with high-income countries (Nutbeam, 2004; Sanderson, 2009; K. Smith, 2013).

The instrument developed in this study was assessed for its reliability and external validity. The significant correlations with indicators of regulatory quality, auditing and reporting, which rely on strong availability and use of evidence, validate the results seen in scoring of evidence use in NSP. Although scores in the retest of the NSP differed slightly from the initial test, the direction and scale of the difference suggests that it is likely due to the rater becoming familiar with the NSP being rated. Therefore, the instrument can be said to be sufficiently reliable.

The instrument developed in this chapter applies content analysis methods to review NSP. Since there are a number of existing tools that our instrument is built upon, we are not working from a completely naïve perspective that is the hallmark of conventional content analysis (Hsieh \& Shannon, 2005). However, the approach of mapping existing tools to develop the instrument does come with the challenge of a bias toward the existing domains of evidence, and blinding the researcher 
from other possible categories. Content analysis of the NSP provides basic insights on use of quality evidence, but there is no broader analysis of the context of the NSP. Therefore the results of the instrument rely on the credibility and reliability of the instrument and the users of the instrument.

Due to resource limitations, only one individual, who was involved in the development of the instrument, rated the NSP in this study. While we strived to make the instrument objective in its assessment of evidence quality and use in NSP, we do not know if other raters would score the same NSP differently. The instrument ultimately depends on those who apply it to use it correctly to say to what extent quality evidence was used in the NSP.

Our study considers the elements of evidence use in NSP, and involvement of practitioners and community members in NSP development, although it does not further investigate the representativeness of the evidence, use of the best available evidence, or critical assessment of the evidence, that would qualify it as “intelligent" policy making (Deas, Mattu, \& Gnich, 2013; Nutbeam, 2004; Sanderson, 2009).

\subsection{Conclusion}

Decision-making in the absence of evidence is not optimal. However, decisionmaking with an abundance of evidence, criteria, and stakeholders is no simple task either. With numerous criteria for decision-making, policymakers have a challenging task of sorting through available information and making optimal decisions to achieve the desired health and social outcomes. However, human 
beings are limited in their ability to make rational decisions when confronted by an overload of scenarios.

This chapter has identified a lack of evidence from evaluation and research in the NSP, which may benefit from better alignment in timing of evaluations with the national strategic planning process. While this chapter shows that available evidence is being used in planning, it cannot prove that optimal decisions are being made with the evidence. Further longitudinal studies are needed to plausibly associate evidence use in NSP with optimal decisions. Moreover, evaluation is needed to see if implementation based on the NSP reflects the use of evidence in the NSP, and how well or poorly those plans perform when based on evidence, compared to ad-hoc planned interventions (Klein, 2000; Wye et al., 2015). To overcome the individual errors and also to ensure inter-rater agreement among raters, several raters should apply the instrument, and the consistency of their scores should be tested.

The appraisal instrument in this chapter was only applied to national plans. However, the instrument may also be applied to agency specific plans to assess the level of use of evidence. Comparison of the use of evidence in planning by agencies can reveal the variations and consistency in breadth of evidence use, and characterize the evidence-based planning culture of agencies. The Country Operation Plan of the U.S. President's Emergency Plan for AIDS Relief (PEPFAR) is one potential candidate for assessment of evidence use vis-à-vis evidence use in NSP (PEPFAR, 2015).

Evaluation of the national planning process before and after an evidence generating activity could help identify barriers and enablers to evidence use at the national level. The appraisal instrument developed in this study may provide the 
metrics to measure change in evidence use in NSP. Of interest is the effect of community ownership and involvement in evidence generation on the scale of its use in planning and policy making, particularly in countries identified as lacking evidence for key populations at risk of HIV. Actions along these lines could contribute to realizing the potential of evidence to improve quality of policy decisions and plans. 


\section{Chapter 4: Choices in Surveillance of HIV: Lessons from Viet Nam}

This chapter draws upon:

Safarnejad A, Groot W, Pavlova M. Choices in Surveillance of HIV: Lessons from Viet Nam. Submitted for publication. 


\section{Abstract}

Estimation of the size of populations at high risk of HIV is a key activity in the surveillance of the HIV epidemic. The existing framework for considering future research needs may provide decision-makers with a basis for a fair process of deciding on the methods of the estimation of the size of key populations at risk of HIV. This study explores the extent to which stakeholders involved with population size estimation agree with this framework, and thus study updates the framework.

We conducted 16 in-depth interviews with key informants from city and provincial governments, NGOs, research institutes, and the community of people at risk of HIV. Transcripts were analyzed and reviewed for significant statements pertaining to criteria. Variations and agreement around criteria were analyzed, and emerging criteria were validated against the existing framework.

Eleven themes emerged which are relevant to the estimation of the size of populations at risk of $\mathrm{HIV}$ in Viet Nam. Findings on missing criteria, inclusive participation, community perspectives and conflicting weight and direction of criteria provide insights for an improved framework for the prioritization of population size estimation methods.

The findings suggest that the exclusion of community members from decisionmaking on population size estimation methods in Viet Nam may affect the validity, use, and efficiency of the evidence generated. However, a wider group of decision-makers may introduce diverse definitions, weight and direction of criteria. Although findings here may not apply to every country, the principles of fair decision-making, value of community participation in decision-making and the expected challenges faced, merit consideration in every situation. 


\subsection{Introduction}

Estimation of the size of populations at risk of HIV is a key activity in the surveillance of the HIV epidemic and management of the response. Key populations at risk of HIV include, but are not limited to, men who have sex with men, people who inject drugs, and sex workers. Estimation of the size of these key populations is used in three areas of a national HIV response: policy, intervention, and research. In the first area, policymakers use size estimation data to advocate for, mobilize resources for, and prioritize prevention and care programs targeted at key populations at risk of HIV. The second area concerns organizations involved in interventions for key populations at risk of HIV, such as providing clean needle and syringe distribution to people who inject drugs. These organizations need to know the size of their target population in order to plan for, and provide adequate services to particular sub-populations, and monitor the performance of their activities. In the third area, researchers make use of size estimates in evaluating the impact of interventions for key populations at risk of HIV on the overall HIV epidemic, and recommending ways to shift from pilot projects to achieving larger scale coverage of HIV prevention and care programs (UNAIDS, IMPACT, \& FHI, 2003; UNAIDS \& WHO, 2010; Vandepitte et al., 2006).

Viet Nam is a country with a concentrated HIV epidemic, with an estimated $0.4 \%$ prevalence of HIV among the adult population, an incidence of 0.21 per 1,000 population, 8,600 AIDS-related deaths, and approximately 110,000 people living HIV receiving anti-retroviral treatment in 2015 (UNAIDS, 2015). HIV prevention, treatment, care and support services are managed nationally by the Viet Nam Administration of HIV/AIDS Prevention and Control in the Ministry of Health with support from multilateral agencies such as the Global Fund, and bilateral programs such as the United States President's Emergency Plan for AIDS Relief 
(PEPFAR) (Vietnam Ministry of Health, 2006). The HIV surveillance system in Viet Nam was established in the early 1990s collecting epidemiological and behavioral data (Viet Nam Ministry of Health, 2007; Vietnam Ministry of Health, 2006). Among the surveillance activities, a number of estimates of the size of key population at risk of HIV were attempted in Viet Nam (Hien, Long, \& Huan, 2004; Nadol, 2012; Tuan, Tuan, Thanh, Quang, \& Sabin, 2014). Some of these methods estimate the key at-risk population sizes based on a simple multiplier of the general population, using assumptions developed by the Viet Nam HIV estimates and projection technical working group (Nadol, 2012). Other methods use police census information or program data from the Ministry of Labor, Invalid and Social Affairs working with drug users to estimate the size of populations at risk of HIV (Hien et al., 2004; Nadol, 2012). More recently, capture-recapture and multiplier methods have been applied to estimate the size of populations at risk of HIV (Safarnejad, Nga, \& Son, 2017; Tuan et al., 2014). Still other methods of key at-risk population size estimation with a number of design decisions exist that have not yet been tried in Viet Nam, such as the network scale-up method, the surveysurveillance discrepancy method, or the "never married" method (Catania, Canchola, \& Pollack, 2002; Marcus, Hickson, Weatherburn, \& Schmidt, 2013; UNAIDS \& WHO, 2010).

The concurrent use of multiple methods of size estimation has been justified to validate and interpret the results (Abdul-Quader, Baughman, \& Hladik, 2014; WHO, 2014a; Yu, Calleja, Zhao, Reddy, \& Seguy, 2014). However, in transitioning economies like Viet Nam, where funding for HIV programs by donors is rapidly decreasing, and the increase in national funding is unable to keep pace to cover the funding gaps, difficult choices are faced in prioritizing HIV surveillance activities such as population size estimation. The limits to the magnitude of resources that can be spent on surveillance constrains the national HIV program's ability to 
conduct population size estimation studies with multiple concurrent methods (Magnani, Sabin, Saidel, \& Heckathorn, 2005).

Financial cost constraints are not the only force driving decisions in choice of population size estimation method. Decision-makers must also consider the social costs of their decisions related to the methods of surveillance (Center for Disease Control and Prevention, 2010; Lussier, Richard, Bennett, Williamson, \& Nagpurkar, 2012), and specifically methods of population size estimation (Herek, Capitanio, \& Widaman, 2003; Herek, Kimmel, Amaro, \& Melton, 1991; Matthews \& Velleman, 1997). Social costs can include for example perpetuation of stigma and discrimination toward marginalized groups, such as men who have sex with men, and people living with HIV $(\mathrm{GNP}+$, 2016). For example, in examining the link between AIDS stigma and support for name-based reporting, Herek et al. highlight that such policies in surveillance "may evoke anxiety and encounter resistance to the extent that it is perceived as insensitive to - or even fostering - preexisting AIDS stigma" (Herek et al., 2003).

As decision-makers are being confined to deciding on the "right" size estimation method (Vadivoo et al., 2008), fair and explicit consideration of a broad set of criteria for prioritization of population size estimation methods becomes imperative. A number of comprehensive approaches exist for health program and research priority setting that define procedures for eliciting criteria and dealing with conflicting criteria (Rudan et al., 2010). Accountability for Reasonableness (A4R), Combined Approach Matrix (CAM), and Interactive Technology Assessment (iTA) are examples of such approaches (Daniels \& Sabin, 2000; Ghaffar, Collins, Matlin, \& Olifson, 2009; Grin, Graaf, \& Hoppe, 1997). Essential National Health Research (ENHR) and the Council on Health Research for Development (COHRED) also provide guidance which has been used in 
prioritizing health research in developing countries (Montorzi, Haan, \& IJsselmuiden, 2010; Okello, Chongtrakul, \& The COHRED Working Group on Priority Setting, 2000; Rudan et al., 2010).

Despite availability of these approaches many health priority setting exercises develop their own, unique methods, because of contextual particularities of priority setting (Viergever et al., 2010). The Framework for Considering Study Designs for Future Research Needs developed by the Agency for Healthcare Research and Quality (AHRQ) is one framework that specifically considers different study designs for future research needs (Carey et al., 2012). The AHRQ framework is intended to standardize the terminology and process in prioritizing health research. The two salient features of the AHRQ framework that distinguish it from the aforementioned health priority setting approaches and make it appropriate for use in this study, are the focus on prioritizing both research and methods of research, and the explicit articulation of criteria related to the selection of research design and methods. In a series of methods papers, AHRQ recommends some criteria and procedures for consistent application in the selection of research design for future research needs (Andrews, 2013; O'Haire et al., 2011). The framework is not intended to be prescriptive, and it lacks a clear description of stakeholder involvement in deliberations, or processes to deal with conflicts and dependencies of criteria. Although the framework has been successfully used in the United States, to our knowledge it has not been applied in developing countries. This framework can potentially be relevant for evaluating the appropriateness of the design of a study focused on size estimation in Viet Nam.

The use of the AHRQ framework is more so appropriate for Viet Nam as it can help to improve accountability and participation in Viet Nam's HIV Strategy and 
HIV surveillance activities including population size estimation (National Committee for AIDS Drug and Prostitution Prevention and Control, 2012; Viet Nam Administration of HIV/AIDS Control, 2007). Participation of multiple stakeholders (including members of the community who have a stake in the decisions made) to elicit explicit and transparent criteria that play a part in making decisions, is a precondition for a fair priority setting process (Daniels \& Sabin, 1997, 2000). Involvement of multiple stakeholders in decision-making processes is grounded in democratic theory (Abelson, Forest, et al., 2003; Scheer, Garcia, Laan, Burg, \& Boenink, 2014; UNAIDS, 2010a) and the constructivist tradition (Leys, 2003). The process of research priority setting frequently engages researchers and government but meaningful involvement of other key stakeholders are less frequent (McGregor, Henderson, \& Kaldor, 2014; Tromp, Prawiranegara, Subhan Riparev, et al., 2015). A recent review of 27 national HIV plans found that only 9 plans had specified the community group or civil society involved in the planning process (Chapter 3). One reason for limited participation of these stakeholders in decision-making processes is the risk of not reaching consensus, which may lead to less acceptance and trust in the results. Another reason for the limited stakeholders' participation is that stakeholders, particularly non-expert members of the community, often perceive their values and arguments are not properly considered in national HIV plans.

The AHRQ framework may provide a basis for a fair process of deciding on the HIV surveillance methods including methods of estimation of the size of key populations at risk of HIV. This study aims to explore the extent to which diverse stakeholders involved with HIV surveillance agree with the AHRQ framework. In doing so, we will update the framework, and contextualize it for the problem of deciding on a method of size estimation for key populations at risk of HIV in Viet Nam. An updated framework based on a wide stakeholder involvement will 
facilitate its use in a fair decision-making process, conforming to the A4R framework (Daniels \& Sabin, 1997, 2000). Findings of this study may give other transitioning-economy countries insights into relevant criteria for prioritization of population size estimation methods among other HIV surveillance activities and the role of different stakeholder in that decision-making process.

\subsection{Methods}

\section{Study design}

We designed a qualitative study to capture various perspectives of the size estimation process, including selection of appropriate method, implementation of the method, and use of the generated evidence. The study took place in 2015 in Ho Chi Minh City and Vinh City. Ho Chi Minh City is a highly urbanized city, with the largest population in Viet Nam (7,123,340 inhabitants in 2009) (Vietnam Ministry of Planning and Investment \& UNFPA, 2010), whereas Vinh City is a provincial city of 230,000 inhabitants and it is considered to be one of the poorest cities in Viet Nam (Sustainable Cities Programme, United Nations Human Settlements Programme, \& United Nations Environment Programme, 2002). The data collection method of the study was in-depth interviews with key informants who were previously involved in population size estimation studies.

\section{Key informants}

The study used a purposive, non-random sampling strategy. Since there is no hypothesis being tested and no associated level of confidence in any test results in this study, the number of key informants was not specifically pre-defined. The focus was on reaching as many informants as possible within a pre-defined period. 
The key informants were selected through personal contacts based on criteria of availability, subject matter knowledge, and representation of the diversity of stakeholders. In each city, we intended to identify one health program manager representing the Provincial AIDS Program, one technical expert from an NGO or research institute involved in surveillance, and one individual from the community of people at risk of HIV in Viet Nam. The motivation behind selecting these three groups was that they encompass the actors who have a stake in the decisionmaking in surveillance activities, including population size estimations, at the provincial level in Viet Nam. These three groups - representing government, research and development partners, and community members - are referenced by the Greater Involvement of People Living with HIV principle formalized at the 1994 Paris AIDS Summit, and also reflect the membership structure of the Country Coordinating Mechanism of the Global Fund at the central level (GFATM, 2013; Joint United Nations Programme on HIV/AIDS, 1999).

As this qualitative study ran concurrently with a size estimation demonstration pilot, participants in that study facilitated the selection of key informants for this study. Following the first interviews, the key informants were asked to nominate other candidates from their organization or network to be interviewed. We asked the key informants to suggest individuals who would be representative of their peers and who would be likely to speak candidly with us. No individuals refused to participate. We completed 16 in-depth interviews in total.

\section{Data collection}

Verbal and written information about the study were given to each potential key informant. Participation was voluntary and the respondents were informed that they could withdraw at any time and that all data would be treated confidentially. 
Individual verbal informed consent was obtained from the participant at the beginning of each interview. The survey protocol and instrument material used in this study, and the concurrent population size estimation pilot, were reviewed and approved by the Institutional Review Board of the Hanoi School of Public Health (Hanoi, Viet Nam). An interview guide with open-ended questions aided the focus of the interviews (see Appendix $G$ for an outline of the topics and probing questions). There is flexibility in the interview guide to offer space for key informants to raise other issues that they might consider to be pertinent. A table describing the methods of size estimation and the acknowledged strengths and weakness along common criteria established by standards setting bodies, was introduced to the key informants to aid the interviews (Appendix H). All interviews were conducted in Vietnamese and later transcribed in English.

The interviews followed an informal format. Pre-defined questions in the interview guide directed the conversation to those topics that matter to the study, while ad hoc questions followed the direction of the conversation. The interviews were conducted while interviewer and key informant were seated at a public or private location chosen by the key informant. The procedures and setting, and the existing relationships of the research team with the key informants made the interviews similar to a 'conversation with a purpose' (Burgess, 1984; Carpiano, 2009). The approach created an open situation in which experiences and perceptions (positive and negative) could be openly shared, without the key informants fearing they were being too critical. However, all respondents were assured of the confidentiality of the data and that the interviews are intended to be a nonjudgmental but formative learning opportunities.

Trained investigators with experience in in-depth interviewing for qualitative research conducted the interviews. Interviews lasted from 30 to 90 minutes. All 
interviews were conducted in person and audio recorded (with the key informants' consent) and were transcribed verbatim. The transcripts were translated to English by the interviewers. Interviews were conducted in August-November, 2015. The authors' experience and observation as a participant in discussions and decisions on methods of surveillance were also used as an input to the exploration of the research questions in this study.

\section{Analysis}

Transcripts were analyzed according to qualitative research guidelines. Transcripts were read several times by one investigator to search for and code the key informant's most significant statements pertaining to criteria. The emerging codes were recorded in a codebook, which included a compilation of the codes, illustrative quotes attributed to respondent profiles, and statements that guided the use of the code. Codes that seemed to have similarities were grouped into thematic patterns based on the consensus of all three investigators. Disagreements about grouping of codes into thematic patterns were resolved through a discussion until consensus was reached. There were no occasions that consensus could not be reached. The emerging themes were documented and maintained as a permanent record of our analysis progress. These steps were repeated until no new themes emerged. Criteria for choosing the population size estimation methods were extracted from the emerging themes, arrayed by the profiles of the key informants. This combined process allowed us to compare and contrast themes within and between the different key informant profiles and different sites. Comparison and contrast of views of themes between key informants also involved identifying sources of variation or agreement. Emerging criteria were validated against the criteria in the AHRQ framework. 


\subsection{Results}

In-depth interviews were carried out with 16 key informants. There were five key informants representing the government, three key informants representing NGOs and research institutes, and eight key informants representing the community of people at risk of HIV. There were an equal number of key informants participating from Vinh City and Ho Chi Minh City. Information on location of key informant interviews and their group membership is provided in Table 4.1.

Table 4.1 Key informants location of interview and group membership

\begin{tabular}{lll}
\hline Key informant & Location of interview & Group membership \\
\hline 1 & Vinh City & Government \\
2 & Vinh City & Government \\
3 & Vinh City & Government \\
4 & Vinh City & NGO/Research Institute \\
5 & Vinh City & NGO/Research Institute \\
6 & Vinh City & Community \\
7 & Vinh City & Community \\
8 & Vinh City & Community \\
9 & Ho Chi Minh City & Government \\
10 & Ho Chi Minh City & Government \\
11 & Ho Chi Minh City & NGO/Research Institute \\
12 & Ho Chi Minh City & Community \\
13 & Ho Chi Minh City & Community \\
14 & Ho Chi Minh City & Community \\
15 & Ho Chi Minh City & Community \\
16 & Ho Chi Minh City & Community \\
\hline
\end{tabular}

In the analysis of the data collected in the interviews, 11 themes emerged as having particular relevance to the process of selecting the method for the estimation of the 
size of populations at risk of HIV. Table 4.2 summarizes the 11 emerging criteria theme and how they relate to the criteria in the AHRQ framework. In the following sections, for each criteria theme, we present the summary of findings, along with quotations from key informants that express common views or concepts. The criteria themes are ordered alphabetically for easier reference.

\section{Appropriate for the community}

Key informants shared several concerns that were grouped under the appropriate for the community criterion. Less than half of all key informants interviewed mentioned this criterion $(n=7)$. But within the sub-group of eight key informants, who are also at-risk population community members, the majority mentioned this criterion $(n=6)$. The most common concerns were about methods that make the subjects in size estimation studies feel uncomfortable and stigmatized. For example, strangers calling gay individuals at their home and asking detailed questions about their sexual behavior. Another concern was about the type and value of incentives given to participants in population size studies. One key informant talking about incentives given to people who inject drug to participate in surveys said, "they don't want mobile phone credit, they want money". When asked why they want money, the key informant said, "to buy their next dose of drug." The question of what is appropriate for the community to ensure participation in the population size estimation remains a valid criterion to consider alongside others. This criterion is closely linked with the community participation criterion (see below), in that the involvement of peers in the studies helps to avoid the situation of participants being called by strangers. 


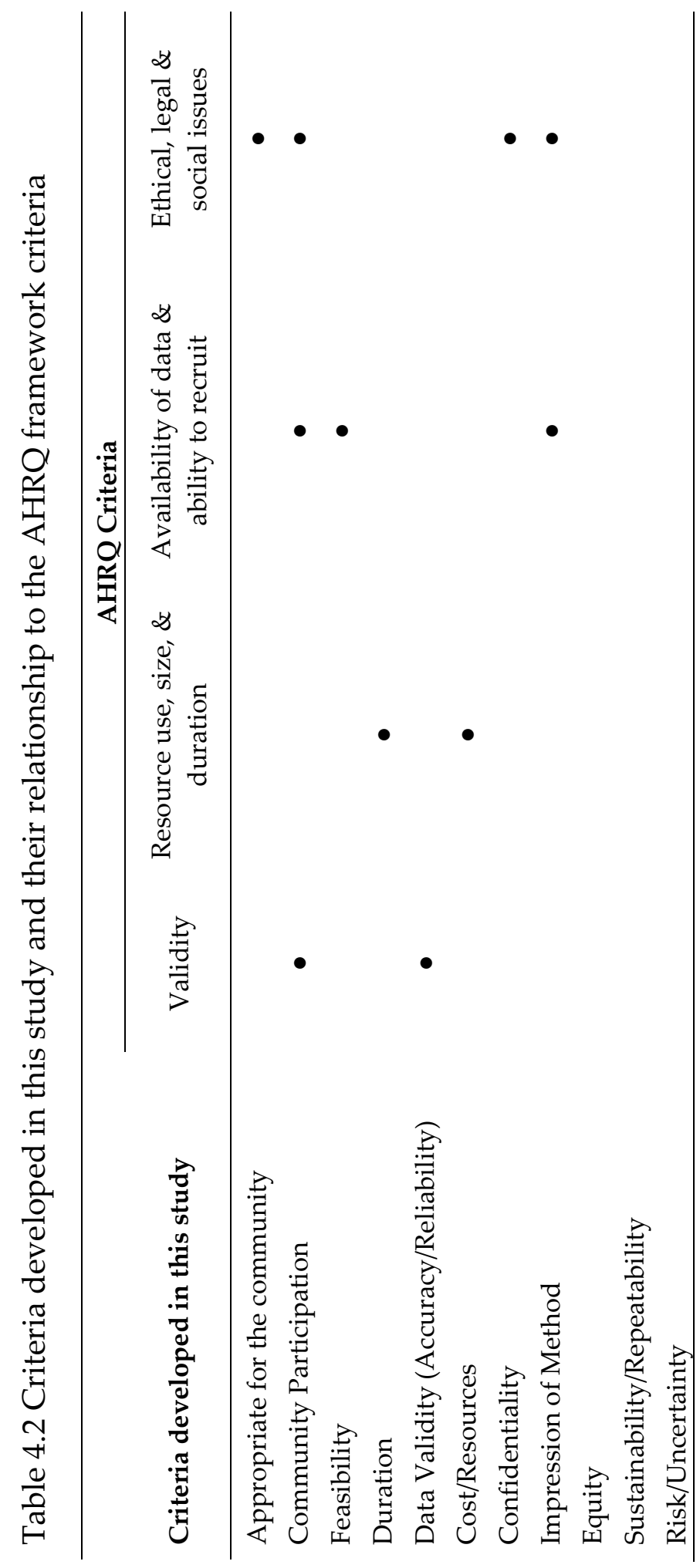




\section{Community participation}

The importance of the participation of the community in size estimation studies was reiterated by most of the key informants $(n=13)$, including three participants from the government, two participants from NGOs and research institutes, and eight participants from the community of people at risk of HIV. They cited examples of recruiting peer-educators to help identify hotspots, building relationships with establishment workers to allow access to interview the at-risk populations, and working with local authorities to triangulate data for improved accuracy. The latter involvement of local authorities also helps to reassure participants in size estimation studies about the legality of the study, which was cited by one key informant as a common concern particularly in provinces. Community participation is also a means to ensure learning for the community, which is linked to the sustainability and repeatability and long-term costeffectiveness of the study methods. Five key informants, all of whom came from the community of people at risk of HIV, considered community learning as an important aim of community participation.

It is not just the relations and networks that matter in population size estimation studies, but also the reputation of the investigators. The key informants told us that when participants in studies trusted the investigators, they were more forthcoming and honest, and complete in their disclosure. Trusted members of the community are privileged to receive unadulterated information from their peers, which helps to generate accurate and reliable size estimates.

Communities of key populations, like any social networks, are not symmetrically connected, and members of the community vary in the strength of their ties. Local knowledge about the "sociometric stars" (individuals whose high regard among 
their peers enables them to recruit their peers (Wejnert \& Heckathorn, 2008)) and hidden community members increases the speed of reaching the desired number of participants in size estimation studies, as well as the reach of the study to a diverse range of community members. One key informant however, refuted the notion of community participation to improve recruitability, saying that "hidden populations never take part in community activities", so the involvement of community peers and social networks do not amount to universal recruitability.

\section{Confidentiality}

While nine key informants considered confidentiality as a criterion in the selection of methods for size estimation, most of those key informants were at-risk population community members $(n=6)$. The level of confidentiality they mentioned ranged from total anonymity to discretion. One key informant contextualized the confidentiality issue in relation to the level of stigma in the region where studies are conducted: "In Ho Chi Minh City, confidentiality is not a big deal but in nonurban areas where stigma is high, people might not want to leave phone numbers." Another key informant said that providing personal information had also to do with the self-stigma: "ones who are 'closed status' will not provide it", referring to those who are not open with their friends and family about their homosexuality, HIV status, or other socially stigmatized status. Key informants familiar with self-administered surveys noted that the privacy and confidentiality inherent in self-administered surveys improved the data accuracy by reducing social desirability bias that are more prevalent in face-to-face surveys.

Contrary to the sentiments that confidentiality should be a criterion, one key informant experienced in implementing size estimation studies, noted that "people report to local government authorities if they are asked to take part in a survey. 
They want reassurances". In other words, attempts to keep the survey confidential are futile, because people are afraid they are doing something illegal. This implies that confidential surveys may conflict with the criterion of feasibility (see below).

\section{Cost/Resources}

More than one half of the key informants had something to say about costs and resources required for population size estimation $(n=9)$, including five participants from the government, three participants from NGOs and research institutes, and one participant from the community of people at risk of HIV. The views on cost/resources varied widely among participants. One thought that cost should be the last criterion, while another placed it as the main criterion, preferring a lowcost method. One key informant addressed the concerns about cost by suggesting: "method should have flexibility to keep costs down by using volunteers". Another key informant refuted the idea, giving an example of their experience using student volunteers: “One year we used students to do mapping. They could not identify the correct location of drug users or sex workers. Where there were drug users, they said no. Where there were many females but it turns out they were not female sex workers". The key informant went on to propose enlisting the help of community members, drug users turned peer-educators, in the size estimation studies. Another key informant further qualified this proposal by suggesting that "quality and number of staff with adequate capacity" should be considered when estimating resource needs.

\section{Data validity}

Data validity, expressed as reliability and accuracy of data, was the most frequently cited criterion in considering the methods of population size estimation $(n=13)$. There was little divergence in terms of how accurate the generated data 
should be. Several key informants talked about "acceptable", "adequate", and "reasonable" data validity $(n=3)$. One key informant said, "70 to 80 percent accuracy is good enough; doesn't need to be 100 percent accurate". Another key informant affirmed this opinion, saying "just get closer to the truth".

There was more divergence in how important the data validity criterion is vis-à-vis other criteria. Some key informants considered it a sub-criterion of cost/resources $(n=4)$. "Given available resources, we should aim to produce good results. If resources are limited, we should aim to produce adequate results" one key informant explained. Another key informant stated that data accuracy depended on the skills of investigators in the size estimation as well as the involvement of the community.

\section{Duration}

Half of the key informants expressed some preferences - either longer or shorter for the duration of size estimation studies $(n=8)$. One key informant reasoned that "time required for implementation should be short, so that the estimates can be repeated often for update of the data", suggesting that repeating the exercise over and over again will reinforce the reliability of the data. A key informant who had also been a participant in a recent size estimation study of men-having-sex-withmen, had a different perspective: "[they] like the quick-to-fill surveys, though it is probably skipping many additional questions that would improve reliability". Both key informants ultimately agreed that reliability was the desired outcome, despite duration of the study.

Other differences on duration were around the accuracy of short duration studies. "Time for census should be increased to identify if a person is from [this province] 
or here temporarily" said one key informant, while another pointed out: "seasonal nature of sex work makes some methods inaccurate, because of extended time required for the method". However, they conceded that longer duration studies came at higher cost as well. A critical perspective of duration as a criterion came from a key informant who considered it "a sub-criterion after considering the urgency of the data needed."

\section{Equity}

A few key informants made references to the differences in the applicability of the population size estimation methods in different geographical areas: rural versus urban areas $(n=4)$. The most frequent reasons included geographic grouping of key populations in hotspots, more prevalent use of Internet and mobile devices in urban areas, and better roads and access in cities than rural mountainous provinces. Not all opinions however favored urban environments, with one key informant saying: "rural studies are easier; people are more likely to answer honestly".

Two key informants invoked the differences in the methods' ability to work for different key populations. Methods that rely on recognition or identification of hotspots were questioned for particular key populations: "Female sex workers are easier to recognize, gather in hotspots; men who have sex with men use social networks, so reach is less costly" one key informant stated.

Age of people at risk of HIV also factored into the size estimation methods' equitable application to all populations. Community activism is relatively new in Viet Nam, so younger gay men are more involved with the LGBT community and 
therefore it is easier to recruit their help in size estimation studies when the study aims are aligned with community aims.

\section{Feasibility}

All factors that are external to the methods of size estimation, such as the environment and history, which affect the decision to select one method over another are considered issues of feasibility. Two recurrent external factors mentioned in the interviews were 'willingness to participate' and 'structures in place'. These factors were merged to develop the criterion of feasibility. Majority of key informants mentioned this criterion $(n=12)$, including all at-risk population community members who were interviewed $(n=8)$.

For two key informants, the notion of 'willingness to participate' stemmed from the at-risk population's sense of community and civil duty towards that community. That is, the stronger the sense of community among the members, the more willing they would be to participate in the size estimation studies, thus making the study more feasible (see criterion on Community Participation). Key informants also noted 'convenience for participants' as a factor in determining the willingness to participate $(n=4)$. This convenience was both in terms of how easy the questions in the surveys would be to answer, but also how convenient the process would be for participation. An online survey for example would be easier to organize for participants and investigators, than a face-to-face interview that would require organizing a convenient time and place for both.

Key informants mentioned that 'stigmatized subjects' and 'survey fatigue' are two deterrents to the participation in size estimation mentioned $(n=4)$. In the former case, one key informant said: "if the theme is sensitive and involves stigma, it is 
difficult to do". In the latter case, survey fatigue is a result of a long history and large volume of surveys and surveillance activities - often without the involvement of the community - that has diminished the ability of new surveys to recruit participants, and therefore diminishes the feasibility of future size estimation studies.

Key informants frequently talked about 'structures in place' that make size estimation studies more feasible $(n=8)$. Key structures elicited in the interviews included technologies, like Internet and mobile network access, to facilitate surveys. Other structures in place included key population gathering hotspots, social networks of key populations, and physical infrastructure such as roads to reach rural mountainous provinces. Experienced investigators were also noted as making a positive contribution to the feasibility of study methods. In the absence of these structures in place, the choice of methods for size estimation diminishes along with the feasibility of the study methods.

\section{Impression of method}

One of the themes that developed in the interviews, and the third most frequently cited criterion, was around the impressions that the key informants held about the method of size estimation, and how that impression affected their choice of method $(n=10)$. In reference to various methods, the key informants used phrases like "sense of seriousness", "seems exclusive", "seems rigorous", "more professional", "have confidence in", "state-of-art", and "novel". In probing the key informants, one said "people like things related to technology", in reference to novel methods of using social media for size estimation. The impression of "exclusiveness" was explained by another key informants as being created by 
disqualifying some respondents: "I was surprised by the limited number of invitees", one said, "not like the typical poll created online".

The most compelling explanation came from a key informant who explained the criterion from the perspective of decision-makers: "simple methods are often seen with skepticism, whereas more complicated methods carry more weight. Perception of people about the method matters. Sometimes people prefer more complicated methods, because it sounds more scientific and so it must produce better results. A method that involves simple counting might be suspected to be too easy to be true. Sometimes, in order to get buy-in, we may need to rely on more complicated methods."

The impression that the key informants had of a method was clearly a criterion for the selection of that method. Novel methods were positively considered by the key informants. This novelty of method addresses survey fatigue, increases interest and recruitment of participants, and improves acceptance of results by stakeholders. However, two key informants who had been involved in recent size estimations as investigators recalled "the novelty of the method made it a painful process", and felt "anxious" about getting results. Novel methods also lacked the historical data to validate the reliability of their results.

\section{Repeatability}

A criterion that is closely linked to, but distinct from the community participation and cost criteria in choice of population size estimation is the repeatability of the method. Only one key informant mentioned this criterion. It speaks about sustainability and the long-term cost-effectiveness of a method as the community 
learns how it works and applies it using volunteers in the community. As the key informant put it, a method that "people can learn and do it later".

\section{Risk}

An important but seldom mentioned criterion was the dependence of the methods on uncontrollable factors $(n=1)$. This theme emerged from a conversation with one key informant who was involved in a recent respondent driven sampling survey, where referrals were trickling in too slowly and jeopardizing the validity of the results, and also increasing the overall cost of running the study. Methods of population size estimation that use respondent driven sampling carry more uncertainty because they depend on people's willingness to refer. This uncertainty in the methods' ability to produce the required results was given a thematic label of risk.

\subsection{Discussion}

We explored the perspectives of multiple stakeholders in Viet Nam who were previously involved in population size estimation studies, on criteria relevant to selecting methods of population size estimation for surveillance of HIV epidemic, and the extent to which these criteria agree with the AHRQ framework for Considering Study Designs for Future Research Needs (Carey et al., 2012). Our findings are consistent with the AHRQ framework, but our work further clarifies the dimensions of this framework when applied to population size estimation methods, and extends it to include three newly identified criteria: repeatability, risk, and equity. The latter equity criterion was defined in terms of methods that are appropriate for different age groups, at-risk populations, and urban/rural settings. The addition of these criteria to the AHRQ framework will increase its breadth and 
relevance to the prioritization of methods for estimation of size of populations at risk of HIV.

In addition to the new criteria identified, it is important to note that some other criteria would have been missed if the study did not include a diverse group of stakeholders. While all key informants from the government, NGOs and research institutes considered cost an important criterion, only one key informant from the community of people at risk of HIV thought this criterion was important. Conversely, community participation was considered a criterion by the majority of key informants, but no key informants from the government, NGOs and research institutes saw it as an opportunity for community learning. Our findings illustrate the dichotomy of views of stakeholders on criteria for prioritization of methods of size estimation, and underlines the importance of an inclusive and interactive process that considers the opinion of technical experts, health managers, but also the community that is the beneficiary of the evidence-based services (Grin et al., 1997; Ham \& Coulter, 2001; Vuorenkoski, Toiviainen, \& Hemminki, 2008). An important implication of this finding at the national level is the need for inclusive decision-making that involves the community. While participation of community members in strategic planning of the HIV response in Viet Nam is affirmed (National Committee for AIDS Drug and Prostitution Prevention and Control, 2012; Viet Nam Administration of HIV/AIDS Control, 2007), their participation in technical and scientific decisions like those of population size estimation methods must also be supported. This "democratization of expertise" may well require investments in technical literacy of community based organizations to strengthen their role in decision-making or grass-roots movements for community-driven policies in research, science and technology (Beeker, Guenther-Grey, \& Raj, 1998; Tickner \& Wright, 2003). 
Among criteria elicited by multiple key informants from diverse groups, perspectives of how a criterion is defined sometimes diverged significantly. Feasibility, for example, is a criterion that is often elicited in research prioritization (McGregor et al., 2014; Montorzi et al., 2010; Okello et al., 2000). In our conversations, key informants from the government, NGOs and research institutes defined feasibility in terms of structural enablers in place to support the method, such as mobile telephone technology, roads to get to remote villages, and experienced investigators. We call this the systems perspective of the criteria. Key informants from the community of people at risk of HIV, clarified feasibility in terms of the individual recruits' willingness to participate in the size estimation studies - due to convenience of participation, interest in the novelty of the method and the learning opportunity, and a sense of community or civil duty to their community. We call this perspective the community perspective of the criteria. Another instance in our study where the community and system perspectives are evident, is in the discussions around duration. One key informant spoke about duration from the perspective of an individual survey taker (community perspective), while another took the perspective of the entire duration of a size estimation study (system perspective). Our study points out the importance of this dual perspective to help decision-makers derive a more complete and legitimate definition of the criteria.

Moreover, the findings suggest that feasibility of some methods may depend as much on the systems and structures in place, as it does on the strength of ties within the community of participants who are the subjects of the study. In line with the recommendations of Johnston et al. (Johnston, Whitehead, Simic-Lawson, \& Kendall, 2010), our findings point out a specific need for better evidence about the strength of ties within the community, as an indicator of their willingness to participate in population size estimation studies. The strength of ties can be 
measured for example by conducting a survey in the community, recruiting participants through RDS, and asking respondents to grade their relationship with the person who referred them using profiles of relationships developed by Spencer and Pahl (Spencer \& Pahl, 2006) or using Dunbar's theoretical boundaries of social contacts (Dunbar, 2011).

Where there was agreement on the definition of criteria, key informants sometimes differed in how important they considered one criterion vis-à-vis others. In prioritization frameworks, these relative differences are called the weight of the criteria (Marsh et al., 2016; Marsh, Lanitis, Neasham, Orfanos, \& Caro, 2014). Another crosscutting theme that emerges in reviewing the criteria elicited in this study is that there were differences among the key informants about the direction of some criteria. These differences were sometimes considerably varying, with some key informants seeing a criterion as a positive factor for selecting a method, and others seeing it in a negative light. Criteria that exhibited these differences in weight and direction include confidentiality, cost/resources, data validity, duration, equity and impressions of method. The weight and direction of criteria affect the priorities in methods of population size estimation when the criteria are applied. A number of structured procedures exist to quantifying the criteria weights and directions of the criteria. Such procedures include discrete choice experiments, conjoint analysis, ranking and rating of criteria (Marsh et al., 2016, 2014). These procedures would be an important addition to the AHRQ framework to prioritize methods of population size estimation.

The findings above on missing criteria, inclusive participation, community perspectives and conflicting weight and direction of criteria, provide insights that help us improve the AHRQ framework in its application to the prioritization of population size estimation methods. These findings underline the importance of 
inclusion of diverse group of stakeholders, particularly the community of people at risk of HIV. These findings and recommendations are also in line with the expectations of the authors of the AHRQ framework for it to be refined and contextualized in the future (Carey et al., 2012).

To our knowledge, at the time of this study there is no known application of the AHRQ framework in developing country settings or to HIV surveillance. This study furthers our understanding of methodological issues that may be faced in applying the framework. Comparison of the study findings in Viet Nam, to best practices found in literature, allows us to provide a number of suggestions to clarify the role of stakeholders in the priority setting process:

First, our results showed a number of potential conflicts and dependencies between criteria identified. For example, two key informants in our study had different definitions of the duration criterion, but ultimately agreed that reliability was the desired outcome. Youngkong et al., who conducted a systematic review of health care priority setting in low-income countries, posit that differences in definitions of criteria may be dependent on culture and perspectives of the stakeholders (Youngkong et al., 2009). They predict that in joint discussions with relevant stakeholders a more suitable set of criteria may be obtained. Guidance on multi-criteria decision-making recommends focusing the group discussion on organizing criteria into a hierarchical structure, and combining criteria when there is potential redundancy and decomposing criteria when alternative definitions of criteria are elicited (Mabin \& Beattie, 2006). This process of representing the decision analysis jointly is believed to have an indirect value in raising consciousness about the root of any conflict (Goodwin \& Wright, 2004). 
Second, our results showed potentially different weight and directions assigned to the criteria by a group of stakeholders. Kerr and Tindale have discussed the use of a number of approaches to group decision-making (Kerr \& Tindale, 2004). Perez et al present use of fuzzy set theory to model and deal with vague or imprecise options, alternatives, and opinions of several decision-makers (Pérez, Wikström, Mezei, Carlsson, \& Herrera-Viedma, 2013). Shukla and Auriel suggest a framework for conducting criteria weight analysis under multi-stakeholder scenarios, but with an emphasis on transparency, avoidance of conflicts, low cognitive load, and taking into account multiple decision-makers with different perception of criteria (Shukla, Auriol, \& Hipel, 2016). It is the latter approach that we recommend for the management of diverse definitions, weight and directions of criteria when a wider group of decision-makers, including community members, are consulted in decisions on population size estimation methods.

The primary aim of this study, like other qualitative research, is to provide a rich, contextual understanding and not to generalize results, so representative samplings is not as important as the ability of the selected participants to provide their diverse perspectives (Horsburgh, 2003; Leung, 2015; Polit \& Beck, 2010). Nevertheless, the depth and coverage of those perspectives on population size estimation methods is limited in our study by the time allowed with few key informants in few locations, and how the key informants were selected. We tried to overcome the limitation in the external validity of our study by employing the four strategies recommended by Sharan Merriam (Merriam, 1995). These include (1) providing enough details in our study so that readers can determine how closely their situation matches it, (2) using multiple sites to allow for application to a greater range of similar situations, (3) comparing the specific criteria in this study to the broader criteria of health research in the AHRQ framework, and (4) 
sampling within the key informants to ensure representation of the relevant three stakeholder groups.

The AHRQ framework was selected for this study because it provided us with a standardize terminology, fair process, and basic set of criteria to compare against our findings in Viet Nam. Although there is a lack of application of the framework outside of the United States, an aim of this study was precisely the applicability and relevance of this framework to decisions on methods of size estimation for key populations at risk of HIV in Viet Nam.

Another limitation of this study was that no focus group discussions were conducted. Without a debate to test the strength of opinions of key informants on any particular subject, vis-à-vis their peers, we cannot be certain how strongly individuals believe in their opinions. On the other hand, the in-depth interview format did allow some valid, but less popular, points of view to be exposed. The selection of key informants was through introductions from the seed key informants. It is possible that key informants refer individuals similar to themselves in perspectives and experience. However, private interviews with the key informants, and conducting interviews in two separate cities, help to ensure the independence and trustworthiness of the results.

We intended to identify and interview a diversity of key informants, both geographically and also in their representation of key stakeholder groups. We succeeded in recruiting equal number of participants from Ho Chi Minh City and Vinh City. In terms of representation of the three key stakeholder groups, there were fewer representatives from NGOs and research institutes, and greater representation from the community members, due to their availability at the time of the interviews. This could have led to some skewing of relevant criteria in our 
results toward the community perspective. However, the comparison of the criteria elicited in this study to the criteria in the AHRQ framework gives some external validation of the results.

In the analysis of the interviews all three investigators were involved in the categorization and thematic grouping of codes. However, only one investigator codified the transcripts. While multiple coders would have added rigor and richness to the results, it would have required far more time to review the transcripts and reconcile the codes generated. Having one investigator coding the transcripts also allowed a more uniform definition of the codes to be applied across all transcripts.

\subsection{Conclusion}

Findings of this study suggest that exclusion of community members from decision-making around key at-risk population size estimation methods in Viet Nam may be contributing to reduced validity, use, and efficiency in evidence generated from these types of surveillance activity. A wider group of decisionmakers, including community members among others, may introduce diverse definitions, weight and direction of criteria. Based on these findings, and best practices in the decision-making literature we developed a number of recommendations to update and contextualize the AHRQ framework to decisions around HIV surveillance and population size estimation in Viet Nam.

For Viet Nam, we think the AHRQ framework does not have all the criteria that are relevant to stakeholders, and these criteria should be added and considered in future studies. We also suggest using the dual "systems perspective" and "community perspective" help clarify the different definitions of common criteria. 
When these two perspectives are conflicting, we recommend a process of organizing criteria into a hierarchical structure jointly with relevant stakeholders, and conducting a criteria weight analysis under a multi-stakeholder scenario.

The lessons from Viet Nam may not apply to every country with a transitioning economy. And the lessons from the HIV response may not apply to every emerging epidemic. However, some of the principles of fair decision-making, value of community participations in decision-making and the expected challenges faced, merit considering in every situation. 


\section{Chapter 5: Criteria for Prioritization of HIV Programs in Viet Nam: A Discrete Choice Experiment}

This chapter draws upon:

Safarnejad A, Pavlova M, Son VH, Phuong HL, Groot W. Criteria for prioritization of HIV programs in Viet Nam: a discrete choice experiment. Submitted for publication. 


\section{Abstract}

With the decline in funding for Viet Nam's HIV response, there is a need for prioritizing a package of interventions in an HIV programs, although there is a gap in the research on the relative importance of multiple criteria for that prioritization. This study elicits preferences and the trade-offs made between different HIV programs by stakeholders and decision-makers in Viet Nam, while paying attention to how social and professional characteristics shape their preferences.

This study uses self-explicated ranking and discrete choice experiments to determine the relative importance of five criteria - effectiveness, feasibility, costeffectiveness, rate of investment and prevention/treatment investment ratio - when stakeholders evaluate and select hypothetical HIV programs.

Our findings show that the feasibility criterion is more important to participants when they choose an HIV program, than other criteria. The participant's professional characteristics have a significant effect on the importance of some criteria. In the self-explicated ranking effectiveness ranks highest and the costeffectiveness criterion ranks low in importance across all groups.

This study has shown that the preferred HIV program in Viet Nam is feasible, front-loaded for sustainability, has a higher proportion of investment on prevention, saves more lives and prevents more infections. Similarities in government and civil society rankings of criteria are grounds for future policy dialogues between stakeholders. 


\subsection{Introduction}

The first case of HIV infection in Viet Nam was reported in December 1990 in Ho Chi Minh City. By December 2003, 76,180 infections were reported in Viet Nam and 6,550 people had died of AIDS related causes (General Statistical Office at the National Institute of Hygiene and Epidemiology/Vietnam and ORC Macro, 2006; The Government of the Socialist Republic of Viet Nam, 2004). By December 2015, there were 255,000 people living with HIV and over 128,000 people had died from AIDS-related illnesses since the start of the epidemic (UNAIDS, 2015).

Viet Nam's HIV epidemic is concentrated among three key population groups defined by risk behaviors and a high prevalence of HIV: people who inject drugs, men who have sex with men and female sex workers (Vietnam Authority of HIV/AIDS Control, 2013). The main route of transmission is through injecting drugs followed by sexual transmission. By 2015, the estimated number of new infections had decreased by 50 percent from the peak of the epidemic in 2003, thanks to prevention initiatives for key populations, including the provision of clean needles and syringes, provision of condoms, methadone maintenance therapy, and antiretroviral treatment (UNAIDS, 2015; Viet Nam Ministry of Health, 2014).

International donors have provided substantial support to Viet Nam's HIV response. In 2005, The U.S. President's Emergency Plan for AIDS Relief (PEPFAR), Asian Development Bank, World Bank, UK Department for International Development (DFID), The Global Fund to fight AIDS, Tuberculosis and Malaria (GFATM), and Australian Agency for International Development were financing a significant portion of the HIV programs (Vietnam Ministry of Planning and 
Investment, 2015). Viet Nam's recent reclassification as a lower middle-income country, introduced a challenge in financing of the HIV response (World Bank, 2013) because most donors provide more official development assistance to lower income countries than other income groups (United Nations Development Programme \& Bureau for Development Policy, 2011). By 2015, only GFATM and PEPFAR remained in Viet Nam to provide funding for the HIV response, including funding $95 \%$ of the costs of Anti-retroviral Treatments (Health Finance \& Governance, 2014). The national HIV program faced sustainability issues due to the substantial decline in external donor funding commitments beyond 2017 (Vietnam Ministry of Health, 2016). The rapid phase out of donors has alarmed the government of Viet Nam. Therefore, the Deputy Prime Minister has called on the international donor community to give the country more time to transition to domestic funding of the HIV response, including use of social health insurance for curative care (Minh, 2016).

Global shortfalls in funding for the HIV response make it unlikely that the withdrawal of international funding will slow down in Viet Nam. The 2016 report of the Kaiser Family Foundation and UNAIDS indicated that donor funding to support the HIV response efforts in low- and middle-income countries, had declined for the first time in five years (KFF \& UNAIDS, 2016). "Donors faced many competing funding demands, including humanitarian emergencies and the refugee crisis, all against a backdrop of fiscal austerity in a number of countries", explained Jen Kates, Kaiser Family Foundation Vice President and Director of Global Health and HIV Policy (UNAIDS, 2016f).

In 2012, after a decade of financial support to Viet Nam's HIV response, the World Bank and DFID ended their funding. Their recommendation for Viet Nam before exiting was to refocus the government funding of HIV prevention programs on 
provinces in the country based on epidemiological impact, infrastructure, and ability of communities to mobilize resources (Zhang et al., 2012). In 2014, Viet Nam's Ministry of Health, with support from UNAIDS, developed the Investment Case, which identified priorities and the most effective approaches for the National HIV response (Viet Nam Ministry of Health, 2014). Although commendable, the Investment Case limited the prioritization criteria to effectiveness and costeffectiveness. And while other criteria, like sustainability, were mentioned as principles, there was no explicit use of other criteria for priority setting. Faced with shrinking donor funds, future prioritization initiatives in Viet Nam may lean further towards prioritizing the cost-effectiveness of the HIV program package, while neglecting other relevant criteria.

Given the limited funding, there is a growing interest in generating evidence on the criteria to guide priority setting in the HIV response (Baltussen, Youngkong, Paolucci, \& Niessen, 2010; Hogan, 2005; Kabaniha, 2014; Leelahavarong et al., 2011; Tromp, Prawiranegara, Siregar, Jansen, \& Baltussen, 2016; Youngkong, Baltussen, Tantivess, Koolman, \& Teerawattananon, 2010). A number of studies have considered multiple criteria explicitly to prioritize specific prevention interventions (Leelahavarong et al., 2011; Newman, Cameron, Roungprakhon, Tepjan, \& Scarpa, 2016; Verguet, 2013) or HIV treatment (Baltussen et al., 2013; Walensky et al., 2010). In Indonesia and Pakistan, a broad set of HIV interventions in the national HIV response were considered, and stakeholders were involved in self-explicating the importance of criteria for priority setting (Husain, Kadir, \& Fatmi, 2007; Tromp, Prawiranegara, Subhan Riparev, et al., 2015). A Thai study used more rigorous experimental methods to rate criteria that guide priority setting, involving decision-makers as well as stakeholders living with or at higher risk of HIV, thereby reducing the bias in self-reported importance of criteria (Youngkong et al., 2010). The design of the Thai study considered prioritization of targeted 
interventions rather than the program package of interventions. In a number of the mentioned studies, diverse stakeholder groups were convened to consider one criterion for prioritizing HIV interventions, while other studies convened a limited group to consider multiple criteria. The aforementioned studies were limited in terms of a narrow focus on a limited set of interventions, a lack of involvement of key stakeholders, or use of less rigorous study designs. These limitations collectively have left a gap in the research on the relative importance of multiple criteria for prioritizing a package of interventions.

This study elicits preferences and the trade-offs made between different HIV programs by relevant stakeholders and decision-makers in Viet Nam. In other words, given several criteria for deciding on a HIV program, how much of one criterion are they willing to give up for improvements in another criterion. We also pay attention to how differences in social and professional characteristics of stakeholders and their agency affiliations shape preferences for HIV program criteria in Viet Nam. This study is innovative in its use of discrete choice experiments (DCE) and self-explicated ranking to establish the relative importance of a set of criteria for prioritizing Viet Nam's HIV response.

DCE are based on well-tested theories that provide an explanation of choice behavior (Louviere, Flynn, \& Carson, 2010). These experiments place individuals in scenarios where they have to make a choice between options presented to them. In that decision-making process, the individual considers the criteria that define the options, and the trade-off in criteria they are going to make in choosing one option over another option. Data from the individual choices can then be used to quantify the relative importance of the criteria. DCE have been used extensively to examine preferences and priorities in health care [e.g., (Green \& Gerard, 2009; Larson et al., 
2015; Luyten, Kessels, Goos, \& Beutels, 2015; Mirelman et al., 2012; van de Schoot, Pavlova, Atanasova, \& Groot, 2017)].

This study contributes to Viet Nam's HIV policy-making by clarifying what criteria are being considered in prioritizing the programs in the National HIV response, and how different stakeholders view the importance of those criteria. Other countries can adopt this transparent and accountable process during their national HIV planning process and in prioritizing their HIV funding proposals to donors.

\subsection{Methods}

This study used two methods to elicit stakeholders' preferences for and choices of HIV programs. The first method was a straightforward self-explicated ranking of criteria. The second method used the DCE method to determine the relative importance of criteria to stakeholders when they evaluate and select HIV programs.

DCE is a type of hypothetical experiment that is widely used in the health field to quantify preferences. The experiment imitates a situation when a stakeholder must make a choice between two or more options. Each option has the same set of attributes as the other option but the values of these attributes are varied to make the option different from the other. In this study, the DCE attributes are referred to as "criteria", which is the common term used in priority setting and operations research. The variance of the criteria was fixed to two levels. To reduce the cognitive burden required by the respondents (stakeholders and decision-makers), the number of choice scenarios presented to them was minimized using orthogonal arrays. Each choice scenario contained two HIV programs. During the experiment, 
the choice scenarios were presented to the respondents consecutively, and the respondents were asked to take their time to select the HIV program they preferred most in each choice scenario.

\section{Selection of criteria and levels}

The most frequently used criteria identified in a systematic review of literature served as a basis for the DCE in this study (Chapter 2). The full set of 18 criteria in the systematic review were reviewed with HIV experts working in Viet Nam to assess their relevance to the country's HIV epidemic and response. Based on that assessment, five criteria of a HIV program were selected: effectiveness, feasibility, cost-effectiveness, rate of investment and prevention/treatment investment ratio. The first three of these criteria were characterized as program outcomes. The last two criteria were characterized as program inputs.

All criteria had dichotomous levels. These criteria levels were generated based on investment case scenarios developed for Viet Nam in 2014 (Viet Nam Ministry of Health, 2014). The investment case scenarios were developed using the AIDS Epidemic Model (AEM). AEM is a standard estimation and projection tool used in modeling the epidemic in countries with concentrated epidemics. The AEM uses input program and epidemiological data to estimate the future impact of the proposed policies and program coverage levels, as well as the size of the investment required. One of the assumptions that was used in the selection of criteria levels, was that the AEM projections went until 2030, when international and national goals are to be met. Additionally, it was assumed in the AEM estimations that funding would be capped to the current level of spending, and should not be expected to increase beyond current levels. Table 5.1 presents all criteria, their levels and coding for the analysis. 


\section{Study instrument, DCE design and DCE validity}

Prior to the DCE, the questionnaire presented an operational definition of each criterion, and asked respondents to manually rank the criteria according to the importance they attached to each of the criteria. This step allowed the comparison of self-explicated ranking with the DCE weighted ordering of the criteria. It also allowed a common understanding of the criteria by the participants prior to starting the DCE.

To limit the number of combinations of scenarios and avoid information overload by participants in the DCE, criteria were limited to five with two levels each (see Table 5.1), which resulted in a total of $32\left(2^{5}\right)$ possible profiles. A subset of those 32 profiles was chosen on the basis of a fractional experimental designs library of orthogonal arrays (Hedayat, Sloane, \& Stufken, 1999). The fractional factorial design included a subset of eight profiles that allowed the estimation of all main effects. The construction of eight profiles and related coding is presented in Table 5.1.

One of the eight profiles defined by the fractional factorial design was selected as the basic profile, and the rest were used as alternative profiles. The basic profile was selected to create realistic and challenging decision situations for participants in the experiment. Then seven choice scenarios were created where respondents were asked to choose between the basic profile and one of the alternative profiles based on their preference for the criteria for prioritizing the HIV response. A sample choice scenario from the DCE is presented in Figure 5.1. This DCE design minimized the number of comparisons (choice scenarios) respondents had to make while giving a reasonable estimation of the main effects. 


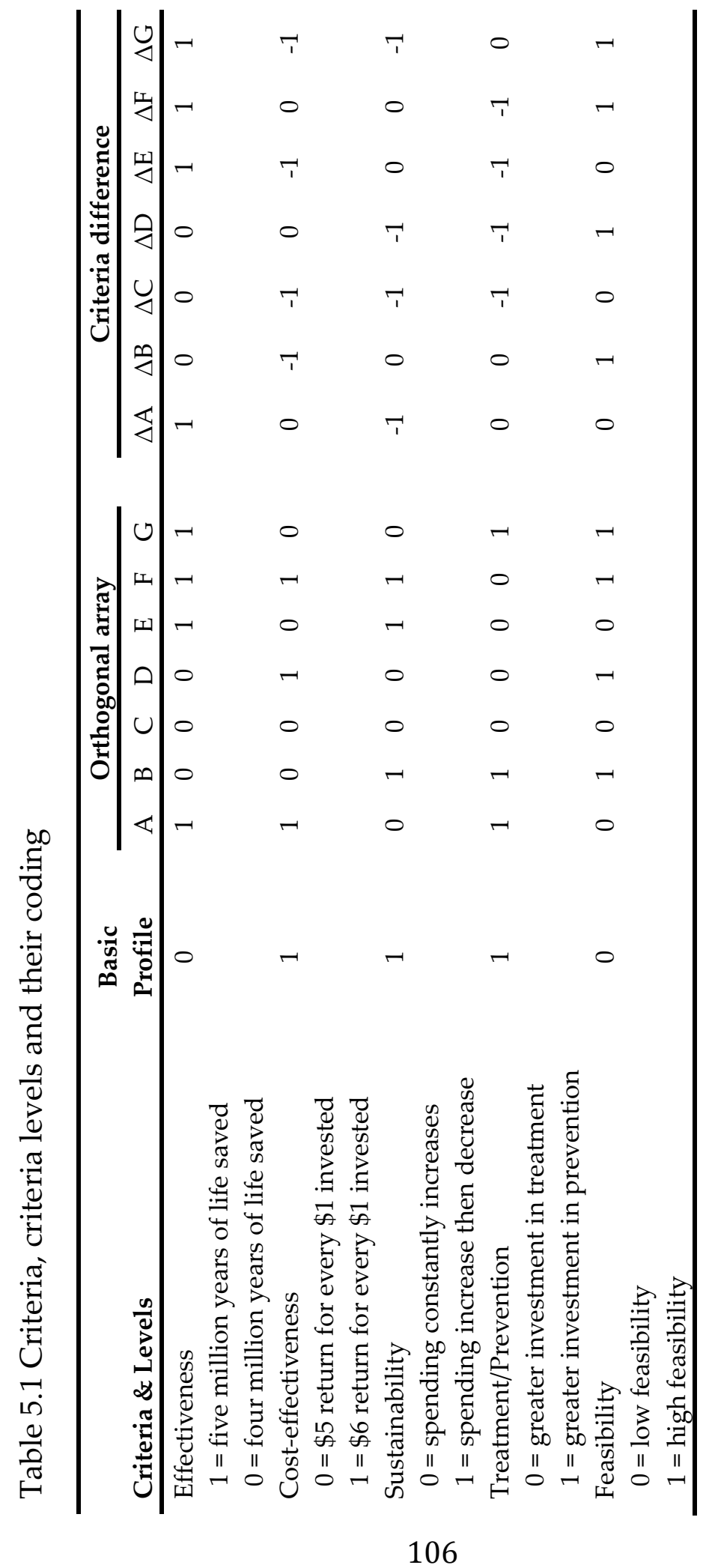


The survey that presented the DCE also collected information on the respondent's social and professional characteristics. These data were used to assess how individual factors influence decision-maker's stated preferences. The assessed characteristics included age, sex, nationality, professional experience, and engagement in decision-making in Viet Nam's HIV response. The complete survey instrument is included in Appendix I.

Figure 5.1 A sample choice scenario in the discrete choice experiment

\begin{tabular}{|c|c|c|c|c|}
\hline Effectiveness & $\begin{array}{c}\text { Cost- } \\
\text { Effectiveness }\end{array}$ & $\begin{array}{c}\text { Rate of investment * } \\
\text { (in Million USD) }\end{array}$ & $\begin{array}{c}\text { Resource Allocation to } \\
\text { Prevention vs. Treatment }\end{array}$ & $\begin{array}{c}\text { Feasibility to } \\
\text { Implement Program }\end{array}$ \\
\hline
\end{tabular}

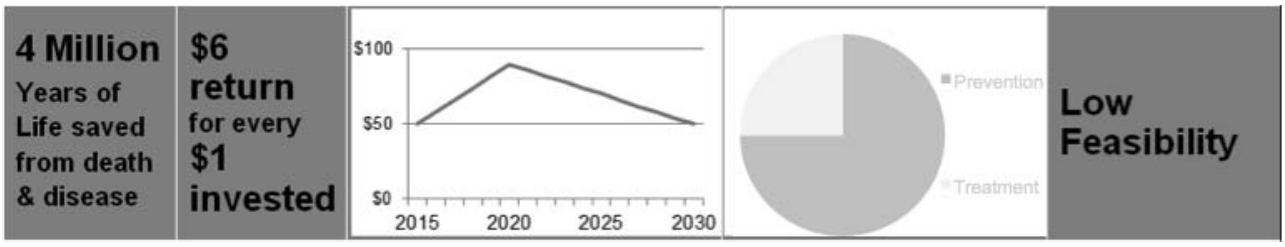

\section{Million \\ Years of \\ Life saved \\ from death \\ $\&$ disease}
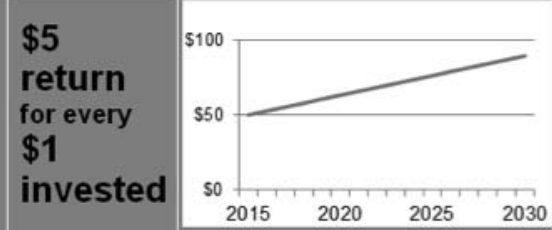

\section{High}

Feasibility

\section{Study setting and participants}

The DCE was conducted in Viet Nam, where the HIV response involves development partners, civil society, and the central and provincial state institutions. The study aimed to reach 60 participants who had been involved, or currently are involved, in the decision-making in Viet Nam's HIV response, with similar number of respondents from government, international development partners, and civil society organizations respectively. The motivation behind selecting these three groups was that they encompass the actors who have a stake in the decision-making in the HIV response. These three groups are referenced by 
the Greater Involvement of People Living with HIV principle formalized at the 1994 Paris AIDS Summit (Joint United Nations Programme on HIV/AIDS, 1999), and are also reflected in the membership structure of the Country Coordinating Mechanism of the GFATM (GFATM, 2013). The proportion of individuals in the three groups may not represent the same proportion of individuals who were involved in decision-making processes in Viet Nam at the time of the study, however. For example, the GFATM Country Coordinating Mechanism involves 7 individuals from the government, 6 from international development partners, and 11 from civil society organizations (Country Coordinating Mechanism Vietnam, 2015). The steering committee for development of the investment case scenarios involved 3 individuals from the government, 6 individuals from international development partners, and no civil society members, although the latter were involved in consultations to obtain inputs "on their priorities for the response in future" (Viet Nam Ministry of Health, 2014). The National Strategic Plan for HIV/AIDS Prevention and Control in Viet Nam was developed with active participation of the three groups, but the extent of that participation or process of prioritization has not been documented (The Government of the Socialist Republic of Viet Nam, 2004, 2006).

Participants were identified in a two-stage selective sampling of individuals knowledgeable or responsible for decision-making. In the first stage, the researchers identified seed individuals in government, civil society, and development partners. Seed individuals are initial study participants who recruit their social or professional peers (Heckathorn, 1997). In the second stage, the seed individuals initially nominated twenty individuals each within their network to potentially respond to the questionnaire. If some invited individuals did not respond or were unable to respond to the questionnaire, the seed individuals sent five new invitations to potential respondents. This process continued until the 
quota of 60 participants with similar number of respondents from the three groups was reached. The nominated individuals received a web link to anonymously respond to the survey. All eligible participants were informed of the purpose of the study and notified that they can exit the survey at any time and choose to have all their answers be deleted. Thus, informed consent to participate was provided by each respondent.

\section{Pilot of the study}

The DCE was piloted with four participants. As a result of the pilot, the ordering of choice scenarios was revised to start with the simplest task (with two differences between the two profiles) to the most difficult task (with four differences between the two profiles). The choice scenarios were also formatted to be displayed horizontally, compared to the vertical presentation customary in DCE. This allowed the respondents to give equal attention to all criteria of the profile, and reduced the dominance of the criterion on top. Finally, where graphs were used to illustrate the meaning of criteria, footnotes were added to further clarify those graphs.

\section{Survey administration, data collection and analysis procedure}

The DCE survey was administered electronically on the LimeSurvey platform using a standardized questionnaire. The participant choices were coded 0 if the basic profile was selected as the preferred profile and coded 1 if the alternative profile was selected as the preferred profile.

The data collected were inserted in Excel and cleaned of any inconsistent answers to the DCE scenarios. Two respondents provided a combination of responses that 
were categorized as inconsistent answers. Firstly, if a respondent selected a high feasibility program over a cost-effective program (choice scenario 2), then selected a program with constantly increasing costs and high proportion of spending on treatment over a cost-effective program (choice scenario 3), and then selected a low feasibility, low proportion of spending on treatment, and decreasing cost program which is highly cost-effective (choice scenario 4), that respondent's answers were deemed inconsistent. Secondly, if a respondent selected an effective program over a program with decreasing costs (choice scenario 1), then selected a highly feasible program over a cost-effective program (choice scenario 2), and then selected a costeffective and decreasing cost program over a feasible and effective program (choice scenario 7), that respondent's answer were deemed inconsistent. The DCE results of the two respondents who provided inconsistent responses were removed from the data set.

The cleaned dataset was imported into Stata for analysis. For the analysis of the DCE data, a binary logit regression with random intercepts was used. First, the main-effect model was estimated using the responses of all respondents. Then the responses were disaggregated by group membership - government, civil society, development partners - and the main effects model was estimated for each group, and the results were compared. Data from the self-explicated ranked criteria were analyzed using descriptive statistics, and ordered by the modes of the criteria ranks. The DCE results were compared to the self-explicated ordering of criteria in qualitative terms.

Social and professional characteristics may have an effect on the choices of stakeholders and decision-makers. Descriptive statistics were calculated for social and professional characteristics of the respondents. After estimating the main effects, interactions of the criteria with the social and professional characteristics of 
the respondents were added to the model. A backward stepwise regression procedure was conducted to obtain a reduced model consisting of statistically significant independent variables $(\mathrm{p}<.05)$.

\subsection{Results}

Of the 82 people invited to participate in the survey, 69 (84\%) agreed and completed the survey. Survey respondents include 31 (44\%) females and 38 (56\%) males. The majority of respondents (44\%) are between 41 and 50 years old. The majority of respondents $(76 \%)$ are Vietnamese nationals. There are nearly equal proportions of respondents from civil society, government, and development partners, with $20(29 \%), 26(38 \%)$, and 23 (33\%) representatives respectively. Most respondents (91\%) are involved in decision-making and more than one half $(65 \%)$ are responsible for decision-making. Information on all social and professional characteristics is provided in Table 5.2.

The self-explicated ranking of the criteria based on all responses shows that effectiveness is the most important criterion for respondents with $43 \%$ of respondents ranking this criterion very high. Feasibility is the next highest ranked criterion followed by sustainability, cost-effectiveness and treatment to prevention spending ratio. When responses are disaggregated by agency, effectiveness remains the most important criterion and treatment to prevention spending ratio remains the least important criterion. Most civil society respondents $(60 \%)$ and development partner respondents (48\%) select effectiveness as their most important criterion, while government respondents are equally split on feasibility and effectiveness as their top criteria (27\% and $27 \%)$. The results of the selfexplicated ranking are presented in Table 5.3. 
Results of the logit regression of the main effect model, after removing the inconsistent responses, are shown in Table 5.4. The results for the main effect model show that overall the feasibility criterion is most important to the respondents when choosing a hypothetical HIV program, followed by sustainability, treatment to prevention spending ratio, and effectiveness. The coefficient of cost-effectiveness in the main-effects model is not statistically significant. However, the main effect model does not account for the social and professional characteristics of the respondents. The influence of these characteristics can be seen in the reduced model with interactions.

Specifically, the reduced model with interactions shows that several interactions of criteria with social and professional characteristics of the respondents have a significant effect (Table 5.4). The backward stepwise regression finds six interaction terms that are statistically significant. Two interaction terms are with the effectiveness criterion. Those interactions are with respondents who are currently working, or have worked, in programming and respondents who are currently, or have been, involved in decision-making. Two other interaction terms are with the sustainability criterion. Those interactions are with respondents who are currently working, or have worked, in management and respondents who are currently, or have been, responsible for decisions. One interaction is with the criterion of the ratio of treatment to prevention spending. That interaction is with respondents who are currently working, or have worked, in management. Another interaction term is with the feasibility criterion. That interaction is with respondents who are currently, or have been, responsible for decisions. All interactions, except for the interaction of sustainability with working in management, have an overall negative effect on the ranking of their respective criteria. 


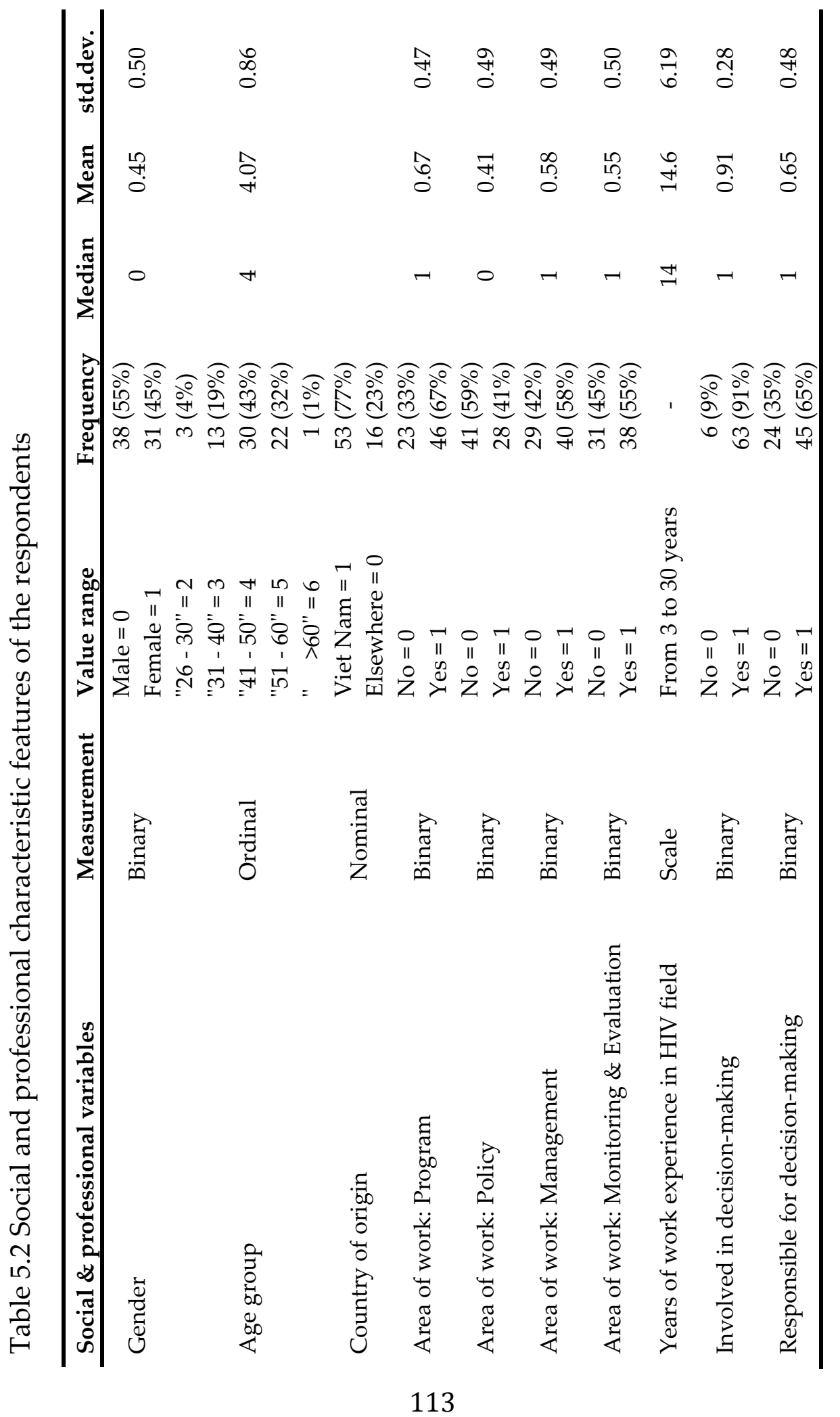


Table 5.3 Self-explicated rankings of criteria for prioritizing the HIV response

\begin{tabular}{|c|c|c|c|c|c|c|c|c|c|}
\hline \multirow[b]{2}{*}{ Criteria } & \multicolumn{3}{|c|}{$\begin{array}{l}\text { Most } \\
\text { important }\end{array}$} & \multicolumn{2}{|c|}{$\begin{array}{r}\text { Least } \\
\text { Important }\end{array}$} & \multirow[t]{2}{*}{ Mode } & \multirow[t]{2}{*}{ Median } & \multirow[t]{2}{*}{ Mean } & \multirow[t]{2}{*}{$\sigma^{2}$} \\
\hline & 1 & 2 & 3 & 4 & 5 & & & & \\
\hline \multicolumn{10}{|l|}{ All Stakeholders } \\
\hline Effectiveness & $43 \%$ & $29 \%$ & $16 \%$ & $7 \%$ & $4 \%$ & 1 & 2 & 2.0 & 1.01 \\
\hline Cost-effectiveness & $14 \%$ & $20 \%$ & $13 \%$ & $38 \%$ & $14 \%$ & 4 & 4 & 3.2 & 1.22 \\
\hline Sustainability & $14 \%$ & $19 \%$ & $28 \%$ & $28 \%$ & $12 \%$ & 3 & 3 & 3.0 & 1.13 \\
\hline Treatment/Prevention & $6 \%$ & $4 \%$ & $17 \%$ & $10 \%$ & $62 \%$ & 5 & 5 & 4.2 & 1.25 \\
\hline Feasibility & $22 \%$ & $28 \%$ & $26 \%$ & $17 \%$ & $7 \%$ & 2 & 3 & 2.6 & 1.10 \\
\hline \multicolumn{10}{|l|}{ Dvlp. Partners } \\
\hline Effectiveness & $48 \%$ & $26 \%$ & $9 \%$ & $13 \%$ & $4 \%$ & 1 & 2 & 2.0 & 1.10 \\
\hline Cost-effectiveness & $13 \%$ & $22 \%$ & $13 \%$ & $30 \%$ & $22 \%$ & 4 & 4 & 3.3 & 1.25 \\
\hline Sustainability & $4 \%$ & $26 \%$ & $26 \%$ & $30 \%$ & $13 \%$ & 4 & 3 & 3.2 & 1.02 \\
\hline Treatment/Prevention & $4 \%$ & $4 \%$ & $30 \%$ & $9 \%$ & $52 \%$ & 5 & 5 & 4.0 & 1.18 \\
\hline Feasibility & $30 \%$ & $22 \%$ & $22 \%$ & $17 \%$ & $9 \%$ & 1 & 2 & 2.5 & 1.19 \\
\hline \multicolumn{10}{|l|}{ Government } \\
\hline Effectiveness & $27 \%$ & $38 \%$ & $31 \%$ & $0 \%$ & $4 \%$ & 2 & 2 & 2.1 & 0.83 \\
\hline Cost-effectiveness & $15 \%$ & $8 \%$ & $12 \%$ & $58 \%$ & $8 \%$ & 4 & 4 & 3.3 & 1.17 \\
\hline Sustainability & $23 \%$ & $15 \%$ & $27 \%$ & $27 \%$ & $8 \%$ & 3 & 3 & 2.8 & 1.19 \\
\hline Treatment/Prevention & $8 \%$ & $4 \%$ & $8 \%$ & $8 \%$ & $73 \%$ & 5 & 5 & 4.3 & 1.30 \\
\hline Feasibility & $27 \%$ & $35 \%$ & $23 \%$ & $8 \%$ & $8 \%$ & 2 & 2 & 2.3 & 1.02 \\
\hline \multicolumn{10}{|l|}{ Civil Society } \\
\hline Effectiveness & $60 \%$ & $20 \%$ & $5 \%$ & $10 \%$ & $5 \%$ & 1 & 1 & 1.8 & 1.05 \\
\hline Cost-effectiveness & $15 \%$ & $35 \%$ & $15 \%$ & $20 \%$ & $15 \%$ & 2 & 2 & 2.8 & 1.16 \\
\hline Sustainability & $15 \%$ & $15 \%$ & $30 \%$ & $25 \%$ & $15 \%$ & 3 & 3 & 3.1 & 1.15 \\
\hline Treatment/Prevention & $5 \%$ & $5 \%$ & $15 \%$ & $15 \%$ & $60 \%$ & 5 & 5 & 4.2 & 1.21 \\
\hline Feasibility & $5 \%$ & $25 \%$ & $35 \%$ & $30 \%$ & $5 \%$ & 3 & 3 & 3.0 & 0.92 \\
\hline
\end{tabular}


Table 5.4 Result of the discrete choice experiment on criteria for prioritizing the HIV response

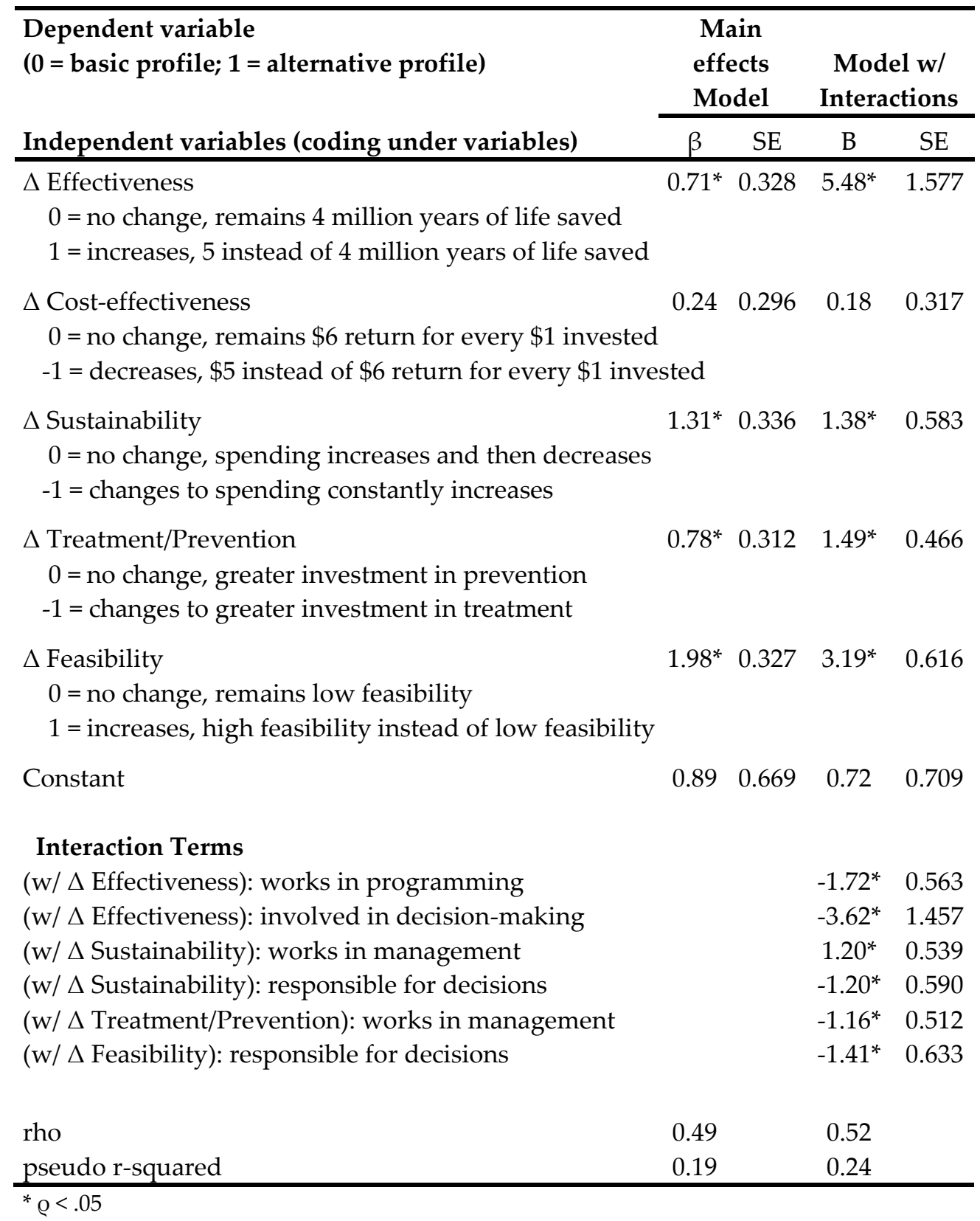




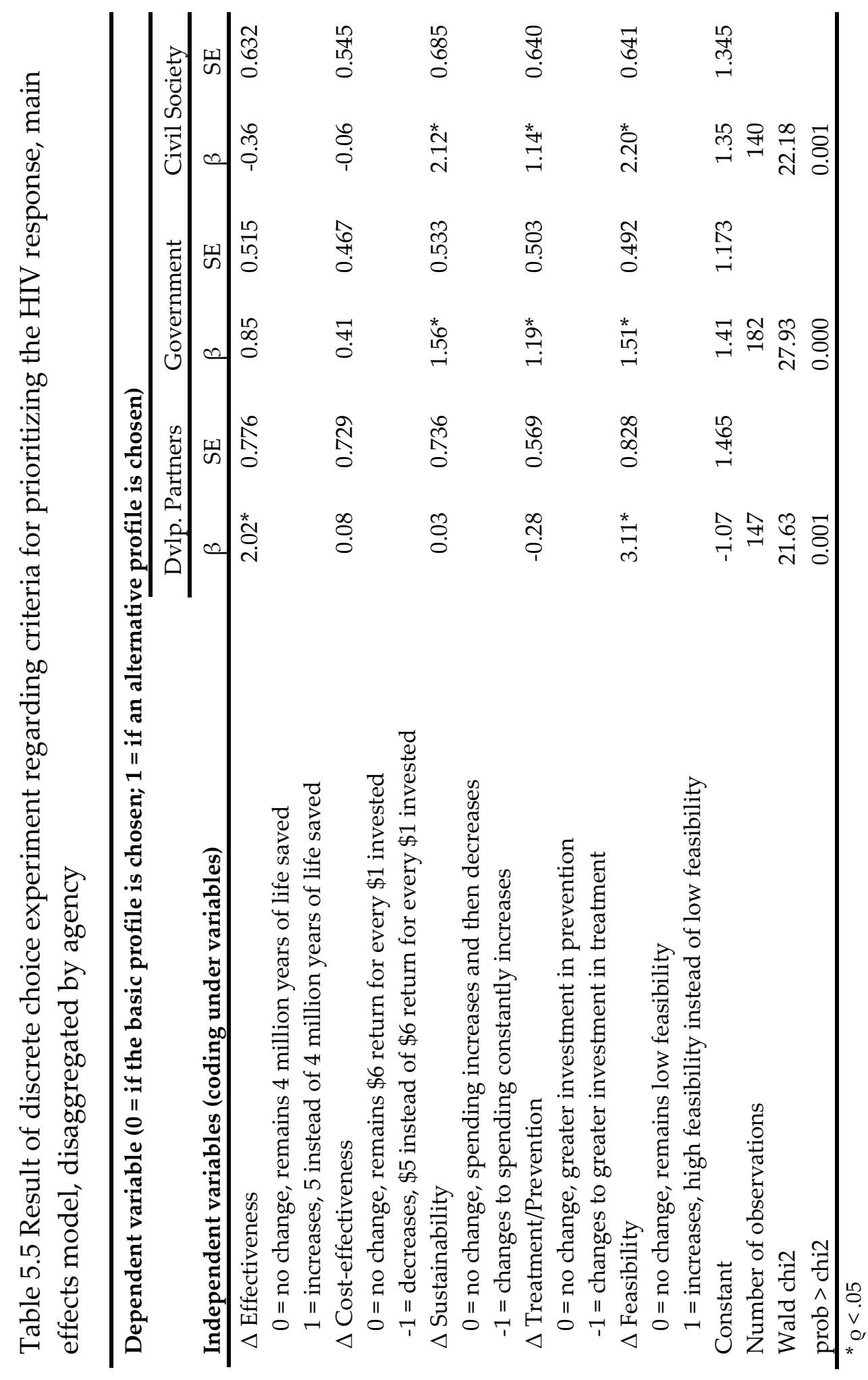


DCE analysis results by agency show that development partners, civil society, as well as government respondents all rank the feasibility criterion high. Sustainability, the ratio of treatment to prevention spending, and feasibility are in the top three criteria of both civil society and government respondents. Effectiveness is highly ranked by the development partners only. The coefficients of other criteria do not carry sufficient statistical weight to confidently say how they are ranked. Table 5.5 presents the ranking of criteria per each agency.

\subsection{Discussion}

All else being equal, participants prefer a program that is most feasible, frontloaded for sustainability, has a higher proportion of investment on prevention, saves more lives and prevents more infections, and is more cost-effective although this latter criterion does not show a statistically significant effect on the choices in the DCE.

The self-explicated ranking of criteria finds the "effectiveness" criterion to be the highest ranked criterion by respondents. This is in keeping with previous studies in rating importance of criteria in Asia that also found effectiveness to be the most important criterion for prioritizing interventions in the HIV response (Tromp, Prawiranegara, Subhan Riparev, et al., 2015; Youngkong et al., 2010). A systematic review of criteria in priority setting of HIV and health care also found effectiveness to be among the highest cited criteria in the literature (Guindo et al., 2012) (Chapter 2). Similar to these previous studies, during the self-explicated portion of this study, criteria were presented to participants as concepts without quantification, for example in terms of lives saved. However, during the DCE in this study, the effectiveness criterion dropped to fourth place according to its ranked importance 
to the respondents. This difference between the self-explicated ranking and DCE ranking may suggest the presence of social desirability bias in the self-explicated ranking. That is, when confronted with the general notion of a criterion such as effectiveness or sustainability, respondents may rely on their principles to determine its importance. However, when given more specific trade-off tasks during the DCE, for example to compare programs with nominal gains in lives saved at lower feasibility, the respondents may rely on their professional expertise to make their decisions. This phenomenon is further reinforced by the effectiveness criterion being consistently ranked highest by respondents from all different agency affiliations during self-explicated ranking, suggesting that the respondents' agency affiliations do not influence their decision. However, during the DCE only development partners ranked the effectiveness criterion high. Furthermore, DCE results show that the interaction of program effectiveness with professional characteristics of "working in programming" and "being involved in decisionmaking" significantly lower the ranking of the effectiveness criterion, suggesting that when the same respondents are asked to rank the program options, the ones with professional responsibilities in delivering program recommendations reconsider their priorities and lower their ranking of program effectiveness as a criterion vis-à-vis other criteria.

Another finding from the interaction results is the difference in ranking of criteria by respondents who have worked in management of HIV programs. Compared to the average response, those who worked in management rate prevention/treatment ratio lower and rate sustainability of programs higher. These results indicate some theoretical consideration in the decision-making of program managers based on financial models (Atun et al., 2016), giving a longer term view of the sustainability of the program even though it requires a large upfront investment. The program managers also consider a lower investment in prevention to offset a higher 
investment in treatment, which also indicates a theoretical approach given the mathematical models that suggest universal test and treatment programs could drive HIV eradication (Granich, Gilks, Dye, De Cock, \& Williams, 2017; Montaner et al., 2010) even though a more pragmatic view based on empirical evidence suggests many barriers in the cascade of care to link and retain patients in treatment (Gardner, McLees, Steiner, del Rio, \& Burman, 2011; Kilmarx \& MutasaApollo, 2012; Raymond, Hill, \& Pozniak, 2014).

The interaction terms also show that those responsible for decisions rate the feasibility and sustainability criteria lower than the average respondent. This is consistent with the traditional model of public service governance where decisionmakers are concerned with the outcomes of the programs they choose, and feasibility and sustainability are considerations for actors at different levels of their hierarchical organization (Hanson, 2012). Program planning is often sequenced from objective analysis, to activity planning, and ending with analysis of risks, with outcome results considered in the initial stages, and feasibility and sustainability considered in the later stages (Örtengren, 2004). This planning process is likely to have contributed to a program option that reflects more strongly the effectiveness criterion that is considered earlier in the process than other relevant criteria considered later in the planning process. Other iterative models of planning or greater involvement of stakeholders at all stages of planning may be needed to ensure relevant criteria are considered at appropriate decision points. This perspective is gaining traction in the recognition that the problems and solutions of public health cannot be solely owned by the government but require collaboration and engagement of multiple stakeholders (Institute of Medicine (US) Committee on Public Health Strategies to Improve Health, 2011). 
Feasibility and sustainability are ranked highly both in the DCE as well as the selfexplicated ranking. Although respondents from the civil society and government differ in the ordering of these two criteria in the DCE, their responses indicate that they agree the two criteria are the most important for prioritizing HIV programs. This result can be useful in the advocacy for greater involvement of the civil society in the priority setting process together with the government, since it brings to light that there are more points of agreement than differences between the two groups. While development partners agree with the government and civil society on the importance of the feasibility criterion, they consider the program effectiveness as their second most important criterion. Future priority setting processes may consider the level of importance of these criteria to different stakeholders, and develop program options that cater to their values. The transparency in options and weight of criteria according to different stakeholders will facilitate and focus discussions around trade-offs that need to be made and between whom.

The coefficient for the cost-effectiveness criterion was not significant in the regression analysis. In other words, the difference between the preference weight of the more cost-effective program and the less cost-effective program was not statistically significant. There could be two reasons for this: either we are unable to estimate the coefficients efficiently with the model used (e.g. too small difference between the levels of that criterion with no significant effect on the choice), or there is too much heterogeneity in the preferences for the cost-effectiveness criterion. The cost-effectiveness criterion however ranks low in self-explicated rankings, across all groups. This seems surprising if we consider the extensive use of the criterion for prioritization in healthcare, and guidelines developed for costeffectiveness analysis of healthcare programs (Edejer et al., 2003; Guindo et al., 2012; Neumann, 2004). However, a comparable phenomenon is observed in some Central and Eastern European countries with similar political-economy histories to 
Viet Nam, where cost-effectiveness is considered a "soft" criteria in healthcare priority setting (Kaló et al., 2013; Kaló, Gheorghe, Huic, Csanádi, \& Kristensen, 2016).

Between 2006 and 2010, the national HIV programs resulted in an estimated 401,600 fewer disease adjusted life years (DALY) at an estimated cost of $\$ 248$ for each DALY averted (Pham et al., 2015). The DCE results in this study indicate a preference for 5 million years of life saved from death and disease between 2016 and 2030, and \$315 for each DALY averted. These findings demonstrate that the stakeholders in this study prefer greater effectiveness of HIV programs in the future, but do not expect much change in the cost-effectiveness of the HIV programs. The 2014 national investment case (Viet Nam Ministry of Health, 2014) used two criteria of effectiveness and cost-effectiveness to rank several modeled HIV programs. Similar to findings of this study, effectiveness was prioritized over cost-effectives in the ranking of the choices. The investment case also considered a scenario where resource needs increase over time, as the "worst-case scenario", which is consistent with results of the DCE in this study on the sustainability criterion. The currently implemented national HIV program can also indicate the ratio of treatment to prevention spending. Recently prevention has accounted for close to $25 \%$ of funds of the HIV response, indicating that the national plan leans toward lower prevention spending, whereas findings of this study indicate a preference for greater prevention spending.

Overall, the DCE method is shown to be effective and feasible in establishing priorities in Viet Nam. It provides additional important information beyond what the self-explicated ranking of criteria provides, such as the comparative importance of one criterion against another. It also explains the direction of criteria that is preferred by the respondents. For example, whether they prefer greater prevention 
or greater treatment in the prevention/treatment ratio criterion. However, the DCE also requires a large number of respondents to make reliable estimates, which may not be feasible in countries with a small program and few people involved in decision-making at the central level to respond to the questionnaire. With few respondents, the number of criteria to be considered may be limited, jeopardizing the validity of the results.

This study has several strengths and limitations that need to be acknowledged. The strength of this study is in considering a broad set of criteria relevant to the country, and ranking them with experimental and self-explicated methods for improved accuracy and precision. Our study also involves multiple groups of stakeholders representing the different perspectives of those who should be involved in prioritizing Viet Nam's HIV response. This study has fewer participants in the DCE than other similar studies. Although we tried to reach a maximum number of actors with experience or expertise in decision-making on HIV programs, the HIV space in Viet Nam is ultimately limited by the size of the epidemic and response. Given the limited number of potential participants, and the desire to minimize the cognitive load of the DCE, a limited number of criteria are considered for prioritization from the full set.

This study occurs at a transition period in Viet Nam, as official development assistance to the HIV response is being reduced, and greater domestic investments including social health insurance are being mobilized to cover the gap left by the donors. The stakeholders' ranking of the criteria for prioritizing HIV programs presented here may be a reflection of the current context in Viet Nam, which could change in the future. 


\subsection{Conclusion}

Findings of this study show that there are greater similarities between the ranking of criteria by government and civil society than there are differences. The process and results in elicitation of the importance of the criteria can inform future policy dialogues between the stakeholders to find common grounds in priority setting. The results also highlight the need to reconsider the classical hierarchical models of planning in Viet Nam, and utilize innovative models of planning that allow inputs of informed stakeholders at relevant stages of the HIV program planning process. The results may also be useful for other developing countries in a transition period to visit or revisit the criteria used to prioritize their HIV programs. In donor supported countries, the transparent process of eliciting criteria for HIV program prioritization can be an additional requirement for funding proposals that demonstrates wide stakeholder consultation, and evidence-based planning and prioritization.

As Viet Nam moves closer to becoming an upper-middle-income country, and donors transition away from direct support of the HIV response, the importance of certain criteria for prioritizing the HIV program package will need to be reevaluated. Cost-effectiveness is one criterion used prominently in the past investment case analysis of Viet Nam's HIV response, but ranks lower in this study. Going forward, this criterion should again be considered centrally once programs transition from donor support to domestic financing, and standalone HIV programs integrate back into the general healthcare system, and evidence becomes available on the cost and effectiveness of this newly integrated program structure. 


\section{Chapter 6: General Discussion}




\subsection{Introduction}

The principle focus of this dissertation is on the appropriateness and applicability of the MCDA framework to prioritizing the HIV response. It takes this perspective, while considering the two problems that afflict prioritization processes: lack of stakeholder involvement and lack of quality evidence for decision-making. In the introduction chapter of this dissertation four questions were formulated for investigation. These four questions are related to the four objectives for this dissertation: 1) identify the globally relevant criteria to prioritize the programs, policies, investments, workforce and technologies that are utilized in responding to the HIV epidemic, 2) assess the extent to which reliable and good quality evidence is available, accessible and used in planning for the HIV response, 3) understand the difference that the inclusion of diverse stakeholders make in decision-making processes of the HIV response, 4) measure the observed importance that different stakeholders attach to relevant criteria for prioritizing the HIV response.

To meet the objectives of the dissertation, four studies have been conducted. Thus, the four objectives are addressed in the four successive chapters of the dissertation respectively. Chapter 2 of this dissertation systematically reviews the criteria relevant to the HIV response. Chapter 3 reviews the quality and extent of evidence used in planning the HIV response. Chapter 4 explores the underlying causes of gaps in evidence, and the value provided by involvement of stakeholders in the process of priority setting. Chapter 5 conducts an experiment to study the preferences and trade-offs made between different HIV programs by multiple stakeholders and decision-makers. 


\subsection{Main statements based on research findings}

This section presents the key crosscutting findings from the various chapters of this dissertation. These findings are presented in the form of five statements followed by a short elaboration of the statement's origins, its policy implications, and any suggestions for future research.

\section{Statement 1: To be effective, the process of priority setting of the HIV response requires an iterative, multi-stakeholder approach}

Findings of this dissertation demonstrate that in the numerous planning stages of an HIV program, it is important to consider who is involved, because who is involved is ultimately related to what criteria are considered, and the criteria considered determine the program components that are prioritized (Chapter 5). Strategic involvement of multiple stakeholders in various stages of the planning process can ensure that relevant criteria are considered comprehensively, accurately defined and rated in their importance. Involvement of multiple stakeholders however also introduces conflicts in definition and rating of criteria for priority setting (Chapter 4). These conflicts need to be addressed not by exclusion of stakeholders whose definitions or rating of criteria differ from the norm, or coercion to force a common viewpoint, but a systematic way of eliciting and weighting criteria from multiple perspectives (Chapter 4). The DCE in this dissertation demonstrated an effective method to bring clarity to differences in the ranking of criteria. DCE can be used to either weight criteria for consideration of different voices, or to show that there may be similarity among stakeholders, and in either case their inclusion would further legitimize the process of priority setting. DCE is also preferred over self-explicated ranking methods of weighting criteria, as the latter may suffer from social desirability bias (Chapter 5). 
The linkage between health outcome effects and the decision-making process are not explored in this dissertation. However, other research in corporate behavioral strategy demonstrates that investments in decision-making processes have greater returns than investments on evidence generation and analytics (Lovallo \& Sibony, 2010). That is not to say that evidence generation and analytical work is unimportant. Rather, it says that there is a strong relationship between process and analysis, and "superb analysis is useless unless the decision process gives it a fair hearing" (Lovallo \& Sibony, 2010). Recommendations based on findings in Chapter 4 of this dissertation underline the direct and indirect value of the process of representing the decision analysis jointly. Another process recommendation, based on findings on the importance of criteria at various levels of planning, is to consider an iterative model of planning to ensure that relevant criteria are considered at appropriate decision points (Chapter 5). Steele et al observe that in many real examples of decision-making, criteria are not easily understood by stakeholders (Steele, Carmel, Cross, \& Wilcox, 2009). Therefore the iteration process also applies (and was applied in Chapter 4 and 5) to ensuring a common and complete understanding of the choices and criteria by the stakeholders. This iterative approach to decision process is consistent with recommendations of operational research in the application of MCDA in spatial planning processes (Monnikhof \& Bots, 2000). These recommendations taken together reinforce the applicability of the MCDA framework, which includes procedures to identify courses of actions in a manner that is analytically robust and consistent in light of the available evidence and stakeholder preferences (Marttunen, 2011).

An important recommendation based on the review of evidence in past plans is for the generation of program evaluation evidence to be synchronized with the planning process so that timely evidence is used effectively in priority setting 
(Chapter 3). Overall, the findings of this dissertation show that an effective approach to priority setting of the HIV response requires a review of criteria, modeling of program options based on quality evidence, and analysis of criteria weights for prioritizing program options (Chapter 2, 4, 5).

This dissertation has studied the priority setting processes of the HIV response at a moment in history when AIDS is in transition from a global emergency to a chronic disease (World Health Organization, 2017). The evidence reviewed in the dissertation has spanned globally, although specific chapters have concentrated on case studies to explore interpretation and use of criteria by multiple stakeholders in a priority setting process while isolating variance in social, political and economic factors. While the guidance and instruments developed in the course of this dissertation have demonstrated utility and reliability, their relevance to other phases of the HIV epidemic, to different social, political and economic settings, and to other emerging or ongoing epidemics will require further investigation.

\section{Statement 2: The global fall in HIV funding and the reintegration of HIV programs into the health sector have renewed the focus on feasible and sustainable programs}

The systematic review of criteria conducted in Chapter 2 of this dissertation suggested that there is a noticeable difference in the elicitation of the criteria of feasibility and sustainability before and after 2007. Chapter 2 hypothesized that the less frequent elicitation of these criteria prior to 2007 may be due to a gradual movement away from reason- or value-based decision-making. The reason-based decision-making occurs when for example politicians are pressured to seek and construct a 'reason' to justify their choice in the context of deficient evidence on 
criteria for evaluating their options (Goodwin \& Wright, 2004; Keren \& Bruin, 2003; Shafir, Simonson, \& Tversky, 1993). The hypothesis then goes on to predict that evidence-based decision-making and programming is aided by improved monitoring and evaluation systems that produce evidence along the relevant criteria. An alternative hypothesis was also proposed in Chapter 2 that the increasing availability of pharmacological HIV treatment and prevention methods, with more reliable outcomes and proven feasibility in various settings, might have contributed to a decline in the emphasis on feasibility criteria. In Chapter 3, we saw that the first of these two hypotheses is more probable with overall evidence use increasing in HIV planning, while evidence on sustainability and operations research to assess feasibility remains weak.

More recently, the importance of feasibility has come to the forefront of the discussion on the selection of health interventions and activities in LMIC (Diaconu et al., 2017; Guindo et al., 2012; Hosek et al., 2013; Niëns, 2014; Terwindt, Rajan, \& Soucat, 2016). In Chapter 4 of this dissertation, the majority of key informants, including all at-risk community members interviewed, mentioned the feasibility criterion. And when a group of decision-makers and stakeholders in Vietnam were asked to rank the criteria most important for focusing HIV programs, feasibility and sustainability came out high on the list (Chapter 5). In that experiment, operational definitions were attached to the criteria and linked to program options with measurable characteristics. The high ranking of feasibility and sustainability in the experiment further reinforces the notion that criteria that are well defined and measured are ones that will get used in this era of evidence-based planning. The implication here is that if there are no measures for a program, an intervention, or an at-risk population, then they may be neglected. Or as the UN Secretary General Ban Ki Moon puts it, "if you're not counted, you don't count" (Moon, 2016). 
The systematic review of literature prior to 2014 in Chapter 2 of this dissertation showed that lower income countries elicit feasibility less frequently than upper income countries, and sustainability is nearly equally elicited in both income groups. The feasibility and sustainability are far more frequently elicited in normative guidance than in literature describing current programs, attributed to the lack of consideration of resource needs in the HIV response. The recent observation of frequent elicitation of these criteria (Chapter 4) and their high ranking in selecting HIV programs (Chapter 5) in Viet Nam coincides with the decline in donor spending to address HIV in LMIC since 2014 (Chapter 1). The transition and integration of donor funded programs into domestic funded health sector are likely refocusing attention on resource allocation in the HIV response, and contributing to the resurgence in consideration of feasibility and sustainability of HIV programs.

The resurgence of feasibility and sustainability is reassuring in underlining a pragmatic approach with a long-term view of the HIV response to end AIDS as a public health threat. There are two consequences to this renewed focus to be watched with caution as countries implement programs with criteria of feasibility and sustainability foremost in their sight.

First, as noted in other statements, who defines what feasibility means and how it is defined matters. It needs to be underlined again here that involvement of stakeholders in different stages of priority setting will be critical for a fair and rational outcomes. Oversight at the highest level of government will be required to ensure that the progress toward feasible programs does not translate to a decentralization of decision-making to program experts who may exclude lay stakeholders due to the lack of technical expertise, but is rather an upward 
channeling of information for multi-stakeholder policy setting (Rayner, 2003). This former technocratic approach to decision-making is a means of exclusion of nonstate actors and stakeholders without technical expertise from the priority setting process (Boivin, Lehoux, Burgers, \& Grol, 2014; Kantrowitz, 1975).

Second, the higher ranking of feasibility and sustainability in terms of importance for prioritizing HIV programs, comes with an intertemporal trade-off with other criteria such as cost-effectiveness, epidemic effect, or equity being ranked lower in importance (Bansal, Chapardar, \& Gehman, 2016). This is an expected and accepted outcome of fair decision-making based on multi-stakeholder decided criteria (Danziger, Montal, \& Barkan, 2012). However, it should also be clear that this increased emphasis on pragmatism would bear a cost, financially and also in terms of potential years of life saved from disease (Marsh et al., 2016). In this situation, a transparent process based on evidence along the clearly defined criteria, will support HIV policies as trade-offs are made. It will also ensure accountability to the stakeholders who are ultimately affected by the implemented programs (Yamin, 2008).

The systematic review of criteria in Chapter 2 included those from both lower and middle-income countries as well as upper income countries. The observations from the LMIC were further investigated with a review of plans in lower and middleincome countries in Sub-Saharan Africa (Chapter 3). Chapter 4 and 5 focused on one LMIC, Viet Nam, to further validate and update the trend toward refocusing the HIV response on feasible and sustainable programs. The criteria emerging from the systematic review in Chapter 2 were ranked based on the frequency of their appearance in literature. The criteria were ranked in Chapter 5 based on observed choices of decision-makers in an experimental setting. This difference in method, and the geographical coverage in the application of the methods, prevents us from 
comparing the two rankings of criteria in Chapters 2 and 5. While the case of Viet Nam's HIV response may not be generalized to all LMIC with an HIV epidemic, the trends observed in Viet Nam as well as reports and review from other LMIC warrant further investigation of the renewed focus on feasible and sustainable programs in other countries.

\section{Statement 3: The use of evidence in HIV planning and policy- making has been increasing over the years, particularly use of data on drivers of the epidemic}

Chapter 2 of this dissertation proposed that weak health information systems and stigma for key populations at risk of HIV present some barriers to effective application of the criteria in resource poor settings. It does so by limiting the available evidence base for operationalizing criteria, which may deter decisionmakers in considering criteria without adequate measures. This condition was tested in Chapter 3 where a review of national strategic plans for HIV and AIDS control and prevention in Sub-Saharan Africa revealed that with the increasing strength of monitoring and evaluation systems over the years, the type of evidence used today in planning is more diverse and of higher quality than in the past. Therefore, the availability of evidence as a precondition for application of criteria is met.

According to the findings of Chapter 3 in this dissertation, while evidence use is increasing, there is still considerable room for improvement in breadth and quality of evidence used in national HIV plans. In particular, there is poor quality and coverage of evidence for key populations at risk of HIV, which is attributed to inadequate surveillance methods for these groups (Chapter 4). Although 
monitoring and evaluation systems have increased in strength over the years (Peersman et al., 2009), findings in Chapter 3 demonstrated that there is more evidence that is used in planning which comes from HIV program monitoring than from HIV program evaluation and research. Impact evaluations and operations research were two particular types of evidence that were sought in the review of national HIV plans (Chapter 3), but were noticeably absent. Evidence on operation and impact of HIV programs tells planners if the programs are implemented the right way, and if they are having an effect in curbing the HIV epidemic (UNAIDS, 2008). Therefore, it is critical for good planning to have evidence available from operations research and impact evaluations (Hargreaves et al., 2016; Malhotra \& Zodpey, 2010).

Analysis of choices of program managers in selecting hypothetical HIV program options in Chapter 5 indicated that they may be relying on modeled data instead of operations research to decide on their preferred program options, based on criteria of sustainability and increased investments in treatment over prevention. This means that the use of modeled data is preferred by managers over operations research, and clarifies the lack of operations research in national strategic plans (Chapter 3).

As noted in the introduction of this dissertation, donor funding for the HIV response has been declining in recent years (KFF \& UNAIDS, 2016; UNAIDS, 2016d). With reduced investments from bilateral and multilateral sources, the need for the involvement of these donors in the national HIV program priority setting processes would also decrease. And with fewer donors at the national decisionmaking table, the autonomy of national stakeholders to negotiate the package of interventions that make up the national HIV response become stronger, and less pressured by supranational level interests (Henriksson et al., 2017; OECD, 2011). 
This less politicized negotiation process, together with improved availability of evidence, may potentially contribute to the observed increase in reliance on evidence to drive the HIV planning and policy making (Dean \& Sharfman, 1996). Another contributing factor to the use of evidence in HIV planning and policy may be a gradual turnover of leadership, between public health leaders who started in the HIV response reapplying their knowhow from other health challenges, to a new generation of leaders who have worked their way up from HIV programming to policy making and are acutely aware of what programs work, and the evidence needs to scale those programs nationally (Pfeffer \& Sutton, 2006).

Notwithstanding the gaps in quality and coverage of evidence used in planning of HIV programs, the overall trend in increasing use of evidence is encouraging, but it also implies an imminent challenge for decision-makers to process the volume of information and exercise sound judgment (Christodoulou, Karacapilidis, Tzagarakis, Dimitrova, \& de la Calle, 2014). Considering the multiple criteria that exist in evaluating HIV programs, and the high stakes of the decisions, a strategy for effective management and use of the information for decision-making is necessary. The strategy will need to consider the multiple stakeholders who should be involved in the decision-making and the costs of transfer of- and their orientation with- the available evidence. Another challenge will be the analysis of the diversity of information, including both qualitative and quantitative data. The strategy may opt to use innovative analytical methods or mathematical modeling of scenarios to aid decision-makers with processing of the volume and diversity of data.

Evidence availability and use in planning is only one component in ensuring desirable outcomes. Further evaluation is needed to see if evidence-based planning produces better outcomes than ad-hoc planning (Chapter 3). In addition to volume 
and variety of evidence becoming available for use, the speed with which evidence is generated also requires further investigation.

\section{Statement 4: The gaps and variance in quality evidence on key at- risk groups take the focus away from these populations in national strategic plans and programs}

In Chapter 2 of this dissertation a systematic review of literature to identify criteria used in the HIV response priority setting revealed low attention to high-risk populations. High-risk populations ranked in the lower half of all criteria elicited, irrespective of stratification, and even in concentrated epidemic countries, where by definition the epidemic is concentrated among high-risk populations. This phenomenon prompted further investigation in Chapter 4, of the extent to which key populations are considered in national strategic plans for HIV control and prevention, and what evidence on key populations is available and used. A subsequent review of national strategic plans in Chapter 3 noted a gap in evidence on key populations at risk of HIV, particularly men who have sex with men. Drivers of this gap in evidence were explored in Chapter 4 through interviews with surveillance specialists and members of the at-risk community. These discussions revealed stigmatization of subjects as a main deterrent to participation in surveillance activities, which in turn contributes to reduced validity, use, and efficiency in evidence generated from surveillance. Additionally, the identified conflicting views on criteria between key populations and technical experts on selection of methods of surveillance, and the key populations' lack of technical skills in surveillance methods, may be contributing to these stakeholders not being able or allowed to participate in evidence generation about their own community. 
Without the generation of evidence by the community and for the community, resulting data may not be trusted for use in the national planning process (Innes \& Booher, 2004). And without evidence, the national plans will continue the cycle of taking attention away from the key populations. Psychologist Daniel Kahneman has labeled this latter concept as follows: "what you see is all there is" (Kahneman, 2011). It refers to the human bias in using the readily available information and ignoring the quality and coverage of evidence needed to make informed rational decisions.

A critical consequence of the reduced focus on key populations in national plans and programs is attenuated progress in controlling HIV in the concentrated epidemics (Oberth \& Whiteside, 2016). Leaving epidemics among key at-risk populations unchecked can result in a resurgence of HIV cases in the community, but also create the potential spillover effect of the epidemic to the lower risk vulnerable groups, such as intimate partners of the key at-risk populations, as well as infants born to the women living with HIV (UNDP, 2015). Programs to curb infections among key at-risk populations in generalized epidemics can also benefit the overall epidemic acting through modes of transmission.

Recent normative guidance to close the evidence gap and support improved decision-making by seeking to engage with patients and consumers is encouraging (Califf et al., 2016). Implementation research in lay-person engagement in HIV surveillance and evidence generation on key at-risk populations is also promising (Safarnejad et al., 2017). Further piloting and scaling up of innovative methods to engage the key at-risk populations in surveillance of communicable diseases is recommended to address the persistent gaps in evidence. While engagement of the community in community monitoring also addresses the stigma and discrimination barriers, a more direct approach to stigma reduction is 
recommended in addition to create an enabling environment for prevention and harm reduction programs to work. A promising method that has been demonstrated to durably reduce stigma for transgender people in the United States is door-to-door canvassing (Broockman \& Kalla, 2016).

The review of national plans in Chapter 3 of this dissertation, where the gaps in evidence on key at-risk populations were noted, and the subsequent exploration of the underlying reasons for the gaps in Chapter 4, focused on mostly LMIC and where the majority of people living with HIV reside. Therefore, caution should be exercised in generalizing the statement to all countries. Further research in uppermiddle- and upper- income countries would be required to explore if gaps in evidence on key at-risk groups exist there. Specific case studies could corroborate if the explanatory factors for at-risk group evidence gaps identified in this dissertation translate well to other countries.

\section{Statement 5: Epidemic control and reduction of stigma for key at- risk groups requires their involvement in the HIV response priority setting process}

In Chapter 2 of this dissertation, social justice emerged as a distinct theme in the review of criteria for priority setting in the HIV response, with stigma and discrimination as both a cause and a consequence of the epidemic. Stigma and discrimination was in fact frequently elicited as a criterion in Chapter 2, particularly in LMIC. This frequent elicitation is related to low knowledge among the population about modes of transmission of $\mathrm{HIV}$, and consequent negative attitudes toward people living with or at high risk of HIV. The evidence available on stigma and discrimination, programs on knowledge transfer to the community, 
and involvement of key at-risk populations in planning were further explored in Chapter 3 and 4 .

The review of national strategic plans in Chapter 3 revealed that while evidence use is increasing, evidence on key populations is not adequate, and there is low involvement of key populations in the planning process to affect improved evidence use. So what are the incentives or disincentives of involvement of key populations in the priority setting process? In Chapter 4, we observed that the involvement of key populations ensures that a broad and comprehensive set of criteria are considered. Participants from the key population group who were interviewed for Chapter 4 considered that 'community participation' in surveillance is an important criterion for selection of surveillance methods. However, no technical experts or health managers mentioned 'community participation' as a criterion. This further reinforces the notion that key populations are not included in technical dialogues on evidence generation, because the experts and managers do not consider it important (Chapter 4). It also reflects a broader trend in participation of citizenry from different perspectives in decision-making. In his thesis, Toward a Rational Society, Habermas posits that since World War II science and rationalization has been becoming mainstreamed and formalized in political discussions, so it further excludes the citizens from purely political discussions because politicians are orienting themselves to strictly scientific arguments in the exercise of their public function (Habermas, 1971).

By delegating political decisions to the technical context, important value decisions are taken out of the hands of the citizen stakeholders. With further decentralization and delegation of decision-making, the space for decision criteria of relevant stakeholders becomes successively smaller, and the number of the citizen participants able to participate in the process becomes successively smaller. This 
kind of technocratic decision-making, where the experts decide, is often applied to scientific decisions. Sociologist Dorothy Nelkin questions the ability of technocratic approach to settle value conflicts, concluding that "increased technical information often tends to increase polarization" in already conflict-ridden fundamental political choices (Nelkin, 1981).

The forewarning of marginalization of key stakeholders in technocratic decisionmaking may imply a solution that involves centralized political decision-making at a level that allows access to the widest stakeholder involvement and input. However, studies point out that a greater number of citizen stakeholders who can be involved, is related to lower level of their active involvement (Cogan, Sharpe, \& Hertzberg, 1986) and greater deliberation may even lead to further entrenchment of previously held views (Abelson, Eyles, et al., 2003). Thus, while decentralized decision-making with formalized technical roles for participating stakeholders or centralized decision-making with passive consultation with stakeholders may be desirable from the perspective of reaching consensus, or at best to show accountability, from the perspective of a fair and legitimate process, they are not. Given the previously mentioned importance of community participation in surveillance activities, an alternative recommended policy is for investments to be made in technical literacy of community-based organizations to strengthen their role in decision-making or grass roots movements for community-driven policy decisions to be made in research, science and technology that contribute to epidemic control. 


\subsection{Concluding remarks}

This dissertation has looked at the issue of involvement of stakeholders in the decision-making process of the HIV response in terms feasibility of such involvement, gains in improved evidence, and the acceptability of the outcomes of the decisions made. It has not looked at the costs associated with greater involvement of a diversity of stakeholders in the priority setting process. It has also not assessed the existing capacity, readiness, or willingness of stakeholders to participate in decisions. These assumptions vary from country to country, and between key populations groups and they should be examined carefully prior to deciding on a course of action in stakeholder involvement.

Overall, this dissertation has shown that the elements of the MCDA framework are applicable to prioritizing the HIV response. There is readiness for the application of the framework in terms of evidence availability, defined criteria, and feasibility of involvement of multiple stakeholders in the process, with noted gaps and areas of improvement. As the financial resources and political will to end the AIDS epidemic as a public health threat wanes, findings and recommendations of this dissertation can give guidance on a fair, accountable and transparent path to prioritizing the HIV response to treat the most vulnerable and to leave no one behind. 


\section{References}

Abdul-Quader, A. S., Baughman, A. L., \& Hladik, W. (2014). Estimating the size of key populations. Current Opinion in HIV and AIDS, 9(2), 107-114. doi:10.1097/COH.0000000000000041

Abelson, J., Eyles, J., McLeod, C. B., Collins, P., McMullan, C., \& Forest, P.-G. (2003). Does deliberation make a difference? Results from a citizens panel study of health goals priority setting. Health Policy, 66(1), 95-106. doi:https://doi.org/10.1016/S0168-8510(03)00048-4

Abelson, J., Forest, P.-G., Eyles, J., Smith, P., Martin, E., \& Gauvin, F.-P. (2003). Deliberations about deliberative methods: issues in the design and evaluation of public participation processes. Social Science $\mathcal{E}$ Medicine, 57(2), 239-251. doi:https://doi.org/10.1016/S0277-9536(02)00343-X

Adrien, M.-H., Bamberger, M., Conner, R. F., Djokovic-Papic, D., Hancioglu, A., Jankovic, V., ... Vukovic, A. (2008). Bridging the gap: The role of monitoring and evaluation in evidence-based policy making (p. 219). Geneva. Retrieved from https://www.unicef.org/ceecis/evidence_based_policy_making.pdf

Ainsworth, M., \& Teokul, W. (2000). Breaking the silence: setting realistic priorities for AIDS control in less-developed countries. Lancet, 356(9223), 55-60. doi:10.1016/S0140-6736(00)02440-5

Alfven, T., McDougal, L., Frescura, L., Aran, C., Amler, P., \& Gill, W. (2014). A decade of investments in monitoring the HIV epidemic: how far have we come? A descriptive analysis. Health Research Policy and Systems, 12(1), 62. doi:10.1186/1478-4505-12-62

Alistar, S. S., \& Brandeau, M. L. (2012). Decision making for HIV prevention and treatment scale up: bridging the gap between theory and practice. Medical Decision Making : An International Journal of the Society for Medical Decision Making, 32(1), 105-17. doi:10.1177/0272989X10391808

Alistar, S. S., Brandeau, M. L., \& Beck, E. J. (2013). REACH: A Practical HIV Resource Allocation Tool for Decision Makers. In G. S. Zaric (Ed.), Operations Research and Health Care Policy (pp. 201-223). New York, NY: Springer New York. doi:10.1007/978-1-4614-6507-2_10 
Andrews, J. (2013). Prioritization Criteria Methodology for Future Research Needs Proposals Within the Effective Health Care Program: PiCMe-Prioritization Criteria Methods. Methods Future Research Needs Reports, No. 10. Rockville, MD: Agency for Healthcare Research and Quality (AHRQ). Retrieved from https://www.ncbi.nlm.nih.gov/pubmed/23367528

Atun, R., Chang, A. Y., Ogbuoji, O., Silva, S., Resch, S., Hontelez, J., \& Bärnighausen, T. (2016). Long-term financing needs for HIV control in subSaharan Africa in 2015-2050: a modelling study. BMJ Open, 6(3), e009656. doi:10.1136/bmjopen-2015-009656

Baltussen, R., Mikkelsen, E., Tromp, N., Hurtig, A., Byskov, J., Olsen, O., ... Norheim, O. F. (2013). Balancing efficiency, equity and feasibility of HIV treatment in South Africa - development of programmatic guidance. Cost Effectiveness and Resource Allocation: C/E, 11(1), 26. doi:10.1186/1478-7547-1126

Baltussen, R., \& Niessen, L. (2006). Priority setting of health interventions: the need for multi-criteria decision analysis. Cost Effectiveness and Resource Allocation, 4, 14. doi:10.1186/1478-7547-4-14

Baltussen, R., Stolk, E., Chisholm, D., \& Aikins, M. (2006). Towards a multi-criteria approach for priority setting: an application to Ghana. Health Economics, 15(7), 689-96. doi:10.1002/hec.1092

Baltussen, R., Youngkong, S., Paolucci, F., \& Niessen, L. (2010). Multi-criteria decision analysis to prioritize health interventions: Capitalizing on first experiences. Health Policy, 96(3), 262-264. doi:10.1016/j.healthpol.2010.01.009

Bansal, T., Chapardar, H., \& Gehman, J. (2016). Tradeoffs in SustainabilityOriented Innovations. Retrieved May 15, 2017, from http://sloanreview.mit.edu/article/tradeoffs-in-sustainability-orientedinnovations/

Barre-Sinoussi, F., Chermann, J. C., Rey, F., Nugeyre, M. T., Chamaret, S., Gruest, J., ... Montagnier, L. (1983). Isolation of a T-lymphotropic retrovirus from a patient at risk for acquired immune deficiency syndrome (AIDS). Science (New York, N.Y.), 220(4599), 868-871. 
Beeker, C., Guenther-Grey, C., \& Raj, A. (1998). Community empowerment paradigm drift and the primary prevention of HIV/AIDS. Social Science $\mathcal{E}$ Medicine, 46(7), 831-842. doi:10.1016/S0277-9536(97)00208-6

Benatar, S. (2006). Facing ethical challenges in rolling out antiretroviral treatment in resource-poor countries: comment on "They call it "patient selection" in Khayelitsha. Cambridge Quarterly of Healthcare Ethics : CQ : The International Journal of Healthcare Ethics Committees, 15(3), 322-330.

Bennett, S., Boerma, J. T., \& Brugha, R. (2006). Scaling up HIV/AIDS evaluation. The Lancet, 367(9504), 79-82. doi:10.1016/S0140-6736(06)67928-2

Bennett, S., \& Chanfreau, C. (2005). Approaches to rationing antiretroviral treatment: ethical and equity implications. Bulletin of the World Health Organization, 83(7), 541-547.

Boerma, J. T., \& Stansfield, S. K. (2007). Health statistics now: are we making the right investments? The Lancet, 369(9563), 779-786. doi:10.1016/S01406736(07)60364-X

Boivin, A., Lehoux, P., Burgers, J., \& Grol, R. (2014). What Are the Key Ingredients for Effective Public Involvement in Health Care Improvement and Policy Decisions? A Randomized Trial Process Evaluation. The Milbank Quarterly, 92(2), 319-350. Retrieved from http://www.jstor.org/stable/24369970

Boța-Avram, C. (2013). The Interplay Between Governance and Global Competitiveness: Evidence from a Cross-Country Survey. Transition Studies Review, 20(3), 381-388. doi:10.1007/s11300-013-0291-5

Bradshaw, D., Chopra, M., Kerber, K., Lawn, J. E., Bamford, L., Moodley, J., ... Velaphi, S. (2008). Every death counts: use of mortality audit data for decision making to save the lives of mothers, babies, and children in South Africa. Lancet, 371(9620), 1294-1304. doi:10.1016/S0140-6736(08)60564-4

Briones-Vozmediano, E., Vives-Cases, C., \& Peiro-Perez, R. (2012). Gender sensitivity in national health plans in Latin America and the European Union. Health Policy, 106(1), 88-96. doi:10.1016/j.healthpol.2012.03.001

Broockman, D., \& Kalla, J. (2016). Durably reducing transphobia: A field experiment on door-to-door canvassing. Science, 352(6282), 220-224. doi:10.1126/science.aad 9713 
Brownson, R. C., Chriqui, J. F., \& Stamatakis, K. A. (2009). Understanding Evidence-Based Public Health Policy. American Journal of Public Health, 99(9), 1576-1583. doi:10.2105/AJPH.2008.156224

Bryson, M., Duclos, P., Jolly, A., \& Bryson, J. (2010). A systematic review of national immunization policy making processes. Vaccine, 28(Suppl 1), A6-12. doi:10.1016/j.vaccine.2010.02.026

Burchett, H. E. D., Mounier-Jack, S., Griffiths, U. K., \& Mills, A. J. (2012). National decision-making on adopting new vaccines: a systematic review. Health Policy and Planning, 27(suppl 2), ii62-ii76. doi:10.1093/heapol/czr049

Burgess, R. G. (1984). In the Field: An Introduction to Field Research. London, England: Allen \& Unwin.

Cabassi, J. (2004). Code of Good Practice for NGOs Responding to HIVIAIDS. (D. Wilson, Ed.). Geneva, Switzerland: Oxfam Publishing.

Califf, R. M., Robb, M. A., Bindman, A. B., Briggs, J. P., Collins, F. S., Conway, P. H., .. Sherman, R. E. (2016). Transforming Evidence Generation to Support Health and Health Care Decisions. New England Journal of Medicine, 375(24), 2395-2400. doi:10.1056/NEJMsb1610128

Carey, T. S., Sanders, G. D., Viswanathan, M., Trikalinos, T. A., Kato, E., \& Chang, S. (2012). Framework for Considering Study Designs for Future Research Needs. Methods Future Research Needs Reports, No. 8. Rockville, MD: Agency for Healthcare Research and Quality (AHRQ).

Carpiano, R. M. (2009). Come take a walk with me: The "Go-Along" interview as a novel method for studying the implications of place for health and wellbeing. Health \& Place, 15(1), 263-272.

doi:http://dx.doi.org/10.1016/j.healthplace.2008.05.003

Catania, J. A., Canchola, J., \& Pollack, L. (2002). Using survey data to estimate the population size and distribution of MSM. Retrieved June 24, 2015, from https:/www.cdph.ca.gov/programs/aids/Documents/RPT2002UsingSurveyD atatoEstPopMSM.pdf

Center for Disease Control and Prevention. (2010). Distinguishing Public Health Research and Public Health Nonresearch (p. 13). Atlanta, GA. Retrieved from 
https://www.cdc.gov/od/science/integrity/docs/cdc-policy-distinguishingpublic-health-research-nonresearch.pdf

Centers for Disease Control. (1982). Update on acquired immune deficiency syndrome (AIDS)--United States. MMWR. Morbidity and Mortality Weekly Report, 31(37), 507-508.

Christodoulou, S., Karacapilidis, N., Tzagarakis, M., Dimitrova, V., \& de la Calle, G. (2014). Data Intensiveness and Cognitive Complexity in Contemporary Collaboration and Decision Making Settings. In N. Karacapilidis (Ed.), Mastering Data-Intensive Collaboration and Decision Making: Research and practical applications in the Dicode project (pp. 17-48). Springer International Publishing. Retrieved from https://books.google.com.vn/books?id=ZI64BAAAQBAJ

Ciliska, D., Thomas, H., \& Buffett, C. (2012). An Introduction to Evidence-Informed Public Health and A Compendium of Critical Appraisal Tools for Public Health Practice (Revised). Hamilton, ON: National Collaborating Centre for Methods and Tools. Retrieved from http://www.nccmt.ca/pubs/IntroEIPH_compendiumENG_web.pdf

Cleary, S. (2010). Equity and efficiency in scaling up access to HIV-related interventions in resource-limited settings. Current Opinion in HIV and AIDS, 5(3), 210-214. doi:10.1097/COH.0b013e3283384a6f

Cogan, A., Sharpe, S., \& Hertzberg, J. (1986). Citizen Participation. In F. S. So, I. Hand, \& B. D. McDowell (Eds.), The Practice of Regional and State Planning (pp. 283-308). Chicago, Ill: American Planning Association.

Country Coordinating Mechanism Vietnam. (2015). CCM MEMBER LIST. Retrieved March 28, 2017, from http://ccmvietnam.gov.vn/index.php?option=com_content\&view=category\&l ayout $=$ blog\&id $=65 \&$ Itemid $=157 \&$ lang $=$ en

Creswell, J. W. (2009). Research Design: Qualitative, Quantitative, and Mixed Methods Approaches (3rd ed., p. 260). SAGE Publications. Retrieved from https://books.google.com.vn/books?id=bttwENORfhgC

Cronbach, L. J. (1951). Coefficient alpha and the internal structure of tests. Psychometrika, 16(3), 297-334. doi:10.1007/BF02310555 
Crown. (2009). Multi-criteria analysis: a manual. London, England: Government of United Kingdom. Retrieved from

https://www.gov.uk/government/uploads/system/uploads/attachment_data/f ile/7612/1132618.pdf

Daniels, N. (2004). How to Achieve Fair Distribution of ARTs in 3 by 5: Fair Process and Legitimacy in Patient Selection. Retrieved March 31, 2014, from http://www.who.int/ethics/en/background-daniels.pdf

Daniels, N., \& Sabin, J. (1997). Limits to Health Care: Fair Procedures, Democratic Deliberation, and the Legitimacy Problem for Insurers. Philosophy \& Public Affairs, 26(4), 303-350. doi:10.1111/j.1088-4963.1997.tb00082.x

Daniels, N., \& Sabin, J. E. (2000). Accountability for reasonableness. BMJ, 321(7272), 1300-1. doi:10.1136/bmj.a1850

Danziger, S., Montal, R., \& Barkan, R. (2012). Idealistic advice and pragmatic choice: a psychological distance account. Journal of Personality and Social Psychology, 102(6), 1105-1117. doi:10.1037/a0027013

Dean, J. W., \& Sharfman, M. P. (1996). Does Decision Process Matter? A Study of Strategic Decision-Making Effectiveness. The Academy of Management Journal, 39(2), 368-396. Retrieved from http://www.jstor.org/stable/256784

Deas, L., Mattu, L., \& Gnich, W. (2013). Intelligent policy making? Key actors' perspectives on the development and implementation of an early years' initiative in Scotland's public health arena. Social Science \& Medicine, 96, 1-8. doi:http://dx.doi.org/10.1016/j.socscimed.2013.07.001

Deeks, S. G., Lewin, S. R., \& Havlir, D. V. (2013). The end of AIDS: HIV infection as a chronic disease. The Lancet, 382(9903), 1525-1533. doi:10.1016/S01406736(13)61809-7

Defechereux, T., Paolucci, F., Mirelman, A., Youngkong, S., Botten, G., Hagen, T. P., \& Niessen, L. W. (2012). Health care priority setting in Norway a multicriteria decision analysis. BMC Health Services Research, 12, 39. doi:10.1186/1472-6963-12-39

Diaconu, K., Chen, Y.-F., Cummins, C., Jimenez Moyao, G., Manaseki-Holland, S., \& Lilford, R. (2017). Methods for medical device and equipment procurement and prioritization within low- and middle-income countries: findings of a 
systematic literature review. Globalization and Health, 13(1), 59.

doi:10.1186/s12992-017-0280-2

Dixon, J.-G., Gibson, S., McPake, B., \& Maleta, K. (2011). Antiretroviral therapy (ART) rationing and access mechanisms and their impact on youth ART utilization in Malawi. Malawi Medical Journal : The Journal of Medical Association of Malawi, 23(2), 48-54.

Dunbar, R. (2011). How Many Friends Does One Person Need? Dunbar's Number and Other Evolutionary Quirks (p. 312). Cambridge, MA: Harvard University Press.

Edejer, T. T.-T., Baltussen, R., Adam, T., Hutubessy, R., Acharya, A., Evans, D. B., \& Murray, C. J. L. (2003). Making choices in health: WHO guide to costeffectiveness analysis (Vol. 1, p. 329). Geneva, Switzerland: World Health Organization. Retrieved from http://www.who.int/choice/publications/p_2003_generalised_cea.pdf

Emond, M.-O. (2017). Speech of UNAIDS Country Director Ms. Marie-Odile Emond at the Mid-year review meeting of the National AIDS Programme. Retrieved September 27, 2017, from http://www.un.org.vn/en/unaidsspeeches/4468-speech-of-unaids-country-director-ms-marie-odile-emond-atthe-mid-year-review-meeting-of-the-national-aids-programme.html

Forsythe, S., Stover, J., \& Bollinger, L. (2009). The past, present and future of HIV, AIDS and resource allocation. BMC Public Health, 9. doi:10.1186/1471-2458-9S1-S4

Futures Institute. (2013). Spectrum. Retrieved October 10, 2013, from http://www.futuresinstitute.org/spectrum.aspx

Gardner, E. M., McLees, M. P., Steiner, J. F., del Rio, C., \& Burman, W. J. (2011). The Spectrum of Engagement in HIV Care and its Relevance to Test-and-Treat Strategies for Prevention of HIV Infection. Clinical Infectious Diseases, 52(6), 793-800. Retrieved from http://dx.doi.org/10.1093/cid/ciq243

General Statistical Office at the National Institute of Hygiene and Epidemiology/Vietnam and ORC Macro. (2006). Vietnam Population and AIDS Indicator Survey 2005. Calverton, Maryland, USA: GSO, NIHE, and ORC Macro. Retrieved from http://dhsprogram.com/pubs/pdf/AIS3/AIS3.pdf 
Gericke, C. A., Kurowski, C., Ranson, M. K., \& Mills, A. (2005). Intervention complexity--a conceptual framework to inform priority-setting in health. Bulletin of the World Health Organization, 83(4), 285-93. doi:/S004296862005000400013

GFATM. (2012). Global Fund Strategy 2012-16. Retrieved July 26, 2014, from http://www.theglobalfund.org/en/about/strategy/

GFATM. (2013). Guidelines and Requirements for Country Coordinating Mechanisms. Retrieved January 01, 2017, from http://www.theglobalfund.org/documents/ccm/CCM_Requirements_Guideli nes_en

Ghaffar, A., Collins, T., Matlin, S. A., \& Olifson, S. (2009). The 3D Combined Approach Matrix: An Improved Tool for Setting Priorities in Research for Health ( $\mathrm{p}$. 49). Geneva, Switzerland: Global Forum for Health Research. Retrieved from http://www.bvs.hn/Honduras/PIS/MEC3DEnglish.pdf

Glassman, A., Chalkidou, K., Giedion, U., Teerawattananon, Y., Tunis, S., Bump, J. B., \& Pichon-Riviere, A. (2012). Priority-setting institutions in health: Recommendations from a center for global development working group. Global Heart. Geneva, Switzerland: Elsevier. doi:10.1016/j.gheart.2012.01.007

Gliem, J. A., \& Gliem, R. R. (2003). Calculating, Interpreting, And Reporting Cronbach's Alpha Reliability Coefficient For Likert-Type Scales. In Midwest Research to Practice Conference in Adult, Continuing and Community Education (pp. 82-88). Columbus, OH: Ohio State University.

GNP+. (2016). The People Living with HIV Stigma Index. Retrieved October 06, 2016, from http://www.stigmaindex.org/

Goddard, M., Hauck, K., Preker, A., \& Smith, P. C. (2006). Priority setting in health - a political economy perspective. Health Economics, Policy and Law, 1(1), 79 90. doi:10.1017/S1744133105001040

Goodwin, P., \& Wright, G. (2004). Decision Analysis for Management Judgment (3rd ed.). London, England: John Wiley and Sons. Retrieved from http://strathprints.strath.ac.uk/44754/

Graham-Harrison, E. (2016). Global crises overwhelming aid system, says migration chief. Retrieved October 23, 2016, from 
https://www.theguardian.com/global-development/2016/may/14/wardisasters-epidemics-exhaust-aid-resources

Granich, R. M., Gilks, C. F., Dye, C., De Cock, K. M., \& Williams, B. G. (2017). Universal voluntary HIV testing with immediate antiretroviral therapy as a strategy for elimination of HIV transmission: a mathematical model. The Lancet, 373(9657), 48-57. doi:10.1016/S0140-6736(08)61697-9

Green, C., \& Gerard, K. (2009). Exploring the social value of health-care interventions: a stated preference discrete choice experiment. Health Economics, 18(8), 951-976. doi:10.1002/hec.1414

Grin, J., Graaf, H. van der, \& Hoppe, R. (1997). Technology assessment through interaction: a guide (Working do., p. 98). The Hague, The Netherlands: Den Haag: Rathenau Instituut. Retrieved from http://hdl.handle.net/11245/1.129444

Guindo, L. A., Wagner, M., Baltussen, R., Rindress, D., van Til, J., Kind, P., \& Goetghebeur, M. M. (2012). From efficacy to equity: Literature review of decision criteria for resource allocation and healthcare decisionmaking. Cost Effectiveness and Resource Allocation, 10(1), 9. doi:10.1186/1478-7547-10-9

Gwet, K. L. (2007). Intrarater Reliability. In Wiley Encyclopedia of Clinical Trials. John Wiley \& Sons, Inc. doi:10.1002/9780471462422.eoct631

Habermas, J. (1971). Toward a Rational Society: Student Protest, Science, and Politics. (J. J. Shapiro, Trans.) (p. 132). London, England: Heinemann. Retrieved from https://books.google.com.vn/books?id=cW7PmVj7kzQC

Ham, C. (1997). Priority setting in health care: learning from international experience. Health Policy, 42(1), 49-66. doi:https://doi.org/10.1016/S01688510(97)00054-7

Ham, C., \& Coulter, A. (2001). Explicit and implicit rationing: taking responsibility and avoiding blame for health care choices. Journal of Health Services Research \& Policy, 6(3), 163-169. doi:10.1258/1355819011927422

Hanson, K. (2012). Delivering Health Services: incentives and information in supply-side innovations. In R. D. Smith \& K. Hanson (Eds.), Health systems in low-and middle-income countries: An economic and policy perspective (pp. 103124). Oxford: Oxford University Press. 
Hargreaves, J. R. M., Goodman, C., Davey, C., Willey, B. A., Avan, B. I., \& Schellenberg, J. R. A. (2016). Measuring implementation strength: lessons from the evaluation of public health strategies in low- and middle-income settings. Health Policy and Planning, 31(7), 860-867. doi:10.1093/heapol/czw001

Haskins, R., \& Baron, J. (2011). Building the Connection between Policy and Evidence: The Obama evidence-based initiatives. London. Retrieved from https://www.nesta.org.uk

Health Finance \& Governance. (2014). Mobilizing Domestic Funding, Resources to Fight HIV and AIDS. Retrieved July 19, 2016, from https://www.hfgproject.org/mobilizing-domestic-resources-hiv-aids/

Health Metrics Network. (2008). Framework and Standards for Country Health Information Systems. World Health Organization (2nd ed., p. 72). Geneva, Switzerland: World Health Organization. Retrieved from http://apps.who.int/iris/bitstream/10665/43872/1/9789241595940_eng.pdf

Heckathorn, D. D. (1997). Respondent-driven sampling: a new approach to the study of hidden populations. Social Problems, 44(2), 174-199.

Hedayat, A. S., Sloane, N. J. A., \& Stufken, J. (1999). Orthogonal Arrays (1st ed., p. xxiv+416). New York, NY: Springer New York. doi:10.1007/978-1-4612-1478-6

Henriksson, D. K., Ayebare, F., Waiswa, P., Peterson, S. S., Tumushabe, E. K., \& Fredriksson, M. (2017). Enablers and barriers to evidence based planning in the district health system in Uganda; perceptions of district health managers. BMC Health Services Research, 17(1), 103. doi:10.1186/s12913-017-2059-9

Herek, G. M., Capitanio, J. P., \& Widaman, K. F. (2003). Stigma, social risk, and health policy: Public attitudes toward HIV surveillance policies and the social construction of illness. Health Psychology, 22(5), 533-540. doi:10.1037/02786133.22.5.533

Herek, G. M., Kimmel, D. C., Amaro, H., \& Melton, G. B. (1991). Avoiding heterosexist bias in psychological research. American Psychologist, 46(9), 957963. doi:10.1037/0003-066X.46.9.957

Hien, N. T., Long, N. T., \& Huan, T. Q. (2004). HIV/AIDS Epidemics in Vietnam: Evolution and Responses. AIDS Education and Prevention, 16(Supplement A), 137-154. doi:10.1521/aeap.16.3.5.137.35527 
Hipgrave, D. B., Alderman, K. B., Anderson, I., \& Soto, E. J. (2014). Health sector priority setting at meso-level in lower and middle income countries: Lessons learned, available options and suggested steps. Social Science \& Medicine, 102, 190-200. doi:https://doi.org/10.1016/j.socscimed.2013.11.056

Hogan, D. R. (2005). Cost effectiveness analysis of strategies to combat HIV/AIDS in developing countries. BMJ, 331(7530), 1431-1437. doi:10.1136/bmj.38643.368692.68

Horsburgh, D. (2003). Evaluation of qualitative research. Journal of Clinical Nursing, 12(2), 307-312. doi:10.1046/j.1365-2702.2003.00683.x

Hosek, S. G., Siberry, G., Bell, M., Lally, M., Kapogiannis, B., Green, K., ... Wilson, C. M. (2013). The Acceptability and Feasibility of an HIV Preexposure Prophylaxis (PrEP) Trial With Young Men Who Have Sex With Men. JAIDS Journal of Acquired Immune Deficiency Syndromes, 62(4), 447-456. doi:10.1097/QAI.0b013e3182801081

Hsieh, H.-F., \& Shannon, S. E. (2005). Three Approaches to Qualitative Content Analysis. Qualitative Health Research, 15(9), 1277-1288. doi:10.1177/1049732305276687

Hunsmann, M. (2012). Limits to evidence-based health policymaking: Policy hurdles to structural HIV prevention in Tanzania. Social Science and Medicine, 74(10), 1477-1485. doi:10.1016/j.socscimed.2012.01.023

Husain, S., Kadir, M., \& Fatmi, Z. (2007). Resource allocation within the National AIDS Control Program of Pakistan: a qualitative assessment of decision maker's opinions. BMC Health Services Research, 7(1), 11. doi:10.1186/14726963-7-11

IHP+. (2013). Joint Assessment of National Health Strategies (JANS) Tool \& Guidelines. Retrieved November 02, 2014, from http://www.internationalhealthpartnership.net/en/tools/jans-tool-andguidelines/

Innes, J. E., \& Booher, D. E. (2004). Reframing public participation: strategies for the 21st century. Planning Theory \& Practice, 5(4), 419-436. doi:10.1080/1464935042000293170 
Institute of Medicine (US) Committee on Public Health Strategies to Improve Health. (2011). Measurement and Accountability. In For the Public's Health: The Role of Measurement in Action and Accountability. Washington (DC). Retrieved from https:/www.ncbi.nlm.nih.gov/books/NBK209715/

IRMT. (2002). Evidence-Based Governance in the Electronic Age: Building Awareness and Capacity. London, England. Retrieved from http://www.irmt.org/documents/research_reports/project_reports/IRMT_proj _rpt_1-9_2002.pdf

Johansson, K. A., Jerene, D., \& Norheim, O. F. (2008). National HIV treatment guidelines in Tanzania and Ethiopia: are they legitimate rationing tools? Journal of Medical Ethics, 34(6), 478-483. doi:10.1136/jme.2007.021329

Johnston, L. G., Whitehead, S., Simic-Lawson, M., \& Kendall, C. (2010). Formative research to optimize respondent-driven sampling surveys among hard-toreach populations in HIV behavioral and biological surveillance: lessons learned from four case studies. AIDS Care, 22(6), 784-792. doi:10.1080/09540120903373557

Joint United Nations Programme on HIV/AIDS. (1999). From Principle to Practice: Greater Involvement of People Living with or Affected by HIVIAIDS (GIPA). Geneva, Switzerland.

Kabaniha, G. A. (2014). A novel approach to priority-setting for HIV prevention among adults in Uganda. Deakin University. Retrieved from http://hdl.handle.net/10536/DRO/DU:30067354

Kahneman, D. (2011). Thinking, fast and slow (p. 499). New York, NY: Farrar, Straus and Giroux.

Kahneman, D., Rosenfield, A. M., Gandhi, L., \& Blaser, T. (2016). Noise: How to Overcome the of Inconsistent Decision Making. Harvard Business Review, 94(10), 36-43. Retrieved from https://hbr.org/2016/10/noise

Kaló, Z., Bodrogi, J., Boncz, I., Dózsa, C., Jóna, G., Kövi, R., ... Sinkovits, B. (2013). Capacity Building for HTA Implementation in Middle-Income Countries: The Case of Hungary. Value in Health Regional Issues, 2(2), 264-266. doi:http://dx.doi.org/10.1016/j.vhri.2013.06.002 
Kaló, Z., Gheorghe, A., Huic, M., Csanádi, M., \& Kristensen, F. B. (2016). HTA Implementation Roadmap in Central and Eastern European Countries. Health Economics, 25, 179-192. doi:10.1002/hec.3298

Kantrowitz, A. (1975). Views: Controlling Technology Democratically: The paternalistic doctrine of the moral responsibility of science has led to a chaotic situation in which emotional controversy has obscured technical information vital to democratic control of technology. American Scientist, 63(5), 505-509. Retrieved from http://www.jstor.org/stable/27845676

Kapiriri, L., \& Martin, D. K. (2007). A strategy to improve priority setting in developing countries. Health Care Analysis, 15(3), 159-167. doi:10.1007/s10728006-0037-1

Kaufmann, D., Kraay, A., \& Mastruzzi, M. (2013). Worldwide Governance Indicators Project. Retrieved March 20, 2015, from http://www.govindicators.org

Keeney, R. L. (1982). Decision Analysis: An Overview. Operations Research, 30(5), 803-838.

Keeney, R. L., \& Raiffa, H. (1993). Decisions with Multiple Objectives: Preferences and Value Trade-Offs (p. 569). Cambridge, MA: Cambridge University Press.

Kenny, N., \& Joffres, C. (2008). An ethical analysis of international health prioritysetting. Health Care Analysis. Springer. doi:10.1007/s10728-007-0065-5

Keren, G., \& Bruin, W. B. de. (2003). On the Assessment of Decision Quality: Considerations Regarding Utility, Conflict and Accountability. In D. Hardman \& L. Macchi (Eds.), Thinking: Psychological Perspectives on Reasoning, Judgment and Decision Making (pp. 347-363). Chichester, UK: John Wiley \& Sons, Ltd. doi:10.1002/047001332X.ch16

Kerr, N. L., \& Tindale, R. S. (2004). Group Performance and Decision Making. Annual Review of Psychology, 55, 623-655. doi:10.1146/annurev.psych.55.090902.142009

KFF. (2002). Global Spending on HIVIAIDS in Resource-Poor Settings (p. 16). Menlo Park, California. 
KFF, \& UNAIDS. (2016). Financing the Response to AIDS in Low- and Middle-Income Countries: International Assistance from Donor Governments in 2015. Menlo Park, California. Retrieved from http://files.kff.org/attachment/Financing-theResponse-to-HIV-in-Low-and-Middle-Income-Countries-InternationalAssistance-from-Donor-Governments-in-2015

Kilmarx, P. H., \& Mutasa-Apollo, T. (2012). Patching a leaky pipe. Current Opinion in HIV and AIDS, 8(1), 59-64. doi:10.1097/COH.0b013e32835b806e

Klein, R. (2000). From Evidence-Based Medicine to Evidence-Based Policy? Journal of Health Services Research \& Policy, 5(2), 65-66. doi:10.1177/135581960000500201

Koopmanschap, M. A., Stolk, E. A., \& Koolman, X. (2010). Dear policy maker: have you made up your mind? A discrete choice experiment among policy makers and other health professionals. Int J Technol Assess Health Care, 26. doi:10.1017/S0266462310000048

Kumaranayake, L., \& Watts, C. (2001). Resource allocation and priority setting of HIV/AIDS interventions: addressing the generalized epidemic in sub-Saharan Africa. Journal of International Development, 13(4), 451-466. doi:10.1002/jid.797

Larson, E., Vail, D., Mbaruku, G. M., Kimweri, A., Freedman, L. P., \& Kruk, M. E. (2015). Moving Toward Patient-Centered Care in Africa: A Discrete Choice Experiment of Preferences for Delivery Care among 3,003 Tanzanian Women. PLOS ONE, 10(8), e0135621. doi:10.1371/journal.pone.0135621

Lasry, A., Zaric, G. S., \& Carter, M. W. (2007). Multi-level resource allocation for HIV prevention: A model for developing countries. European Journal of Operational Research, 180(2), 786-799. doi:10.1016/j.ejor.2006.02.043

Leelahavarong, P., Teerawattananon, Y., Werayingyong, P., Akaleephan, C., Premsri, N., Namwat, C., ... Tangcharoensathien, V. (2011). Is a HIV vaccine a viable option and at what price? An economic evaluation of adding HIV vaccination into existing prevention programs in Thailand. BMC Public Health, 11(1), 534. doi:10.1186/1471-2458-11-534

Leung, L. (2015). Validity, reliability, and generalizability in qualitative research. Journal of Family Medicine and Primary Care, 4(3), 324. doi:10.4103/22494863.161306 
Leys, M. (2003). Health care policy: qualitative evidence and health technology assessment. Health Policy, 65(3), 217-226. doi:http://dx.doi.org/10.1016/S01688510(02)00209-9

Lim, T., Zelaya, C., Latkin, C., Quan, V. M., Frangakis, C., Ha, T. V., ... Go, V. (2013). Individual-level socioeconomic status and community-level inequality as determinants of stigma towards persons living with HIV who inject drugs in Thai Nguyen, Vietnam. Journal of the International AIDS Society, 16(3 Suppl 2), 18637.

Lopez, A. D., Mathers, C. D., Ezzati, M., Jamison, D. T., \& Murray, C. J. (2006). Measuring the Global Burden of Disease and Risk Factors, 1990-2001. In Global Burden of Disease and Risk Factors (pp. 1-13). Washington, DC: World Bank. Retrieved from http://www.ncbi.nlm.nih.gov/books/NBK11817/

Louviere, J. J., Flynn, T. N., \& Carson, R. T. (2010). Discrete Choice Experiments Are Not Conjoint Analysis. Journal of Choice Modelling, 3(3), 57-72. doi:http://dx.doi.org/10.1016/S1755-5345(13)70014-9

Lovallo, D., \& Sibony, O. (2010). The case for behavioral strategy. McKinsey Quarterly, 2(1), 30-43.

Lussier, M.-T., Richard, C., Bennett, T.-L., Williamson, T., \& Nagpurkar, A. (2012). Surveillance or research: what's in a name? Canadian Family Physician, 58(1), 117.

Luyten, J., Kessels, R., Goos, P., \& Beutels, P. (2015). Public Preferences for Prioritizing Preventive and Curative Health Care Interventions: A Discrete Choice Experiment. Value in Health, 18(2), 224-233. doi:http://dx.doi.org/10.1016/j.jval.2014.12.007

Mabin, V., \& Beattie, M. (2006). A Practical Guide to Multi-Criteria Decision Analysis: A Workbook Companion to V.I.S.A (5th ed., p. 109). Wellington, NZ: Victoria University of Wellington.

Magnani, R., Sabin, K., Saidel, T., \& Heckathorn, D. (2005). Review of sampling hard-to-reach and hidden populations for HIV surveillance. AIDS, 19(Suppl 2), S67-S72.

Mahapatra, P. (2002). Priority setting in the health sector and summary measures of population health. In C. J. L. Murray, J. A. Salomon, C. D. Mathers, \& A. D. 
Lopez (Eds.), Summary Measures of Population Health: Concepts, Ethics, Measurement and Applications (pp. 83-89). Geneva, Switzerland: World Health Organization.

Malhotra, S., \& Zodpey, S. (2010). Operations research in public health. Indian Journal of Public Health, 54(3), 145-150. doi:10.4103/0019-557X.75737

Malyshev, N. (2006). Regulatory Policy: OECD Experience and Evidence. Oxford Review of Economic Policy, 22(2), 274-299. Retrieved from http://dx.doi.org/10.1093/oxrep/grj017

Marcus, U., Hickson, F., Weatherburn, P., \& Schmidt, A. J. (2013). Estimating the size of the MSM populations for 38 European countries by calculating the survey-surveillance discrepancies (SSD) between self-reported new HIV diagnoses from the European MSM internet survey (EMIS) and surveillancereported HIV diagnoses am. BMC Public Health, 13(1), 919. doi:10.1186/14712458-13-919

Marsh, K., IJzerman, M., Thokala, P., Baltussen, R., Boysen, M., Kaló, Z., ... Devlin, N. (2016). Multiple Criteria Decision Analysis for Health Care Decision Making-Emerging Good Practices: Report 2 of the ISPOR MCDA Emerging Good Practices Task Force. Value in Health, 19(2), 125-137. doi:10.1016/j.jval.2015.12.016

Marsh, K., Lanitis, T., Neasham, D., Orfanos, P., \& Caro, J. (2014). Assessing the Value of Healthcare Interventions Using Multi-Criteria Decision Analysis: A Review of the Literature. PharmacoEconomics, 32(4), 345-365. doi:10.1007/s40273-014-0135-0

Marttunen, M. (2011). Interactive multi-criteria decision analysis in the collaborative management of watercourses. Aalto University. Retrieved from https://aaltodoc.aalto.fi/handle/123456789/5035

Matthews, Z., \& Velleman, R. (1997). “New Age” Travellers, Urban Slum Dwellers, Aborigines and Drug Users: Experiences of Collecting Sensitive Data From Marginalised Communities. Bulletin de Méthodologie Sociologique, 57(1), 65-85. doi:10.1177/075910639705700104

McGough, L. J., Reynolds, S. J., Quinn, T. C., \& Zenilman, J. M. (2005). Which Patients First? Setting Priorities for Antiretroviral Therapy Where Resources 
Are Limited. American Journal of Public Health, 95(7), 1173-1180.

doi:10.2105/AJPH.2004.052738

McGregor, S., Henderson, K. J., \& Kaldor, J. M. (2014). How Are Health Research Priorities Set in Low and Middle Income Countries? A Systematic Review of Published Reports. PLoS ONE, 9(10), e108787.

doi:10.1371/journal.pone.0108787

Mentzakis, E., Paolucci, F., \& Rubicko, G. (2014). Priority setting in the Austrian healthcare system: results from a discrete choice experiment and implications for mental health. Health Policy Econ June P, 17(2 SRC - GoogleScholar FG $0), 61-73$.

Merriam, S. B. (1995). What Can You Tell From An N of 1?: Issues of Validity and Reliability in Qualitative Research. PAACE Journal of Lifelong Learning, 4, 5160.

Mertens, D. M. (2009). Research and Evaluation in Education and Psychology:

Integrating Diversity With Quantitative, Qualitative, and Mixed Methods. (V. Knight, Ed.) (3rd ed., p. 527). SAGE Publications. Retrieved from https://books.google.com.vn/books?id=m0N3tcIVds8C

Metz, T. (2008). Respect for persons permits prioritizing treatment for HIV/AIDS. Developing World Bioethics, 8(2), 89-103.

Michaelis, A. P. (2002). Priority-setting ethics in public health. Journal of Public Health Policy, 23(4), 399-412.

Miller, M., Barrett, S., \& Henderson, D. A. (2006). Control and Eradication. In D. T. Jamison, J. G. Breman, A. R. Measham, G. Alleyne, M. Claeson, D. B. Evans, ... P. Musgrove (Eds.), Disease Control Priorities in Developing Countries.

Washington, DC: The International Bank for Reconstruction and Development / The World Bank. Retrieved from http://www.ncbi.nlm.nih.gov/pubmed/21250339

Minh, T. (2016). Deputy PM Vu Duc Dam gave priority to HIV/AIDS prevention. Retrieved March 27, 2017, from http://hanoitimes.com.vn/healtheducation/2016/06/81e0a485/deputy-pm-vu-duc-dam-gave-priority-to-hivaids-prevention/ 
Mirelman, A., Mentzakis, E., Kinter, E., Paolucci, F., Fordham, R., Ozawa, S., ... Niessen, L. W. (2012). Decision-Making Criteria among National Policymakers in Five Countries: A Discrete Choice Experiment Eliciting Relative Preferences for Equity and Efficiency. Value in Health, 15(3), 534-539. doi:10.1016/j.jval.2012.04.001

Moatti, J. P., Marlink, R., Luchini, S., \& Kazatchkine, M. (2008). Universal access to HIV treatment in developing countries: going beyond the misinterpretations of the "cost-effectiveness" algorithm. AIDS (London, England), 22 Suppl 1, S5966. doi:10.1097/01.aids.0000327624.69974.41

Monnikhof, R. A. H., \& Bots, P. W. G. (2000). On the Application of MCDA in Interactive Spatial Planning Processes: Lessons Learnt from Two Stories from the Swamp. Journal of Multi-Criteria Decision Analysis, 9, 28-44. doi:10.1002/1099-1360(200001/05)9:1/3<28::AID-MCDA265>3.0.CO;2-R

Montaner, J. S. G., Lima, V. D., Barrios, R., Yip, B., Wood, E., Kerr, T., ... Kendall, P. (2010). Association of highly active antiretroviral therapy coverage, population viral load, and yearly new HIV diagnoses in British Columbia, Canada: a population-based study. The Lancet, 376(9740), 532-539. doi:10.1016/S0140-6736(10)60936-1

Montorzi, G., Haan, S. de, \& IJsselmuiden, C. (2010). Priority Setting for Research for Health: a management process for countries. Council on Health Research for Development (COHRED).

Moon, B. K. (2016). Secretary-General's remarks at Back to the Present: Launch of a Time Machine Installation on Data for Children. Retrieved June 01, 2017, from https://www.un.org/sg/en/content/sg/statement/2016-09-14/secretarygenerals-remarks-back-present-launch-time-machine

Mooney, G. H. (1987). What does equity in health mean? World Health Statistics Quarterly, 40(4), 296-303. Retrieved from http://www.ncbi.nlm.nih.gov/pubmed/3433797

Murray, C. J. L., Vos, T., Lozano, R., Naghavi, M., Flaxman, A. D., Michaud, C., ... Memish, Z. A. (2012). Disability-adjusted life years (DALYs) for 291 diseases and injuries in 21 regions, 1990-2010: a systematic analysis for the Global Burden of Disease Study 2010. Lancet, 380(9859), 2197-2223. doi:10.1016/S0140-6736(12)61689-4 
Musgrove, P. (1999). Public spending on health care: how are different criteria related? Health Policy, 47(3), 207-23. Retrieved from http://www.ncbi.nlm.nih.gov/pubmed/10538919

Nadol, P. (2012). Size Estimation: MSM in Vietnam. Retrieved June 02, 2015, from http://www.aidstar-one.com/sites/default/files/6_Nadol_29.pdf

National Committee for AIDS Drug and Prostitution Prevention and Control. National Strategy on HIV/AIDS Prevention and Control Till 2020 with a Vision to 2030 (2012). Hanoi, Viet Nam: Nguyen Xuan Phuc (Prime Minister) - Socialist Republic of Viet Nam.

Nelkin, D. (1981). Wisdom, Expertise, and the Application of Ethics. Science, Technology \& Human Values, 6(1), 16-17.

Neumann, P. J. (2004). Using cost-effectiveness analysis to improve health care: opportunities and barriers. Oxford: Oxford University Press.

Newdick, C. (2005). Accountability for rationing - theory into practice. The Journal of Law, Medicine E Ethics, 33(4), 660-8. Retrieved from http://www.ncbi.nlm.nih.gov/pubmed/16686237

Newman, P. A., Cameron, M. P., Roungprakhon, S., Tepjan, S., \& Scarpa, R. (2016). Acceptability and Preferences for Hypothetical Rectal Microbicides among a Community Sample of Young Men Who Have Sex with Men and Transgender Women in Thailand: A Discrete Choice Experiment. AIDS and Behavior, 20(11), 2588-2601. doi:10.1007/s10461-015-1258-9

Niëns, L. (2014). Affordability in Health Care: Operationalizations and Applications in Different Contexts. Erasmus University Rotterdam. Retrieved from http://hdl.handle.net/1765/50314

Nutbeam, D. (2004). Getting evidence into policy and practice to address health inequalities. Health Promotion International, 19(2), 137-140. Retrieved from http://dx.doi.org/10.1093/heapro/dah201

NYPL. (2015). Why We Fight: Remembering AIDS Activism. Retrieved September 12, 2016, from https://www.nypl.org/events/exhibitions/why-we-fight/more

O’Haire, C., McPheeters, M., Nakamoto, E., LaBrant, L., Most, C., Lee, K., ... Guise, J.-M. (2011). Engaging Stakeholders To Identify and Prioritize Future 
Research Needs. Methods Future Research Needs Reports, No. 4. Rockville, MD: Agency for Healthcare Research and Quality (AHRQ). Retrieved from http://www.ncbi.nlm.nih.gov/pubmed/21977526

Oberth, G., \& Whiteside, A. (2016). What does sustainability mean in the HIV and AIDS response? African Journal of AIDS Research : AJAR, 15(1), 35-43. doi:10.2989/16085906.2016.1138976

OECD. (2011). 2011 DAC Report on Multilateral Aid. Paris, France. Retrieved from https://www.oecd.org/dac/aid-architecture/49014277.pdf

Okello, D., Chongtrakul, P., \& The COHRED Working Group on Priority Setting. (2000). A Manual for Research Priority Setting Using the ENHR Strategy (p. 48). The Council on Health Research for Development (COHRED). Retrieved from http://www.cohred.org/downloads/578.pdf

Oliver, K., Innvar, S., Lorenc, T., Woodman, J., \& Thomas, J. (2014). A systematic review of barriers to and facilitators of the use of evidence by policymakers. BMC Health Services Research, 14(1), 2. doi:10.1186/1472-6963-14-2

Örtengren, K. (2004). The Logical Framework Approach: A summary of the theory behind the LFA method. Stockholm: Sida. Retrieved from http://unpan1.un.org/intradoc/groups/public/documents/un/unpan032558.pd $\mathrm{f}$

Parker, R., \& Aggleton, P. (2003). HIV and AIDS-related stigma and discrimination: a conceptual framework and implications for action. Social Science $\mathcal{E}$ Medicine (1982), 57(1), 13-24.

Peacock, S., Mitton, C., Bate, A., McCoy, B., \& Donaldson, C. (2009). Overcoming barriers to priority setting using interdisciplinary methods. Health Policy, 92(2-3), 124-32. doi:10.1016/j.healthpol.2009.02.006

Peersman, G., Rugg, D., Erkkola, T., Kiwango, E., \& Yang, J. (2009). Are the Investments in National HIV Monitoring and Evaluation Systems Paying Off? JAIDS Journal of Acquired Immune Deficiency Syndromes, 52(Suppl 2), S87S96. doi:10.1097/QAI.0b013e3181baede7

PEPFAR. (2008). PEPFAR's Five-Year Strategy. Retrieved July 26, 2014, from http://www.pepfar.gov/documents/organization/133035.pdf 
PEPFAR. (2015). PEPFAR Country/Regional Operational Plan (COP/ROP) 2015 Guidance. Washington DC: U.S. Department of State. Retrieved from http://www.pepfar.gov/documents/organization/237669.pdf

Pérez, I. J., Wikström, R., Mezei, J., Carlsson, C., \& Herrera-Viedma, E. (2013). A new consensus model for group decision making using fuzzy ontology. Soft Computing, 17(9), 1617-1627. doi:10.1007/s00500-012-0975-5

Pfeffer, J., \& Sutton, R. I. (2006, January). Evidence-Based Management. Harvard Business Review. Retrieved from https://hbr.org/2006/01/evidence-basedmanagement

Pham, Q. D., Wilson, D. P., Kerr, C. C., Shattock, A. J., Do, H. M., Duong, A. T., .. Zhang, L. (2015). Estimating the Cost-Effectiveness of HIV Prevention Programmes in Vietnam, 2006-2010: A Modelling Study. PLOS ONE, 10(7), e0133171. Retrieved from http://dx.doi.org/10.1371\%2Fjournal.pone.0133171

Polit, D. F., \& Beck, C. T. (2010). Generalization in quantitative and qualitative research: Myths and strategies. International Journal of Nursing Studies, 47(11), 1451-1458. doi:10.1016/j.ijnurstu.2010.06.004

R Core Team. (2013). R: A Language and Environment for Statistical Computing. Vienna, Austria: R Foundation for Statistical Computing.

Rankin, K., Heard, A., \& Diaz, N. (2016). Adolescent sexual and reproductive health: Scoping the impact of programming in low- and middle-income countries. New Delhi, India. Retrieved from http://www.3ieimpact.org/media/filer_public/2017/01/18/sp5-asrh.pdf

Ravindran, A. R. (2008). Operations Research Methodologies (p. 476). Boca Raton, FL: CRC Press.

Ravindran, T. S., \& Kelkar-Khambete, A. (2007). Women's health policies and programmes and gender-mainstreaming in health policies, programmes and within health sector institutions. Retrieved March 31, 2014, from http://www.who.int/social_determinants/resources/womens_health_policies_ wgkn_2007.pdf?ua=1

Raymond, A., Hill, A., \& Pozniak, A. (2014). Large disparities in HIV treatment cascades between eight European and high-income countries - analysis of 
break points. Journal of the International AIDS Society, 17(4(Suppl 3)), 19507. doi:10.7448/IAS.17.4.19507

Rayner, S. (2003). Who's in Charge? Worldwide Displacement of Democratic Judgment by Expert Assessments. Economic and Political Weekly, 38(48), 51135119. Retrieved from http://www.jstor.org/stable/4414350

RCore, T. (2013). R: A language and environment for statistical computing. $R$ Foundation for Statistical Computing, Vienna, Austria. Online: Http://www. RProject. Org.

Rehle, T. M., Saidel, T. J., Hassig, S. E., Bouey, P. D., Gaillard, E. M., \& Sokal, D. C. (1998). AVERT: a user-friendly model to estimate the impact of HIV/sexually transmitted disease prevention interventions on HIV transmission. AIDS, 12(Suppl 2), S27-35. Retrieved from

http://www.ncbi.nlm.nih.gov/pubmed/9792359

Richter, A., Hicks, K. A., Earnshaw, S. R., \& Honeycutt, A. A. (2008). Allocating HIV prevention resources: a tool for state and local decision making. Health Policy (Amsterdam, Netherlands), 87(3), 342-349.

doi:10.1016/j.healthpol.2008.01.008

Rivlin, A. M. (1971). Systematic Thinking for Social Action (p. 142). Washington DC: Brookings Institution Press.

Rosen, S., Sanne, I., Collier, A., \& Simon, J. L. (2005a). Hard choices: rationing antiretroviral therapy for HIV/AIDS in Africa. Lancet, 365(9456), 354-356. doi:10.1016/S0140-6736(05)17792-7

Rosen, S., Sanne, I., Collier, A., \& Simon, J. L. (2005b). Rationing antiretroviral therapy for HIV/AIDS in Africa: choices and consequences. PLoS Medicine, 2(11), e303. doi:10.1371/journal.pmed.0020303

Rousson, V., Gasser, T., \& Seifert, B. (2002). Assessing intrarater, interrater and test-retest reliability of continuous measurements. Statistics in Medicine, 21(22), 3431-3446. doi:10.1002/sim.1253

Roy, C. M., \& Cain, R. (2001). The involvement of people living with HIV/AIDS in community-based organizations: Contributions and constraints. AIDS Care, 13(4), 421-432. doi:10.1080/09540120120057950 
Rudan, I., Kapiriri, L., Tomlinson, M., Balliet, M., Cohen, B., \& Chopra, M. (2010). Evidence-Based Priority Setting for Health Care and Research: Tools to Support Policy in Maternal, Neonatal, and Child Health in Africa. PLOS Medicine, 7(7), e1000308. doi:10.1371/journal.pmed.1000308

Sabik, L. M., \& Lie, R. K. (2008). Priority setting in health care: Lessons from the experiences of eight countries. International Journal for Equity in Health, 7(1), 4. doi:10.1186/1475-9276-7-4

Safarnejad, A., Nga, N. T., \& Son, V. H. (2017). Population Size Estimation of Men Who Have Sex with Men in Ho Chi Minh City and Nghe An Using Social App Multiplier Method. Journal of Urban Health, 94(3), 339-349. doi:10.1007/s11524-016-0123-0

Sanderson, I. (2009). Using Evaluation in Policy Practice: Towards "Intelligent Policy Making"in Scotland.

Scheer, L., Garcia, E., Laan, A. L., Burg, S., \& Boenink, M. (2014). The Benefits of Patient Involvement for Translational Research. Health Care Analysis, 1-17. doi:10.1007/s10728-014-0289-0

Schwartländer, B., Stover, J., Hallett, T., Atun, R., Avila, C., Gouws, E., ... Padian, N. (2011). Towards an improved investment approach for an effective response to HIV/AIDS. The Lancet, 377(9782), 2031-2041. doi:10.1016/S01406736(11)60702-2

Shafir, E., Simonson, I., \& Tversky, A. (1993). Reason-based choice. Cognition, 49(12), 11-36. doi:https://doi.org/10.1016/0010-0277(93)90034-S

Shrout, P. E., \& Fleiss, J. L. (1979). Intraclass correlations: Uses in assessing rater reliability. Psychological Bulletin, 86(2), 420-428. doi:http://dx.doi.org/10.1037/0033-2909.86.2.420

Shukla, V., Auriol, G., \& Hipel, K. W. (2016). Multicriteria Decision-Making Methodology for Systems Engineering. IEEE Systems Journal, 10(1), 4-14. doi:10.1109/JSYST.2014.2344635

Sidibé, M., Piot, P., \& Dybul, M. (2012). AIDS is not over. The Lancet, 380(9859), 2058-2060. doi:10.1016/S0140-6736(12)62088-1 
Smith, K. (2013). Beyond Evidence-Based Policy in Public Health: The Interplay of Ideas (1st Editio.). Edinburgh, UK: Palgrave Macmillan UK. doi:10.1057/9781137026583

Söderbaum, P. (2006). Democracy and sustainable development--what is the alternative to cost-benefit analysis? Integrated Environmental Assessment and Management, 2(2), 182-90. Retrieved from http://www.ncbi.nlm.nih.gov/pubmed/16646387

Somekh, B., \& Lewin, C. (2004). Research Methods in the Social Sciences (p. 376). SAGE Publications. Retrieved from https://books.google.com.vn/books?id=Trfg5iWB22MC

Spencer, L., \& Pahl, R. E. (2006). Rethinking Friendship: Hidden Solidarities Today (illustrate., p. 306). Princeton, NJ: Princeton University Press.

Spicer, N., Aleshkina, J., Biesma, R., Brugha, R., Caceres, C., \& Chilundo, B. (2010). National and subnational HIV/AIDS coordination: are global health initiatives closing the gap between intent and practice? Globalization and Health. March 2, 6(3 SRC - GoogleScholar FG - 0).

Stansfield, S. K., Walsh, J., Prata, N., \& Evans, T. (2006). Information to Improve Decision Making for Health. In Disease Control Priorities in Developing Countries (pp. 1017-1030). Washington, DC: World Bank.

Steele, K., Carmel, Y., Cross, J., \& Wilcox, C. (2009). Uses and misuses of multicriteria decision analysis (MCDA) in environmental decision making. Risk Analysis, 29(1), 26-33. doi:10.1111/j.1539-6924.2008.01130.x

Sustainable Cities Programme, United Nations Human Settlements Programme, \& United Nations Environment Programme. (2002). Sustainable Cities Programme, 1990-2000: A Decade of United Nations Support for Broad-based Participatory Management of Urban Development (p. 47). UN-HABITAT.

Tavakol, M., \& Dennick, R. (2011). Making sense of Cronbach's alpha. International Journal of Medical Education, 2, 53-55. doi:10.5116/ijme.4dfb.8dfd

Terwindt, F., Rajan, D., \& Soucat, A. (2016). Priority-setting for national health policies, strategies and plans. In Strategizing national health in the 21st century: a handbook. Geneva, Switzerland: World Health Organization. 
The Global Fund. (2009). Performance-based Funding at the Global Fund. Geneva, Switzerland: The Global Fund to Fight AIDS, Tuberculosis and Malaria.

Retrieved from

http://www.theglobalfund.org/documents/performance/Performance_Perfor manceBasedFunding_Brochure_en/

The Global Fund. (2015). Capacity Assessment Tool User Guide. Geneva,

Switzerland: The Global Fund to Fight AIDS, Tuberculosis and Malaria.

Retrieved from

http://www.theglobalfund.org/documents/core/guidelines/Core_CapacityAss essmentTool_UserGuide_en/

The Government of the Socialist Republic of Viet Nam. Decision of the Prime Minister approving the National Strategy on HIV/AIDS prevention and control in Viet Nam till 2010 with a vision to 2020 (2004). Hanoi, Viet Nam: Government of Viet Nam.

The Government of the Socialist Republic of Viet Nam. (2006). Second Country Report on Following up to the Declaration of Commitment on HIVIAIDS (p. 48). Hanoi, Viet Nam.

The Kaiser Family Foundation, \& UNAIDS. (2013). Financing the Response to HIV in Low- and Middle-Income Countries: International Assistance from Donor Governments in 2012. Geneva, Switzerland.

The World Bank. (2007). Planning and managing for HIVIAIDS results : a handbook. Washington DC. Retrieved from http://documents.worldbank.org/curated/en/2007/09/9095764/planningmanaging-hivaids-results-handbook

The World Bank. (2011). The World Bank and HIV/AIDS. Retrieved July 26, 2014, from http://web.worldbank.org/WBSITE/EXTERNAL/TOPICS/EXTHEALTHNUTR ITIONANDPOPULATION/EXTHIVAIDS/0,,contentMDK:23053804 menuPK :4896254 pagePK:210058 piPK:210062 theSitePK:376471,00.html

Thirty years of a disease: The end of AIDS? (2011, June). The Economist. Retrieved from http://www.economist.com/node/18774722 
Tickner, J. A., \& Wright, S. (2003). The precautionary principle and democratizing expertise: a US perspective. Science and Public Policy, 30(3), 213-218. doi:10.3152/147154303781780470

Tromp, N., \& Baltussen, R. (2012). Mapping of multiple criteria for priority setting of health interventions: an aid for decision makers. BMC Health Services Research, 12(1), 454. doi:10.1186/1472-6963-12-454

Tromp, N., Prawiranegara, R., Siregar, A., Jansen, M. P. M., \& Baltussen, R. (2016). Time to recognise countries' preferences in HIV control. The Lancet, 387(10023), 1053-1054. doi:10.1016/S0140-6736(16)00659-0

Tromp, N., Prawiranegara, R., Siregar, A., Sunjaya, D., \& Baltussen, R. (2015). IMPORTANCE OF MULTIPLE CRITERIA FOR PRIORITY SETTING OF HIV/AIDS INTERVENTIONS. International Journal of Technology Assessment in Health Care, 31(6), 390-398. doi:10.1017/S0266462316000039

Tromp, N., Prawiranegara, R., Subhan Riparev, H., Siregar, A., Sunjaya, D., \& Baltussen, R. (2015). Priority setting in HIV/AIDS control in West Java Indonesia: an evaluation based on the accountability for reasonableness framework. Health Policy and Planning, 30(3), 345-355.

doi:10.1093/heapol/czu020

Tuan, L. A., Tuan, N. A., Thanh, D. C., Quang, T. D., \& Sabin, K. (2014). Multiple approaches to population size estimation of injecting drug users (IDU), female sex workers (FSW) and men who have sex with men (MSM) in three Vietnamese provinces. In 19th International AIDS Conference (p. Abstract no. TUPE197). Melbourne, Australia: IAS.

Tukey, J. W. (1977). Exploratory Data Analysis. Reading, PA: Addison-Wesley Publishing Company. Retrieved from https://books.google.com.vn/books?id=UT9dAAAAIAAJ

UN. (2011). Political Declaration on HIV/AIDS: Intensifying our Efforts to Eliminate HIVIAIDS. New York: UNGA.

UNAIDS. (1998). Guide to the Strategic Planning Process for a National Response to HIV/AIDS. Geneva: UNAIDS. Retrieved from http://data.unaids.org/publications/irc-pub01/jc267-stratplan1_en.pdf 
UNAIDS. (2004a). Consultation on Harmonization of International AIDS Funding. Retrieved March 28, 2015, from http://data.unaids.org/UNA-docs/ThreeOnes_Agreement_en.pdf

UNAIDS. (2004b). Consultation on Harmonization of International AIDS Funding. Washington DC. Retrieved from http://data.unaids.org/UNA-docs/ThreeOnes_Agreement_en.pdf

UNAIDS. (2004c). Report on the Global AIDS Epidemic (p. 21). Geneva, Switzerland.

UNAIDS. (2004d). “Three Ones” key principles. Washington DC: UNAIDS. Retrieved from http://data.unaids.org/UNA-docs/ThreeOnes_KeyPrinciples_en.pdf

UNAIDS. (2008). A framework for monitoring and evaluating HIV prevention programmes for most-at-risk populations (p. 91). Geneva, Switzerland: Joint United Nations Programme on HIV/AIDS. Retrieved from http://www.unaids.org/sites/default/files/sub_landing/17_Framework_ME_Pr evention_Prog_MARP_E.pdf

UNAIDS. (2010a). Strategic Guidance for Evaluating HIV Prevention Programmes. Geneva, Switzerland: Joint United Nations Programme on HIV/AIDS.

UNAIDS. (2010b). UNAIDS Strategy 2011-2015. Retrieved March 30, 2014, from http://www.unaids.org/en/media/unaids/contentassets/documents/unaidspu blication/2010/JC2034_UNAIDS_Strategy_en.pdf

UNAIDS. (2013a). Global Report: UNAIDS Report on the Global AIDS Epidemic 2013. Geneva, Switzerland.

UNAIDS. (2013b). Smart Investments. (p. 27). Geneva, Switzerland.

UNAIDS. (2015). AIDSinfo. Retrieved August 26, 2016, from http://aidsinfo.unaids.org/

UNAIDS. (2016a). 90-90-90: On the right track towards the global target (p. 58). Geneva, Switzerland.

UNAIDS. (2016b). AIDS by the Numbers. Geneva, Switzerland. 
UNAIDS. (2016c). Fast-Track Cities: Cities Ending the AIDS Epidemic (p. 61). Geneva, Switzerland.

UNAIDS. (2016d). Fast-Track Update on Investments Needed in the AIDS Response (p. 20). Geneva, Switzerland. Retrieved from http://www.unaids.org/sites/default/files/media_asset/UNAIDS_Reference_F astTrack_Update_on_investments_en.pdf

UNAIDS. (2016e). Global AIDS Update (p. 13). Geneva, Switzerland.

UNAIDS. (2016f). Kaiser/UNAIDS Study Finds Donor Government Funding for HIV Fell in 2015 for First Time in 5 Years. Retrieved February 26, 2017, from http://www.unaids.org/en/resources/presscentre/pressreleaseandstatementar chive/2016/july/20150815_kaiser

UNAIDS, IMPACT, \& FHI. (2003). Estimating the Size of Populations at Risk for HIV: Issues and Methods. (E. Pisani, Ed.) (p. 69). Arlington, VA: Family Health International.

UNAIDS, \& WHO. (2010). Guidelines on Estimating the Size of Populations Most at Risk to HIV (p. 51). Geneva, Switzerland: World Health Organization.

UNDG. (2003). Guidance Note on Joint Programming. New York: United Nations Development Group. Retrieved from http://www.who.int/hdp/publications/5d.pdf

UNDP. (2015). Preventing HIV Transmission in Intimate Partner Relationships: Evidence, strategies and approaches for addressing concentrated HIV epidemics in Asia. Bangkok, Thailand. Retrieved from http://www.aidsdatahub.org/sites/default/files/publication/rbap-hhd-2015preventing-hiv-transmission-in-intimate-partner-relationships.pdf

United Nations Development Programme, \& Bureau for Development Policy. (2011). Official Development Assistance. In L. Garmer (Ed.), Towards Human Resilience: Sustaining MDG Progress in an Age of Economic Uncertainty (pp. 144183). New York: UNDP. Retrieved from http://www.undp.org/content/dam/undp/library/Poverty Reduction/Towards_SustainingMDG_Web1005.pdf 
USGAO. (1996). Content Analysis: A Methodology for Structuring and Analyzing Written Material. Washington DC: United States General Accounting Office. Retrieved from http://www.gao.gov/assets/80/76281.pdf

Vadivoo, S., Gupte, M. D., Adhikary, R., Kohli, A., Kangusamy, B., Joshua, V., ... Goswami, P. (2008). Appropriateness and execution challenges of three formal size estimation methods for high-risk populations in India. AIDS, 22(Suppl 5), S137-S148. doi:10.1097/01.aids.0000343772.82647.2d

Van de Schoot, T., Pavlova, M., Atanasova, E., \& Groot, W. (2017). Preferences of Bulgarian consumers for quality, access and price attributes of healthcare services-result of a discrete choice experiment. The International Journal of Health Planning and Management, 32(1), e47-e71. doi:10.1002/hpm.2325

Vandepitte, J., Lyerla, R., Dallabetta, G., Crabbé, F., Alary, M., \& Buvé, A. (2006). Estimates of the number of female sex workers in different regions of the world. Sexually Transmitted Infections, 82(suppl 3), iii18-iii25.

Verguet, S. (2013). Efficient and equitable HIV prevention: A case study of male circumcision in South Africa. Cost Effectiveness and Resource Allocation, 11(1), 1. doi:10.1186/1478-7547-11-1

Viergever, R. F., Olifson, S., Ghaffar, A., \& Terry, R. F. (2010). A checklist for health research priority setting: nine common themes of good practice. Health Research Policy and Systems, 8, 36. doi:10.1186/1478-4505-8-36

Viet Nam Administration of HIV/AIDS Control. (2007). National Monitoring and Evaluation Framework for HIV Prevention and Control Programs. Hanoi, Viet Nam: Ministry of Health, Viet Nam Administration of HIV/AIDS Control (VAAC).

Viet Nam Ministry of Health. (2007). Program of Action on HIV/AIDS surveillance, monitoring and evaluation (p. 38). Hanoi, Viet Nam.

Viet Nam Ministry of Health. (2014). Optimizing Viet Nam's HIV response: an investment case. Hanoi, Viet Nam. Retrieved from http://www.unaids.org/sites/default/files/country/documents/VNM_narrative _report_2015.pdf

Vietnam Authority of HIV/AIDS Control. (2013). Mid-term Review of the Targets of the Political Declaration on HIV. Hanoi. 
Vietnam Ministry of Health. (2006). Scaling up Towards Universal Access to HIVIAIDS Prevention, Treatment, Care and Support in Viet Nam (p. 64). Hanoi, Viet Nam.

Vietnam Ministry of Health. (2016). Joint Annual Health Review 2015: Strengthening primary health care at the grassroots towards universal health coverage. Hanoi, Viet Nam. Retrieved from http://jahr.org.vn/downloads/JAHR2015/JAHR2015_full_EN.pdf

Vietnam Ministry of Planning and Investment. (2015). Country Report: 15 Years of Achieving the Viet Nam Millenium Development Goals. Hanoi, Viet Nam.

Vietnam Ministry of Planning and Investment, \& UNFPA. (2010). The 2009 Vietnam Population and Housing Census: Major Findings (p. 508). Hanoi, Viet Nam.

Vlek, C. (1984). What constitutes "a good decision"? Acta Psychologica, 56(1-3), 5-27. doi:10.1016/0001-6918(84)90004-0

Vogel, J. P., Oxman, A. D., Glenton, C., Rosenbaum, S., Lewin, S., Gulmezoglu, A. M., \& Souza, J. P. (2013). Policymakers' and other stakeholders' perceptions of key considerations for health system decisions and the presentation of evidence to inform those considerations: an international survey. Health Research Policy and Systems, 11(19), 1-9. doi:10.1186/1478-4505-11-19

Vuorenkoski, L., Toiviainen, H., \& Hemminki, E. (2008). Decision-making in priority setting for medicines--a review of empirical studies. Health Policy, 86. doi:10.1016/j.healthpol.2007.09.007

Waite, K. R., Paasche-Orlow, M., Rintamaki, L. S., Davis, T. C., \& Wolf, M. S. (2008). Literacy, social stigma, and HIV medication adherence. Journal of General Internal Medicine, 23(9), 1367-1372. doi:10.1007/s11606-008-0662-5

Walensky, R. P., Wood, R., Ciaranello, A. L., Paltiel, A. D., Lorenzana, S. B., Anglaret, X., ... Investigators, for the C.-I. (2010). Scaling Up the 2010 World Health Organization HIV Treatment Guidelines in Resource-Limited Settings: A Model-Based Analysis. PLOS Medicine, 7(12), e1000382. Retrieved from http://dx.doi.org/10.1371\%2Fjournal.pmed.1000382

Weber, R. P. (1990). Basic content analysis. (S. MrElroy, Ed.) (2nd Editio.). Newbury Park, CA: Sage Publications. doi:http://dx.doi.org/10.4135/9781412983488 
WEF. (2014). The Global Competitiveness Report 2014-2015. Geneva, Switzerland. Retrieved from http://reports.weforum.org/global-competitiveness-report2014-2015/

Wejnert, C., \& Heckathorn, D. D. (2008). Web-Based Network Sampling. Sociological Methods \& Research, 37(1), 105-134. doi:10.1177/0049124108318333

Wenstøp, F., \& Magnus, P. (2001). Value focused rationality in AIDS policy. Health Policy, 57(1), 57-72. Retrieved from http://www.ncbi.nlm.nih.gov/pubmed/11348694

WHO. (2010). A Framework for National Health Policies, Strategies and Plans. Geneva: World Health Organization. Retrieved from http://www.who.int/nationalpolicies/FrameworkNHPSP_final_en.pdf

WHO. (2011). Causes of death by age, sex and region, 2000-2011. Retrieved March 31, 2014, from http://www.who.int/healthinfo/global_burden_disease/estimates_regional/en /

WHO. (2013a). Consolidated Guidelines on the use of Antiretroviral Drugs for Treating and Preventing HIV Infection. Geneva, Switzerland.

WHO. (2013b). Health 2020: A European policy framework and strategy for the 21st century (p. 182). Geneva. Retrieved from http://www.euro.who.int/_data/assets/pdf_file/0011/199532/Health2020Long.pdf?ua=1

WHO. (2014a). Consolidated Guidelines on HIV Prevention, Diagnosis, Treatment and Care for Key Populations (p. 159). Geneva, Switzerland: World Health Organization.

WHO. (2014b). Policy brief: Consolidated guidelines on HIV prevention, diagnosis, treatment and care for key populations. Geneva, Switzerland.

Woodford, M. R., \& Preston, S. (2013). Strengthening Citizen Participation in Public Policy-Making: A Canadian Perspective. Parliamentary Affairs, 66(2), 345-363. Retrieved from http://dx.doi.org/10.1093/pa/gsr065

World Bank. (2013). Vietnam: Achieving Success as a Middle-income Country. Retrieved August 28, 2016, from 
http://www.worldbank.org/en/results/2013/04/12/vietnam-achieving-successas-a-middle-income-country

World Health Organization. (2003). The 3 by 5 Initiative. Retrieved July 26, 2014, from http://www.who.int/3by5/en/

World Health Organization. (2007). Everybody's business : strengthening health systems to improve health outcomes: WHO's framework for action (p. 44). Geneva, Switzerland: World Health Organization. Retrieved from http://www.who.int/healthsystems/strategy/everybodys_business.pdf

World Health Organization. (2017). Ten years in public health, 2007-2017: report by Dr Margaret Chan, Director-General, World Health Organization. Geneva, Switzerland. Retrieved from http://apps.who.int/iris/bitstream/10665/255355/1/9789241512442eng.pdf?ua $=1$

Wye, L., Brangan, E., Cameron, A. M., Gabbay, J., Klein, J. H., \& Pope, C. J. (2015). Evidence based policy making and the "art" of commissioning - how English healthcare commissioners access and use information and academic research in "real life" decision-making. BMC Health Services Research, 15(430). doi:10.1186/s12913-015-1091-x

Xu, L., \& Yang, J.-B. (2001). Introduction to Multi-Criteria Decision Making and the Evidential Reasoning Approach (No. 0106) (p. 21). Manchester, UK. Retrieved from https://phps.portals.mbs.ac.uk/Portals/49/docs/jyang/XuYang_MSM_Workin gPaperFinal.pdf

Yamin, A. E. (2008). Beyond Compassion: The Central Role of Accountability in Applying a Human Rights Framework to Health. Health and Human Rights, 10(2), 1-20. Retrieved from http://www.jstor.org/stable/20460100

Youngkong, S., Baltussen, R., Tantivess, S., Koolman, X., \& Teerawattananon, Y. (2010). Criteria for priority setting of HIV/AIDS interventions in Thailand: a discrete choice experiment. BMC Health Services Research, 10(1), 197. doi:10.1186/1472-6963-10-197

Youngkong, S., Kapiriri, L., \& Baltussen, R. (2009). Setting priorities for health interventions in developing countries: A review of empirical studies. Tropical 
Medicine and International Health, 14(8), 930-939. doi:10.1111/j.13653156.2009.02311.x

Yu, D., Calleja, J. M. G., Zhao, J., Reddy, A., \& Seguy, N. (2014). Estimating the size of key populations at higher risk of HIV infection: a summary of experiences and lessons presented during a technical meeting on size estimation among key populations in Asian countries. Western Pacific Surveillance and Response, 5(3), 43-49. doi:10.5365/wpsar.2014.5.2.008

Zhang, L., Maher, L., Pham, Q. D., Higgs, P., Anh, N. D., Duc, B. H., ... Wilson, D. P. (2012). Evaluation of a decade of DFID and World Bank supported HIV and AIDS programmes in Vietnam from 2003 to 2012. Sydney, Australia. Retrieved from http://optimamodel.com/pubs/vietnam-report2.pdf 


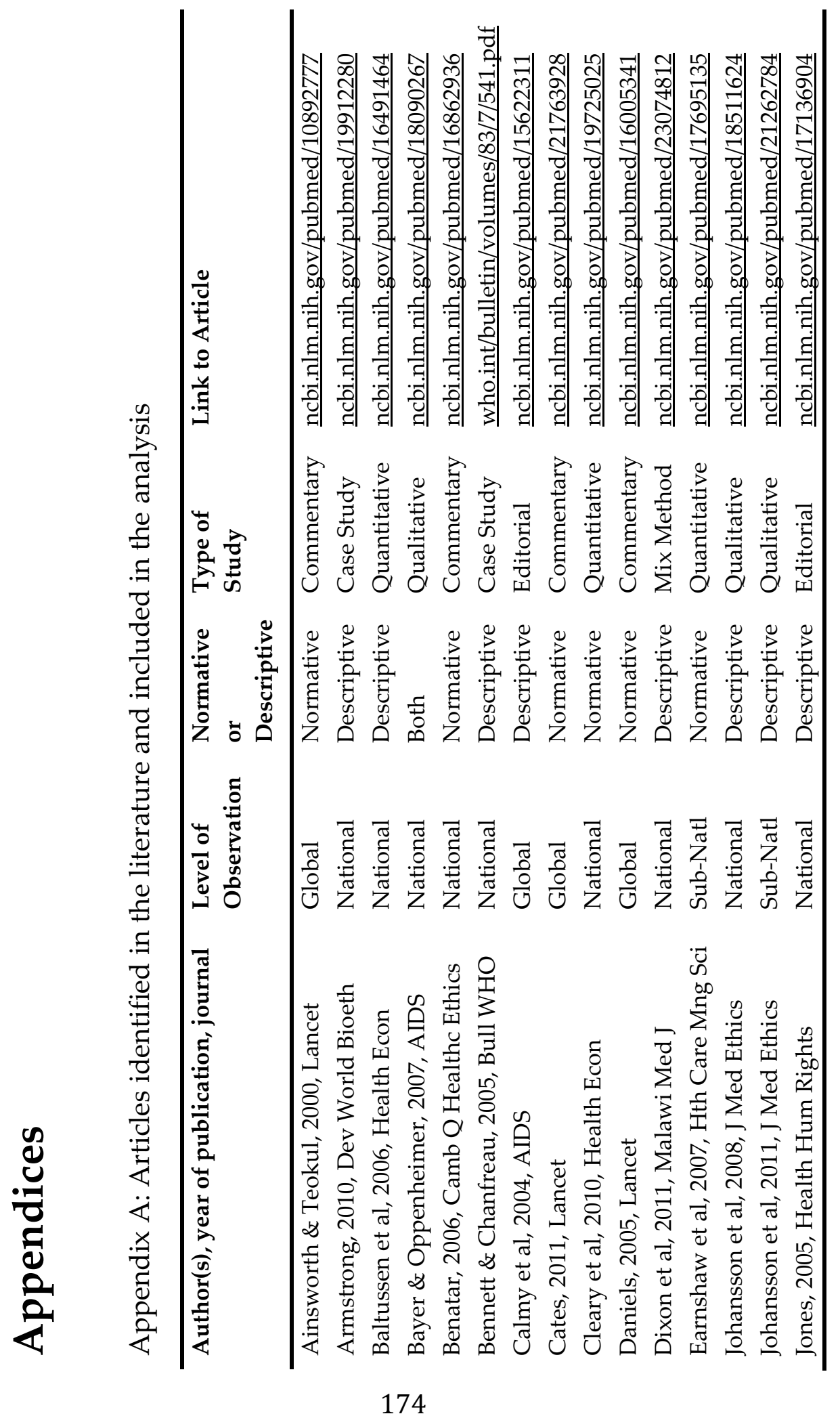




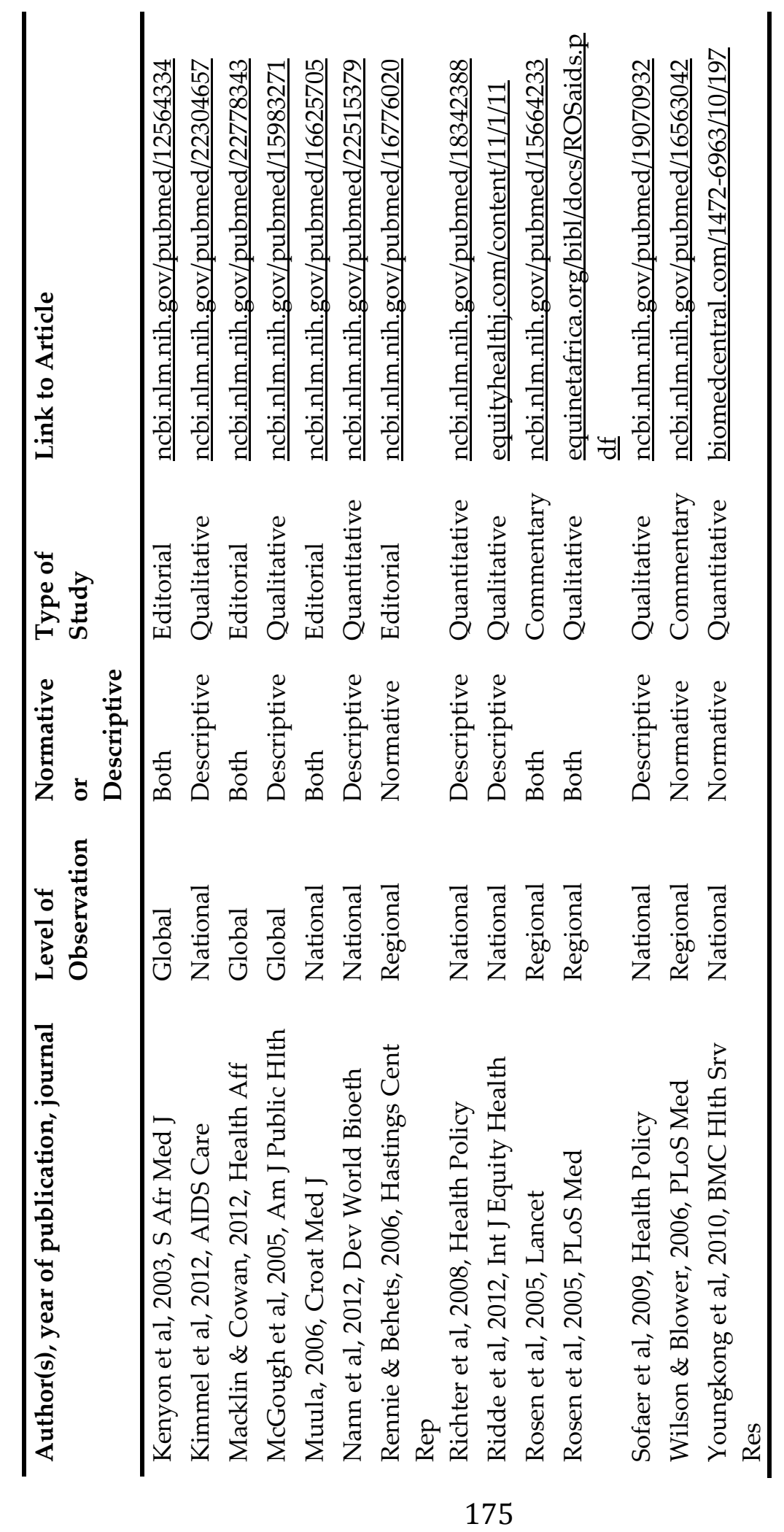


Appendix B: Comparison in Ranking after Stratification by Administrative Level of Articles in Which Criteria were Elicited

\begin{tabular}{llll}
\hline Criteria & Rank & Rank & Criteria \\
(Country Set) & (Country & $\begin{array}{l}\text { (Global } \\
\text { (Global Set) }\end{array}$ \\
& Sorted) & Sorted) & \\
\hline Equity & 1 & 1 & Severity of Disease \\
Severity of Disease & 2 & 2 & Feasibility \\
Adherence - Psych. & 3 & 2 & Equity \\
Age & 3 & 3 & Human Capital \\
Adherence - Structural & 4 & 4 & Health Outcomes \\
Fairness & 5 & 4 & Stigma and Discrimination \\
Gender & 5 & 4 & Affordability \\
Human Capital & 5 & 5 & Equal Worth of Life \\
Pregnant Women & 5 & 5 & Adherence - Structural \\
Affordability & 6 & 6 & Efficacy \\
Access Selection Committee & 7 & 6 & Fairness \\
Coverage & 7 & 6 & Pregnant Women \\
Feasibility & 7 & 6 & High-risk Populations \\
Sustainability & 7 & 6 & Sustainability \\
Accessibility & 8 & 7 & Adherence - Psych. \\
Cost-Effectiveness & 9 & 7 & Age \\
Equal Worth of Life & 9 & 7 & Gender \\
Mothers & 9 & 7 & Coverage \\
Parents & 9 & 7 & Accessibility \\
High-risk Populations & 9 & 7 & Cost-Effectiveness \\
Childbearing Age Women & 9 & 8 & Mothers \\
Stigma and Discrimination & 9 & 8 & Parents \\
Unemployed & 9 & 8 & Childbearing Age Women \\
Efficacy & 10 & 8 & Unemployed \\
Innocent Victim & 10 & 8 & Innocent Victim \\
Health Outcomes & 11 & 9 & Access Selection Committee \\
\hline & & &
\end{tabular}




\section{Appendix C: Comparison in Ranking after Stratification by Descriptive and Normative Character of Articles in Which Criteria were Elicited}

\begin{tabular}{|c|c|c|c|}
\hline $\begin{array}{l}\text { Criteria } \\
\text { (Descriptive Set) }\end{array}$ & $\begin{array}{l}\text { Rank } \\
\text { (Desc. } \\
\text { Sorted) }\end{array}$ & $\begin{array}{l}\text { Rank } \\
\text { (Norm. } \\
\text { Sorted) }\end{array}$ & $\begin{array}{l}\text { Criteria } \\
\text { (Normative Set) }\end{array}$ \\
\hline Severity of Disease & 1 & 1 & Severity of Disease \\
\hline Equity & 2 & 1 & Equity \\
\hline Adherence - Psychological & 3 & 2 & Feasibility \\
\hline Age & 4 & 3 & Human Capital \\
\hline Adherence - Structural & 5 & 3 & Stigma \& Discrimination \\
\hline Human Capital & 6 & 3 & Efficacy \\
\hline Fairness ${ }^{*}$ & 6 & 3 & Health Outcomes \\
\hline Pregnant Women & 7 & 4 & Gender \\
\hline Affordability & 7 & 4 & Pregnant Women \\
\hline Gender & 8 & 4 & Accessibility \\
\hline Coverage & 8 & 4 & Sustainability \\
\hline Feasibility & 9 & 4 & Equal Worth of Life \\
\hline Stigma \& Discrimination & 9 & 4 & High-risk Populations \\
\hline Accessibility & 9 & 4 & Age \\
\hline Sustainability & 9 & 4 & Cost-Effectiveness \\
\hline Access Selection Committee & 9 & 5 & Childbearing Age Women \\
\hline Innocent Victim & 10 & 5 & Coverage \\
\hline Mothers & 10 & 5 & Affordability \\
\hline Parents & 10 & 5 & Fairness \\
\hline Efficacy & 11 & 5 & Adherence - Structural \\
\hline Equal Worth of Life & 11 & 5 & Adherence - Psychological \\
\hline High-risk Populations & 11 & 6 & Unemployed \\
\hline Childbearing Age Women & 11 & 6 & Innocent Victim \\
\hline Unemployed & 11 & 6 & Mothers \\
\hline Health Outcomes & 12 & 6 & Parents \\
\hline Cost-Effectiveness & 12 & 6 & Access Selection Committee \\
\hline
\end{tabular}




\section{Appendix D: Comparison in Ranking after Stratification by Income Level of Country in Articles in Which Criteria were Elicited}

\begin{tabular}{|c|c|c|c|}
\hline $\begin{array}{l}\text { Criteria } \\
\text { (Upper Income Set) }\end{array}$ & $\begin{array}{l}\text { Rank } \\
\text { (Upper } \\
\text { Income } \\
\text { Sorted) }\end{array}$ & $\begin{array}{l}\text { Rank } \\
\text { (Lower } \\
\text { Income } \\
\text { Sorted) }\end{array}$ & $\begin{array}{l}\text { Criteria } \\
\text { (Lower Income Set) }\end{array}$ \\
\hline Equity & 1 & 1 & Equity \\
\hline Severity of Disease & 1 & 2 & Adherence - Psychological \\
\hline Affordability & 2 & 3 & Adherence - Structural \\
\hline Age & 3 & 4 & Human Capital \\
\hline Cost-Effectiveness & 3 & 4 & Gender \\
\hline Coverage & 3 & 4 & Severity of Disease \\
\hline Fairness & 4 & 4 & Fairness \\
\hline Feasibility & 4 & 4 & Age \\
\hline Access Selection Committee & 5 & 5 & Sustainability \\
\hline Accessibility & 5 & 5 & Pregnant Women \\
\hline Adherence - Psychological & 5 & 6 & Parents \\
\hline Adherence - Structural & 5 & 6 & Stigma and Discrimination \\
\hline Equal Worth of Life & 5 & 6 & Unemployed \\
\hline Efficacy & 5 & 6 & Feasibility \\
\hline Gender & 5 & 7 & Coverage \\
\hline Pregnant Women & 5 & 7 & Mothers \\
\hline High-risk Populations & 5 & 7 & Childbearing Age Women \\
\hline Health Outcomes & 6 & 7 & Access Selection Committee \\
\hline Human Capital & 6 & 7 & Accessibility \\
\hline Innocent Victim & 6 & 7 & Equal Worth of Life \\
\hline Mothers & 6 & 8 & Affordability \\
\hline Parents & 6 & 8 & Innocent Victim \\
\hline Childbearing Age Women & 6 & 8 & Efficacy \\
\hline Stigma and Discrimination & 6 & 8 & High-risk Populations \\
\hline Sustainability & 6 & 9 & Cost-Effectiveness \\
\hline Unemployed & 6 & 9 & Health Outcomes \\
\hline
\end{tabular}


Appendix E: Comparison in Ranking after Stratification by Epidemic Type of Focal Country in Articles in Which Criteria were Elicited

\begin{tabular}{|c|c|c|c|}
\hline $\begin{array}{l}\text { Criteria } \\
\text { (Concentrated Epidemic Set) }\end{array}$ & $\begin{array}{l}\text { Rank } \\
\text { (Conc. } \\
\text { Epi. } \\
\text { Sorted) }\end{array}$ & $\begin{array}{l}\text { Rank } \\
\text { (Gen. } \\
\text { Epi. } \\
\text { Sorted) }\end{array}$ & $\begin{array}{l}\text { Criteria } \\
\text { (Generalized Epidemic Set) }\end{array}$ \\
\hline Equity & 1 & 1 & Adherence - Psychological \\
\hline Severity of Disease & 2 & 1 & Equity \\
\hline Access Selection Committee & 3 & 2 & Fairness \\
\hline Affordability & 3 & 2 & Age \\
\hline Age & 3 & 3 & Pregnant Women \\
\hline Cost-Effectiveness & 3 & 3 & Adherence - Structural \\
\hline Efficacy & 3 & 3 & Severity of Disease \\
\hline Adherence - Psychological & 4 & 4 & Gender \\
\hline Adherence - Structural & 4 & 4 & Human Capital \\
\hline Coverage & 4 & 5 & Coverage \\
\hline Feasibility & 4 & 5 & Feasibility \\
\hline Gender & 4 & 5 & Affordability \\
\hline Human Capital & 4 & 6 & Accessibility \\
\hline Parents & 4 & 6 & Equal Worth of Life \\
\hline High-risk Populations & 4 & 6 & Stigma and Discrimination \\
\hline Sustainability & 4 & 6 & Unemployed \\
\hline Accessibility & 5 & 6 & Sustainability \\
\hline Equal Worth of Life & 5 & 7 & $\begin{array}{l}\text { Access Selection } \\
\text { Committee }\end{array}$ \\
\hline Fairness & 5 & 7 & Innocent Victim \\
\hline Health Outcomes & 5 & 7 & Mothers \\
\hline Innocent Victim & 5 & 7 & Childbearing Age Women \\
\hline Mothers & 5 & 7 & Parents \\
\hline Pregnant Women & 5 & 8 & Cost-Effectiveness \\
\hline Childbearing Age Women & 5 & 8 & High-risk Populations \\
\hline Stigma and Discrimination & 5 & 9 & Efficacy \\
\hline Unemployed & 5 & 9 & Health Outcomes \\
\hline
\end{tabular}


Appendix F: Comparison in Ranking after Stratification by Date of Article in Which Criteria were Elicited

\begin{tabular}{|c|c|c|c|}
\hline $\begin{array}{l}\text { Criteria } \\
\text { (Pre-2007 Set) }\end{array}$ & $\begin{array}{l}\text { Rank } \\
(<2007 \\
\text { Sorted })\end{array}$ & $\begin{array}{l}\text { Rank } \\
(>2007 \\
\text { Sorted })\end{array}$ & $\begin{array}{l}\text { Criteria } \\
\text { (Post-2007 Set) }\end{array}$ \\
\hline Equity & 1 & 1 & Equity \\
\hline Severity of Disease & 1 & 2 & Severity of Disease \\
\hline Feasibility & 2 & 3 & Adherence - Psychological \\
\hline Human Capital & 3 & 4 & Adherence - Structural \\
\hline Adherence - Structural & 4 & 4 & Age \\
\hline Affordability & 4 & 5 & Human Capital \\
\hline Age & 4 & 5 & Affordability \\
\hline Pregnant Women & 4 & 5 & Fairness \\
\hline Sustainability & 4 & 6 & Pregnant Women \\
\hline Adherence - Psychological & 5 & 6 & Gender \\
\hline Equal Worth of Life & 5 & 7 & Feasibility \\
\hline Fairness & 5 & 7 & Access Selection Committee \\
\hline Gender & 5 & 7 & Stigma \& Discrimination \\
\hline Stigma \& Discrimination & 5 & 7 & High-risk Populations \\
\hline Accessibility & 6 & 7 & Coverage \\
\hline Coverage & 6 & 8 & Sustainability \\
\hline Health Outcomes & 6 & 8 & Equal Worth of Life \\
\hline Cost-Effectiveness & 7 & 8 & Accessibility \\
\hline Efficacy & 7 & 8 & Unemployed \\
\hline Mothers & 7 & 8 & Cost-Effectiveness \\
\hline Parents & 7 & 8 & Efficacy \\
\hline High-risk Populations & 7 & 9 & Health Outcomes \\
\hline Childbearing Age Women & 7 & 9 & Mothers \\
\hline Access Selection Committee & 8 & 9 & Parents \\
\hline Innocent Victim & 8 & 9 & Childbearing Age Women \\
\hline Unemployed & 8 & 9 & Innocent Victim \\
\hline
\end{tabular}




\begin{tabular}{|c|c|}
\hline & $\begin{array}{l}\text { Resource needs/costs/duration } \\
\text { Availability of data/ability to recruit } \\
\text { Ethical/legal/social issues } \\
\text { Probe: Use different key populations (MSM, FSW, IDU), and } \\
\text { different areas (urban cities, rural provinces) to probe specific } \\
\text { issues that they expect to encounter with using the method for } \\
\text { estimating the size of that key population. } \\
\text { Probe: Set some conflicting scenarios to understand importance of } \\
\text { criteria to the interviewee. For example one method is costly and } \\
\text { produces valid and precise results. Another is less costly but also } \\
\text { less precise. Which do you choose? }\end{array}$ \\
\hline $\begin{array}{l}\text { Current } \\
\text { Size } \\
\text { Estimate }\end{array}$ & $\begin{array}{l}\text { Q: Before this current size estimation was started, what were } \\
\text { some of your expectations? } \\
\text { Probe: How do you think the survey would go? What did you } \\
\text { think the community response would be? What did you think the } \\
\text { results would be? How do you feel about the method now? }\end{array}$ \\
\hline \multicolumn{2}{|c|}{$\begin{array}{l}\text { Closing } \\
\text { Is there anything else you think is important in selecting size estimation } \\
\text { methods that you think we should talk about? }\end{array}$} \\
\hline \multicolumn{2}{|c|}{ Summarize } \\
\hline \multicolumn{2}{|c|}{ Thank participant } \\
\hline Provide & ps to particip \\
\hline
\end{tabular}


Appendix H: Summary of Methods of Estimating Population Size

\begin{tabular}{|c|c|c|c|}
\hline Method & Description & Strength & Weakness \\
\hline Census & $\begin{array}{l}\text { Count all } \\
\text { members of } \\
\text { population }\end{array}$ & $\begin{array}{l}\text { Simple to } \\
\text { understand }\end{array}$ & $\begin{array}{l}\text { Time consuming; } \\
\text { costly; validity } \\
\text { questionable for } \\
\text { mobile or hidden } \\
\text { populations; }\end{array}$ \\
\hline Enumeration & $\begin{array}{l}\text { Develop } \\
\text { Sampling } \\
\text { Frame, then } \\
\text { count all in } \\
\text { frame }\end{array}$ & $\begin{array}{l}\text { Less costly } \\
\text { than census; }\end{array}$ & $\begin{array}{l}\text { Requires reliable } \\
\text { sampling frame } \\
\text { (statistical expertise } \\
\text { needed); validity } \\
\text { questionable for } \\
\text { mobile or hidden } \\
\text { populations; }\end{array}$ \\
\hline Capture-Recapture & $\begin{array}{l}\text { Calculate total } \\
\text { based on two } \\
\text { independent } \\
\text { samples }\end{array}$ & $\begin{array}{l}\text { Easy to use; no } \\
\text { statistical } \\
\text { expertise } \\
\text { required; }\end{array}$ & $\begin{array}{l}\text { Difficult to meet } \\
\text { assumption in the field } \\
\text { (independent, } \\
\text { uncorrelated samples, } \\
\text { equal chance of } \\
\text { selection, } \\
\text { identification of } \\
\text { captures, no } \\
\text { migration, large } \\
\text { sample size) }\end{array}$ \\
\hline Multiplier & $\begin{array}{l}\text { Calculate size } \\
\text { based on a } \\
\text { sub- } \\
\text { population } \\
\text { census and a } \\
\text { sampled } \\
\text { proportion of } \\
\text { total }\end{array}$ & $\begin{array}{l}\text { Easy to use; no } \\
\text { statistical } \\
\text { expertise } \\
\text { required; } \\
\text { Flexible } \\
\text { method; }\end{array}$ & $\begin{array}{l}\text { Independent data } \\
\text { sources required; } \\
\text { alignment of age, } \\
\text { geography, and time } \\
\text { periods between two } \\
\text { sources is difficult; } \\
\text { data from existing } \\
\text { sources may be } \\
\text { inaccurate; }\end{array}$ \\
\hline
\end{tabular}




\begin{tabular}{|c|c|c|c|}
\hline Population Survey & $\begin{array}{l}\text { Direct survey } \\
\text { of general } \\
\text { population } \\
\text { about } \\
\text { membership } \\
\text { in group being } \\
\text { counted }\end{array}$ & $\begin{array}{l}\text { Surveys are } \\
\text { common, easy } \\
\text { to analyze, } \\
\text { simple to } \\
\text { understand } \\
\text { and explain; }\end{array}$ & $\begin{array}{l}\text { Difficult to use for } \\
\text { stigmatized } \\
\text { populations/behaviors; } \\
\text { limited to households, } \\
\text { schools or institutions } \\
\text { in sampling frame; } \\
\text { perceptions of } \\
\text { confidentiality can } \\
\text { distort responses; }\end{array}$ \\
\hline $\begin{array}{l}\text { Network } \\
\text { Scale-up }\end{array}$ & $\begin{array}{l}\text { Survey of } \\
\text { general } \\
\text { population } \\
\text { about size of } \\
\text { their network } \\
\text { who belong to } \\
\text { group being } \\
\text { counted }\end{array}$ & $\begin{array}{l}\text { Estimates } \\
\text { drawn from } \\
\text { general } \\
\text { population; } \\
\text { may produce } \\
\text { more valid } \\
\text { responses than } \\
\text { estimates } \\
\text { drawn from } \\
\text { key } \\
\text { populations } \\
\text { (due to } \\
\text { reduced } \\
\text { respondent } \\
\text { bias); one } \\
\text { survey can } \\
\text { produce } \\
\text { multiple pop } \\
\text { estimates; }\end{array}$ & $\begin{array}{l}\text { Difficult to estimate } \\
\text { average network size; } \\
\text { may not represent sub- } \\
\text { groups/behaviors } \\
\text { hidden from or } \\
\text { stigmatized by general } \\
\text { population; }\end{array}$ \\
\hline
\end{tabular}

Source: Adapted from "Guidelines on Estimating the Size of Populations Most at Risk to HIV". WHO and UNAIDS. 2010. 
Appendix I: The discrete choice experiment choice scenarios in the survey instrument

\section{Survey on Priority Criteria in the Viet Nam HIV Response}

Welcome to the survey on Criteria for HIV Program Selection in Viet Nam. This survey is anonymous and is carried out for academic purposes only. You may exit and clear the survey at any time while answering the questions, should you feel uncomfortable.

If you agree to start, please press the "NEXT" button.

\section{Next}




\section{Survey on Priority Criteria in the Viet Nam HIV Response}

Please tell us a bit about yourself:

Your Gender:

$\square$ Male $\quad \square$ Female

Your Age:

$\square<22 \quad \square 22-25 \quad \square 26-30 \quad \square 31-40 \quad \square 41-50 \quad \square 51-60 \quad \square>60$

Your Country of Origin:

$\square$ Việt Nam $\quad \square$ Other:

Your organization:

$\square$ Government

$\square$ United Nations

$\square$ Donor Agency

$\square$ Research/Academic Institution

$\square$ Civil Society/Community Organization

$\square$ Other:

Your area of work (you can choose multiple options):

$\square$ Programs

$\square$ Policy

$\square$ Management

$\square$ Monitoring \& Evaluation

$\square$ Other:

Years of experience working in HIV response:

Have you been involved in making decisions on HIV programs?

$\square$ Yes $\quad \square$ No

Have you been responsible for making decisions about HIV programs?

$\square$ Yes $\quad \square$ No 


\section{Survey on Priority Criteria in the Viet Nam HIV Response}

Imagine a situation when you have to decide on a suitable program for HIV response in Viet Nam, given a fixed budget. You could base your decision on several criteria:

Effectiveness: Years of life saved from death or disease Sustainability: Maintaining or reducing resource needs Prevention/Treatment Spending Ratio: Spending on prevention versus spending on treatment

Cost-Effectiveness: Economic returns of program versus cost of program Feasibility: Likelihood of achieving the expected scale and rate of increase in coverage set by the program

Please order these criteria in terms of their importance to you if you had to make such a decision:

Your most important criterion should be on the top right, moving through to your least important criterion.

Your choices Your ranking

Effectiveness

Sustainability

Prevention/Treatment Spending Ratio

Cost-Effectiveness

Feasibility

\section{Next}




\section{Survey on Priority Criteria in the Viet Nam HIV Response}

In the following pages you will be presented with seven scenarios. In each scenario you will be asked to choose between two programs for HIV response in Viet Nam. All programs carry the same cost of $\$ 80$ Million USD. The two programs presented to you in each scenario are similar in every way, except for those attributes that are highlighted.

The purpose of this survey is not to find the best programs. Therefore, there is no wrong answer to the choices you make. Please use your values to choose between the programs presented in each scenario.

\section{Next}




\section{Survey on Priority Criteria in the Viet Nam HIV Response}

Imagine a situation when you have to decide on a suitable program for HIV response in Viet Nam.

Which of these programs would you choose? Both programs have the same cost of $\$ 80$ Million USD. To make it easier for you, we have highlighted the characteristics that differ between the two programs.

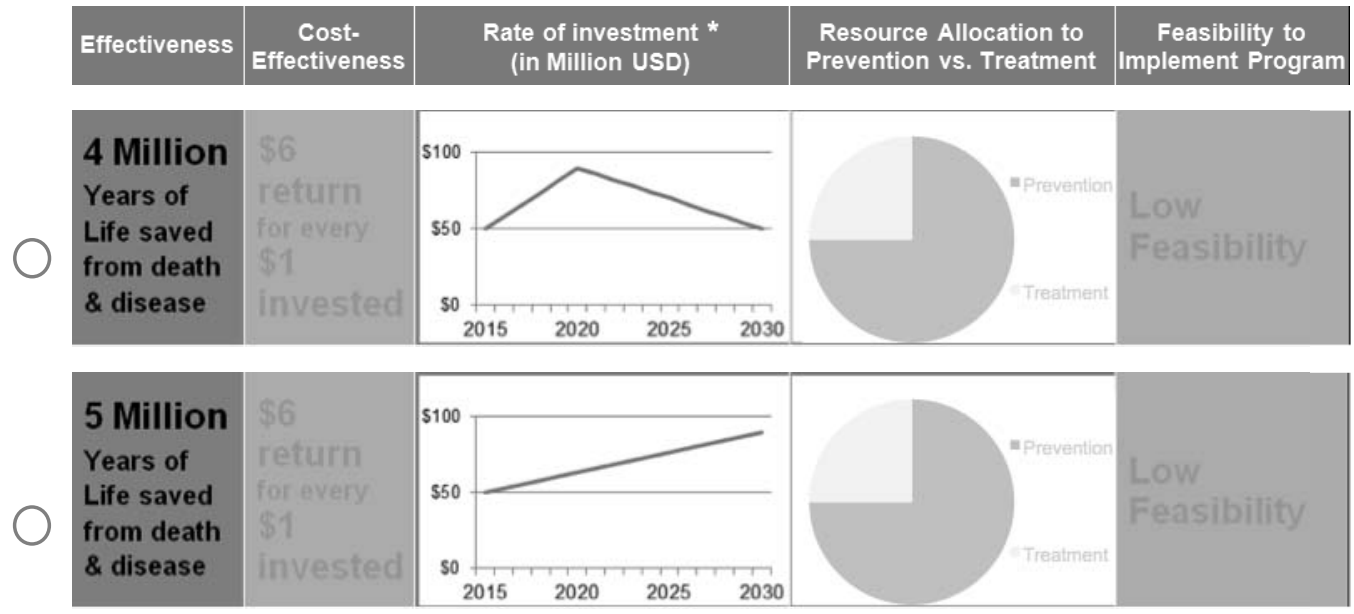

\section{Next}




\section{Survey on Priority Criteria in the Viet Nam HIV Response}

Imagine a situation when you have to decide on a suitable program for HIV response in Viet Nam.

Which of these programs would you choose? Both programs have the same cost of $\$ 80$ Million USD. To make it easier for you, we have highlighted the characteristics that differ between the two programs.

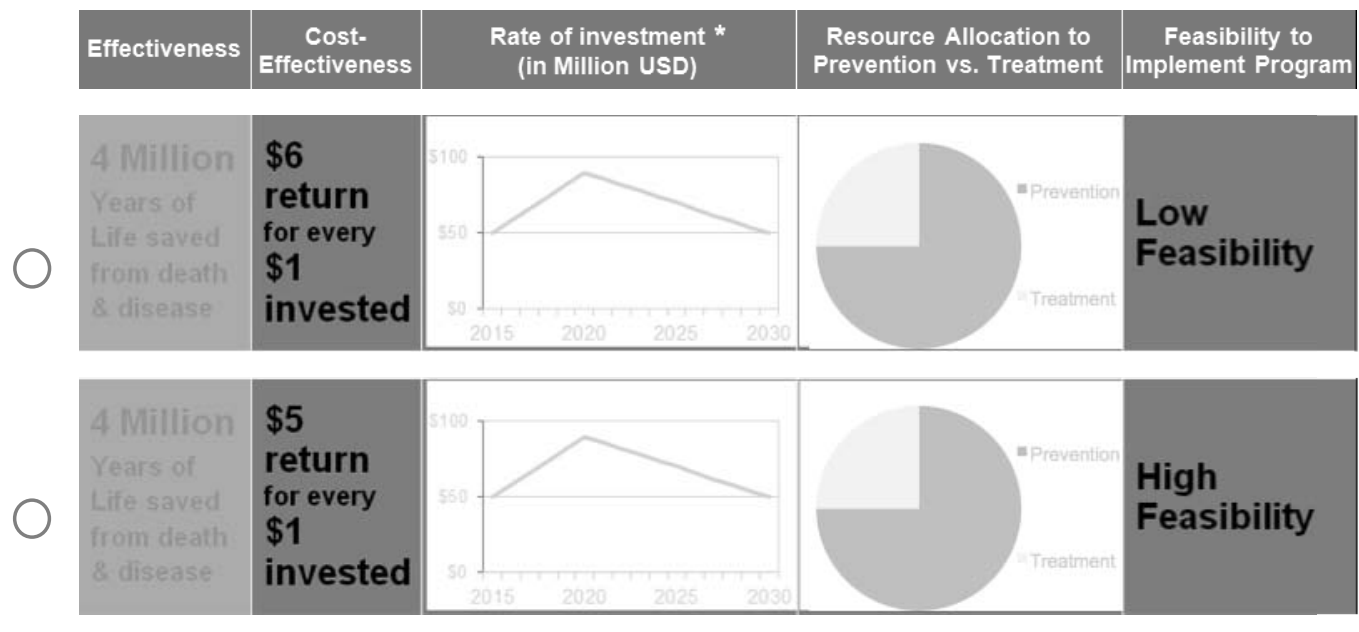

\section{Next}




\section{Survey on Priority Criteria in the Viet Nam HIV Response}

Imagine a situation when you have to decide on a suitable program for HIV response in Viet Nam.

Which of these programs would you choose? Both programs have the same cost of $\$ 80$ Million USD. To make it easier for you, we have highlighted the characteristics that differ between the two programs.

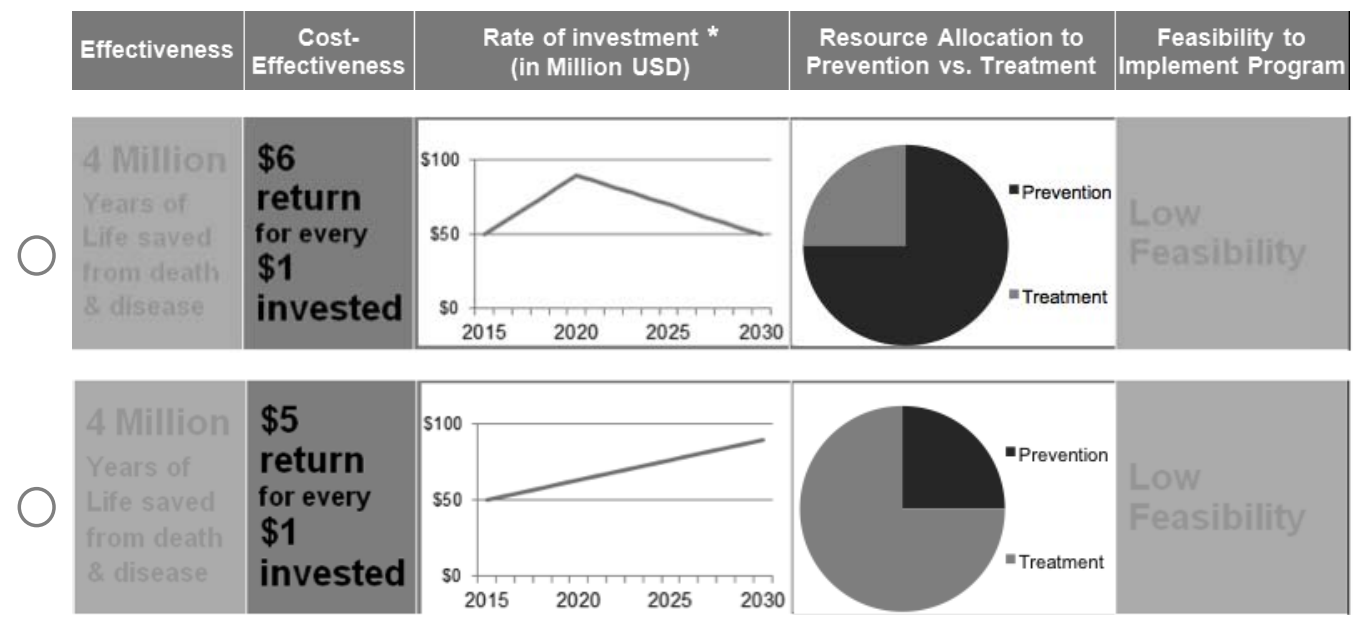

\section{Next}




\section{Survey on Priority Criteria in the Viet Nam HIV Response}

Imagine a situation when you have to decide on a suitable program for HIV response in Viet Nam.

Which of these programs would you choose? Both programs have the same cost of $\$ 80$ Million USD. To make it easier for you, we have highlighted the characteristics that differ between the two programs.

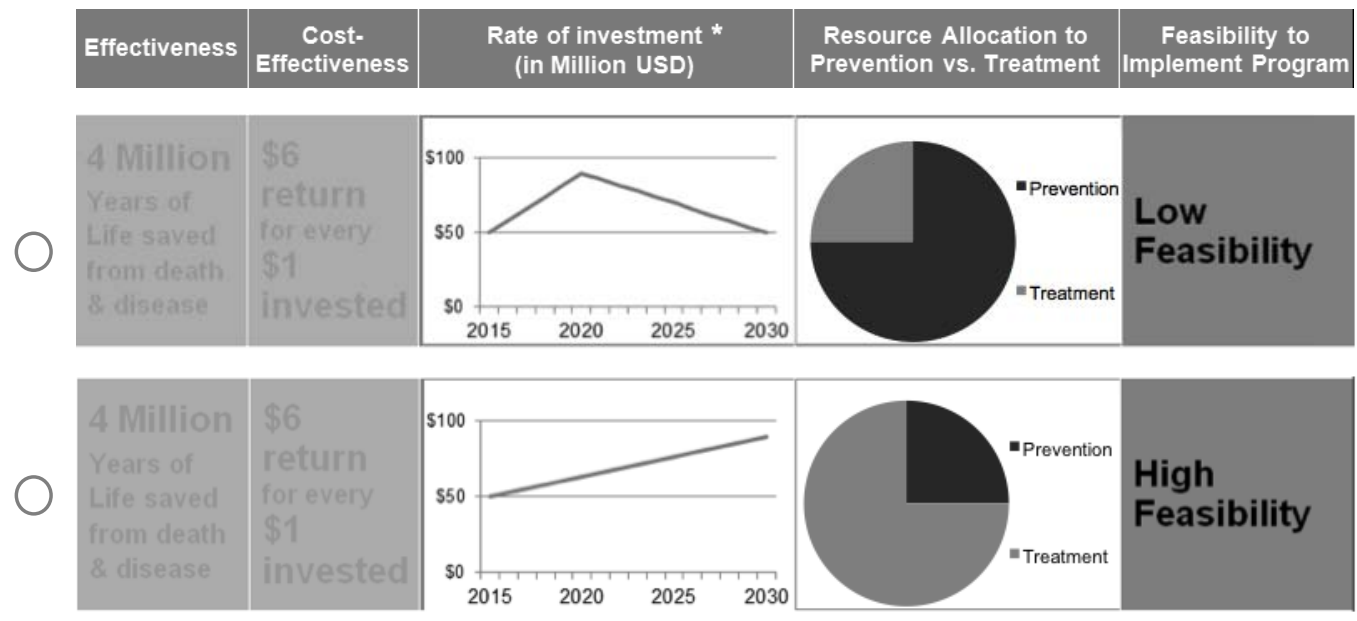

\section{Next}




\section{Survey on Priority Criteria in the Viet Nam HIV Response}

Imagine a situation when you have to decide on a suitable program for HIV response in Viet Nam.

Which of these programs would you choose? Both programs have the same cost of \$80 Million USD. To make it easier for you, we have highlighted the characteristics that differ between the two programs.

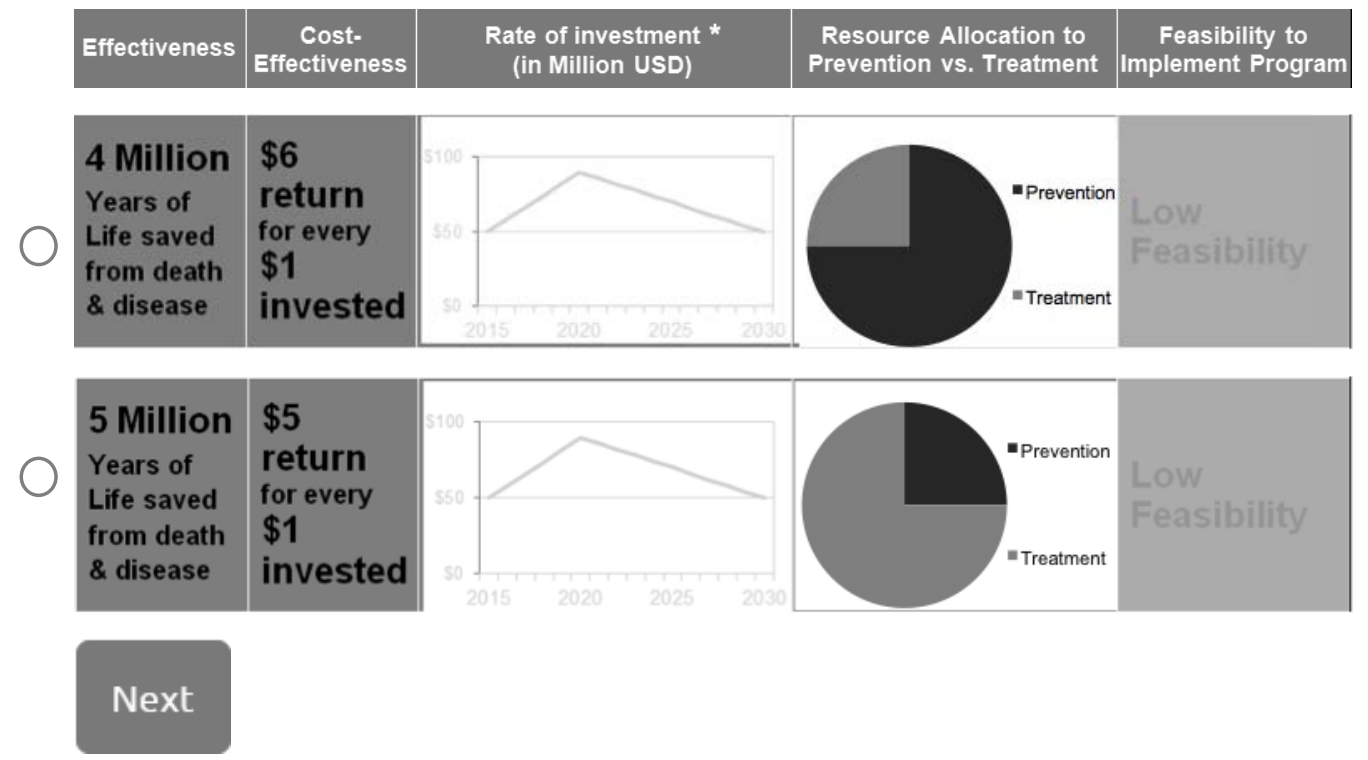




\section{Survey on Priority Criteria in the Viet Nam HIV Response}

Imagine a situation when you have to decide on a suitable program for HIV response in Viet Nam.

Which of these programs would you choose? Both programs have the same cost of $\$ 80$ Million USD. To make it easier for you, we have highlighted the characteristics that differ between the two programs.

\begin{tabular}{|c|c|c|c|c|} 
Effectiveness & $\begin{array}{c}\text { Cost- } \\
\text { Effectiveness }\end{array}$ & $\begin{array}{c}\text { Rate of investment * } \\
\text { (in Million USD) }\end{array}$ & $\begin{array}{c}\text { Resource Allocation to } \\
\text { Prevention vs. Treatment }\end{array}$ & $\begin{array}{c}\text { Feasibility to } \\
\text { Implement Program }\end{array}$ \\
\hline
\end{tabular}
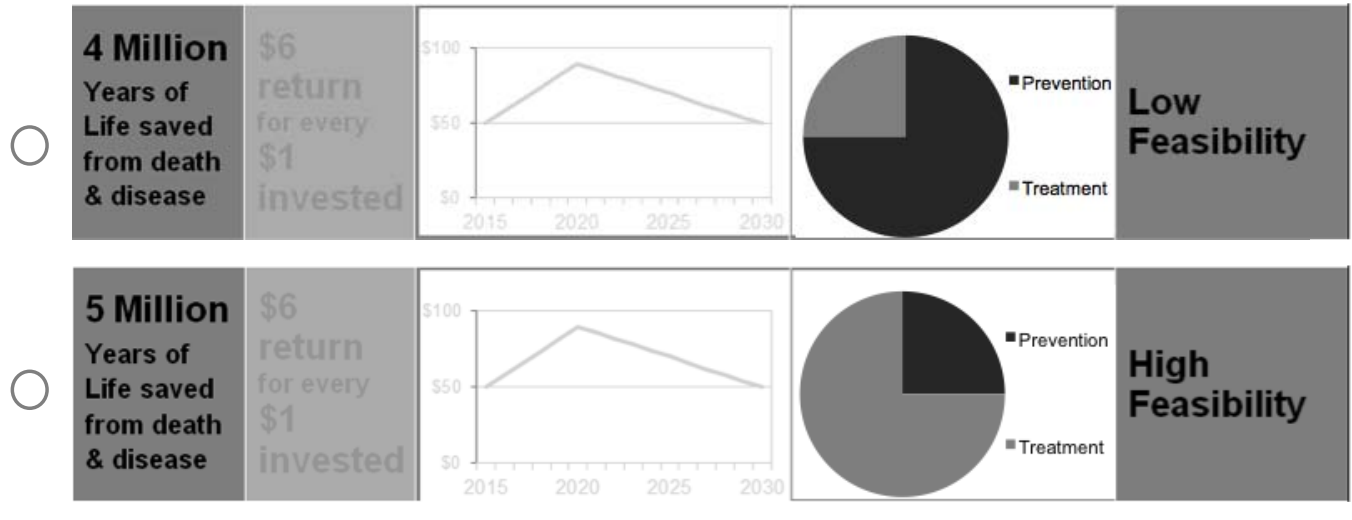

\section{Next}




\section{Survey on Priority Criteria in the Viet Nam HIV Response}

Imagine a situation when you have to decide on a suitable program for HIV response in Viet Nam.

Which of these programs would you choose? Both programs have the same cost of \$80 Million USD. To make it easier for you, we have highlighted the characteristics that differ between the two programs.

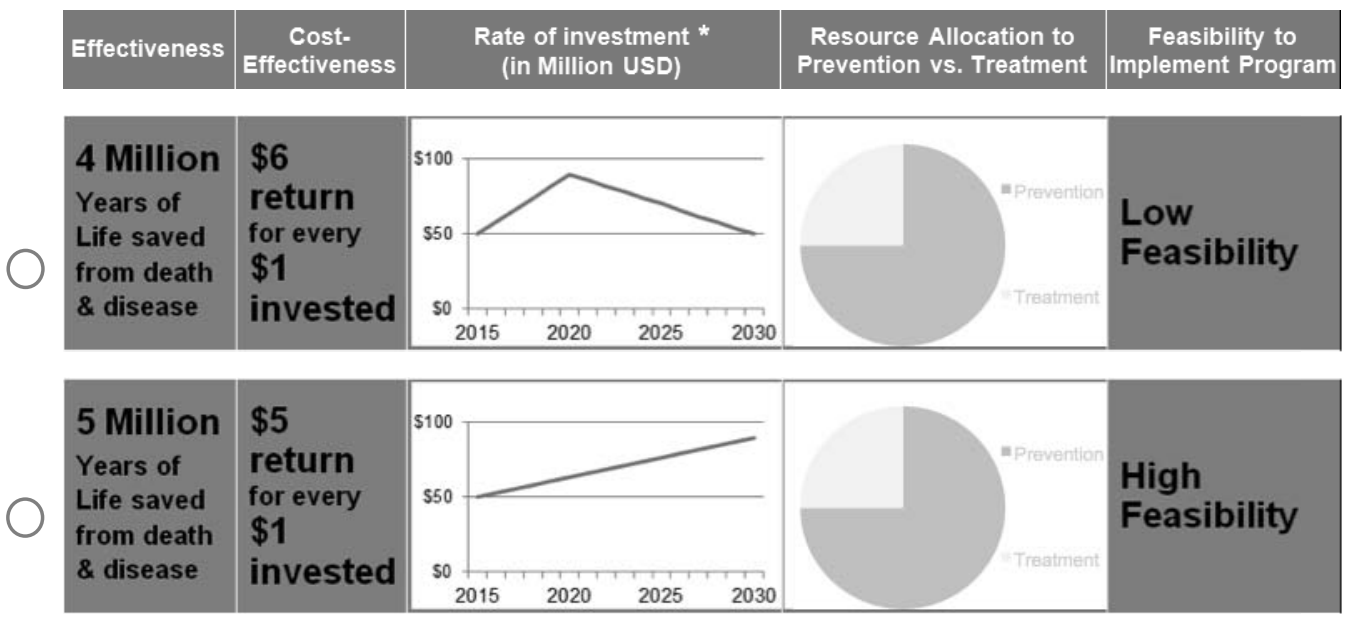

\section{Next}




\section{Summary}

This research focuses on the prioritization of the HIV response. Today there are over 37 Million people who are living with HIV although fewer than half of them are able to access lifesaving treatment. And while the Sustainable Development Goals call for ambitious targets to urgently control the epidemic and ends AIDS as a public health threat by 2030, global financing for the HIV response has stagnated in recent years and donor AIDS funding has started to recede. With the political and financial will to end AIDS waning, and given the constrained resources and ambitious targets, prioritization is urgently needed.

This dissertation considers how to prioritize the HIV response using the structure of the multi-criteria analytical framework. This framework is a branch of operations research, which has been shown to work in low- and middleincome countries to prioritize a range of development issues. It facilitates decision-making transparently and consistently, while considering multiple criteria, although experts caution that more experience is needed in its application. Two particular problems that commonly afflict decision-making in the health sector and require attention are (1) lack of quality information on programs and policy choices and the consequences of the choices, and (2) a neglect of stakeholder involvement in decision-making.

The principle aim of this research is then to study the appropriateness and applicability of the multi-criteria analytical framework for prioritizing the HIV response in a multi-stakeholder decision-making process. The chapters of this dissertation that respond to the stated aim are summarized here as follows. 
Chapter 1 provides a timeline of the global HIV epidemic and response, including a history of international political and financial commitments made toward ending the AIDS epidemic as a public health threat. It then introduces the motivation for prioritization of the HIV response at this moment in the history of HIV and AIDS. The unsolved priority setting challenges are outlined and the theoretical origins and debates in the priority setting field are reviewed. The aim of this research and four related research questions are articulated in Chapter 1 . The chapter then concludes with a brief outline of the dissertation.

In line with the first stages of the multi-criteria decision analysis framework, Chapter 2 of this dissertation explores and articulates the criteria to prioritize the HIV response. The principle question being sought to answer in this Chapter is: What are the relevant criteria to prioritize the programs, policies, investments, workforce and technologies that are utilized in responding to the HIV epidemic? To respond to this question, a systematic review of literature was undertaken to identify the existing criteria relevant to prioritizing the HIV response. The review included literature since the year 2000 to present, which dealt with the breadth of criteria involved in decision-making and which included operational definitions of criteria.

Cluster analysis was used to classify and structure the criteria identified in the systematic review into thematic groupings. The articles from which criteria were drawn were stratified by the income group of countries under study, the administrative level, type of the epidemic, whether the article was characterized as normative or descriptive, and the year of publication of the article. These stratifications were used to explore the similarities and differences between 
criteria, and compare their frequency of occurrence. Identified HIV and AIDS criteria were also compared with the criteria from the healthcare sector.

From the articles that met the inclusion criteria of the systematic review, thirty unique criteria were identified. These criteria were subsequently clustered into 18 thematic groups. Equity and severity of disease were the most frequently occurring criteria. A theme that developed around the criteria during cluster analysis was on "social justice", emphasizing the ethical imperative of prioritizing the most marginalized populations despite higher costs per lifeyear saved.

What is concerning however, is that one of the widest gap between the healthcare set of criteria and HIV set of criteria is around criteria of vulnerable populations, illustrating the gap in priorities between the sectors, and underlining the need to consider multi-sectorial criteria in any possible integration of HIV programs into the health systems. Moreover, we see populations at high risk of HIV infection rank in the lower half of all criteria regardless of stratification, even between concentrated and generalized epidemics, despite these populations carrying a greater burden of the epidemic, and most at risk of being infected. In line with the findings of Chapter 2, and recognizing the priorities that contributed to the success of the HIV response thus far, we make a recommendation that the discourse around prevention among high-risk and vulnerable groups be amplified in consideration of policies of prioritization, particularly in concentrated epidemics.

In stratification of criteria, it was observed that the feasibility criterion appeared frequently in the normative set, but infrequently in the descriptive set, 
suggesting that this criterion is often recommended but seldom used. Popularity of feasibility also declined over time, appearing less frequently after 2007. We hypothesize this may be due to weak information systems in earlier years, which may make the use of criteria without operational definitions or metrics more favourable in reason-based, political decision-making.

A challenge that emerged in Chapter 2 is how to operationalize and measure the identified criteria and themes in order to inform a multi-criteria priority setting process. In line with this question, and related to the multi-criteria decision analysis framework's performance measurement stage, Chapter 3 is concerned with evidence availability and use in HIV response planning. The principle question being investigated in Chapter 3 is: To what extent is reliable and good quality evidence available, accessible and used in planning of the HIV response?

To answer this question, we developed an instrument with several dimensions to measure the quality and coverage of evidence used in national HIV plans. Using standard content analysis, we reviewed 27 NSP from 21 countries. These countries are home to two out of every three people living with HIV globally. To ensure the accuracy of measurements, we analysed the instrument's reliability, internal consistency, and external validity.

Our analysis of the use of evidence in national HIV planning demonstrates a trend in increasing evidence use over the years. This finding is consistent with studies that suggest increasing investments on monitoring and evaluation are paying off in terms of improved generation of evidence. Moreover, the types of evidence used in national HIV plans are more diverse and the evidence quality 
is greater today than it was in the past. Data on epidemic drivers were the most frequently used type of evidence in national plans.

Following the lead from Chapter 2, we also noted in our investigation of evidence in the national HIV plans that although overall there is adequate evidence related to key populations at higher risk of HIV, there is great variance across the plans in quality and coverage of this evidence. In particular, we noted only 3 of the 27 national plans provide any evidence on men who have sex with men, and only one provided a population size for this key population. Expenditure data and impact/outcome evaluation were also infrequently used in planning. Based on these findings, we made a number of recommendations, including better alignment of timing of program reviews with the national HIV planning processes for use of generated evidence in the decision-making, and incentivizing continued use of evidence by including measures of evidencebased planning in international benchmarks of governance.

Chapter 4 brings attention to a problem that often afflicts policy and planning in the health sector, which is lack of involvement of stakeholders in the decision-making. The question being asked in Chapter 4 is: What difference does the inclusion of diverse stakeholders make in decision-making processes of the HIV response? This question is approached from the perspective of the themes that emerged in Chapters 2 and 3, in terms of weak evidence on key atrisk populations, and infrequent reference to these populations in low- and middle-income countries faced with a concentrated epidemic. Therefore a specific focus is taken on differences in perspectives of multiple stakeholders on a key evidence generation activity, surveillance of the size of key at-risk populations in a country with a concentrated epidemic, namely Viet Nam. 
To explore the perspectives of different stakeholders, we conducted 16 in-depth interviews with health program managers, technical experts in surveillance, and members of the community of people at risk of HIV in one urban city and one rural province of Viet Nam. Transcripts of the interviews were reviewed for significant statements pertaining to criteria, including variations and agreement around those criteria. The emerging criteria were validated against an established framework for prioritizing evidence generation methods. Eleven themes emerged as having particular relevance to the evidence generation related to key populations at risk of HIV in Viet Nam. Findings on missing criteria, inclusive participation, community perspectives and conflicting weight and direction of criteria in this case study provide insights to help improve on an applied framework for the prioritization of evidence generation methods.

Overall our findings suggest that stakeholder involvement improves definition and coverage of relevant criteria, but it introduces conflicts in weight and direction of the criteria. To reconcile the different perspectives and conflicts, we recommend a group exercise to organize criteria into a hierarchical structure, combining redundant criteria and decomposing alternative definitions, with the additional benefit of raising consciousness about the causes of conflicts. On criteria with different weight and direction we recommend doing a weight analysis under a multi-stakeholder scenario.

Chapter 5 is about choices. The principles question being investigated in this Chapter is: How do different stakeholders rate the relative importance of criteria for prioritizing the HIV response? To answer this question, we designed a discrete choice experiment to measure the relative trade-off of criteria for 
decision-makers and stakeholder. The discrete choice experiment was set in Viet Nam, and included eight HIV program scenarios that were developed using epidemiological estimation and projection modeling software. These scenarios were characterized by the top five criteria that were elicited in previous chapters and were deemed relevant to the context of Viet Nam. The five criteria were cost-effectiveness, feasibility, sustainability, treatment-toprevention spending ratio, and effectiveness.

Based on recommendations emerging from Chapter 4, the discrete choice experiment included 69 participants with diverse representation from the government, civil society, and members of donor community or development partners. The participants were tasked with choosing between pairs of scenarios. The choices of the respondents in the experiment were analysed to understand the relative trade-off of criteria when choosing between the HIV program scenarios. The participants' revealed choices in the discrete choice experiment were also compared to their stated preferences in manually ranking the criteria.

Findings in Chapter 5 revealed that all else being equal, participants prefer a program that is most feasible, front-loaded for sustainability, has a higher proportion of investment on prevention, saves more lives and prevents more infections, and is more cost-effective, in that order. The criteria of feasibility, sustainability and treatment-to-prevention spending ratio were all given high importance by participants from civil society and government, demonstrating that there are greater similarities than differences in choices of these two groups. These similarities in rankings of criteria can create common grounds for future policy dialogues between stakeholders. Working in HIV programming or 
being involved in decision-making had a significant effect on how participants ranked the criteria. Our findings also indicate that when the relative importance of criteria are stratified by the participants' professional duties, the responses follow closely the concerns in a traditional model of public service governance. That is, those involved in decision-making are more concerned with the effectiveness of the programs they choose, and others at lower levels of the governance structure have operational concerns such as the feasibility of the programs chosen. Given this potential filtering of criteria in top-down organizations, we recommend iterative models of planning or greater involvement of stakeholders at all stages of planning to ensure relevant criteria are considered at appropriate decision points in prioritizing the HIV response.

Chapter 6 discusses the main findings of this dissertation in response to the four questions of the research: (1) What are the relevant criteria to prioritize the programs, policies, investments, workforce and technologies that are utilized in responding to the HIV epidemic? (2) To what extent is reliable and good quality evidence available, accessible and used in planning for the HIV response? (3) What difference does the inclusion of diverse stakeholders make in decisionmaking processes of the HIV response? (4) How do different stakeholders rate the relative importance of criteria for prioritizing the HIV response? Five crosscutting statements respond to these questions by bringing together the findings from this dissertation. Those statements are as follows:

Summary Statement 1 says that to be effective, the process of priority setting of the HIV response requires an iterative, multi-stakeholder approach. That proposed approach, according to the findings of this dissertation, and in line with the multi-criteria decision analysis framework is to (1) review the relevant 
criteria, (2) model program options based on quality evidence and along the identified criteria, and (3) analyze criteria weights and score alternative program options in one combined step. It is also important to build iteration into the process. Given the potential filtering of criteria in top-down organizations, and since it is not possible or probable for every stakeholder to be involved at every stage of planning, iteration can help ensure the relevant criteria of different stakeholders are given adequate consideration.

Statement 2 says that the global fall in HIV funding and the reintegration of HIV programs into the health sector have renewed the focus on feasible and sustainable programs. The premise of this statement is that donor funding for HIV has been declining in recent years. And with this decline in funding, the costly parallel programs devised to quickly mount an emergency response to the HIV epidemic are being reintegration into the health system. This reintegration in turn has diverted the conversation from cost and costeffectiveness of programs to the question of feasibility and sustainability of the programs. That is, taxpaying constituents whose tax-money makes up the bulk of official development assistance wanted to know the cost-effectiveness of the donor funded programs. Today, stakeholders and governments in low- and middle-income countries want to know if the programs they pursue are feasible and sustainable.

Statement 3 says the use of evidence in HIV planning and policy-making has been increasing over the years, particularly use of data on drivers of the epidemic. We started our research in Chapter 3 on the premise that monitoring and evaluation systems have been strengthening, so quality data should be more available. We found in Chapter 3 that indeed evidence was available and 
being used in program planning and policy setting. However, we also noted gaps in data availability and use, such as in use of operations research and impact evaluation. We posited that this particular gap was likely due to timing of availability of program reviews that made them unusable at the right time in planning. We also noted gaps and variance in evidence around key populations in planning, which brought us to statement 4 .

Statement 4 says that the gaps and variance in quality evidence on key at-risk groups take the focus away from these populations in national strategic plans and programs. We noticed in Chapter 4 that stigma, lack of technical knowledge in surveillance methods, and conflicting views of stakeholders were contributing factors to lack of key population involvement in evidence generation. This lack of involvement led to a lack of quality evidence on key population. Lack of quality had the consequent lack of trust in that evidence when and if it was used in HIV program planning. And lack of evidence use further reduced attention on key populations in programs. The implication here is that if there are no measures for a program, an intervention, or a population, then they may get neglected. Or as the UN Secretary General Ban Ki Moon puts it more simply, "if you're not counted, you don't count".

Statement 5 says that epidemic control and reduction of stigma for key at-risk groups requires their involvement in the HIV response priority setting process. This statement evolved from observations across the prioritization stages which saw that who is involved is related to what criteria are considered. And criteria considered determines the program components that are prioritized. And program prioritization aims to achieve the stated objectives of epidemic control and stigma reduction. So involvement of key populations is ultimately linked to 
the outcome of HIV programs. But we also argue in this dissertation that involvement without adequate capacity is not meaningful involvement. Often value-decisions are decentralization to technical experts as a way of exclusion of stakeholders who do not have the adequate technical capacity to participate, in what is known as technocratic decision-making. Our recommendation in this regard is for greater technical literacy of stakeholders, through capacity building. In this way, the remaining donor financing could be shifted to invest in technical assistance to low- and middle-income countries instead of programmatic assistance.

This dissertation started with giving some important reasons why prioritizing the HIV response is important and urgent, such as the ambitious targets, the reduced funds, and the gap in HIV treatment. However, a critical reason that guides prioritization as revealed in this dissertation is social justice. Prioritization is in the end the very opposite of ignoring the most marginalized populations. Prioritization is taking the vulnerable, the oppressed and the abused out of the margins and placing them at the center of the response. Prioritization is the operationalization of an equitable HIV response.

Overall, this dissertation has shown that the elements of the multi-criteria analytical framework are applicable to prioritizing the HIV response. There is readiness for the application of the framework in terms of evidence availability, defined criteria, and feasibility of involvement of multiple stakeholders in the process. As the financial and political will to end the AIDS epidemic as a public health threat wanes, findings and recommendations of this dissertation can give guidance on a fair, accountable and transparent path to prioritizing the HIV response to treat the most vulnerable and to leave no one behind. 


\section{Valorization Addendum}

This section discusses the valorization opportunities offered by the dissertation. The topic of this research related to priority setting in the HIV response is highly policy relevant. The main results and policy recommendations of this dissertation can be relevant for a range of target audiences including governments, development agencies, and civil society organizations.

Results of the research in this dissertation provide the first indication that evidence use in HIV planning and policymaking has been increasing over the years. However, stagnation and decline in donor funding for monitoring and evaluation systems, along with integration of the HIV response into the health sector, jeopardize the gains made in generation and use of evidence for planning. This dissertation makes a policy recommendation for incentives to sustain the use of evidence in HIV planning and policymaking. At the global level, these incentives could be created by including measures of evidencebased planning in international benchmarks of governance (Chapter 3). The instrument introduced in this dissertation to measure evidence quality and use in planning could provide an input to grant allocation decisions by donors and development banks, as an indicator of effective decision-making and strength of monitoring and evaluation performance. Application of the instrument before and after evidence generating activities could help identify barriers and enablers to evidence use at the national level.

The criteria emerging from the systematic review of literature in Chapter 2 illustrate the success of the HIV response in framing the public health and emergency response approach to the epidemic, within the context of social 
justice and development. As integration of the HIV response with the health sector is pursued, the policies and practices of integration must remain accountable to the key criteria of the HIV response. Integration of HIV into the health sector also implies some elements of the HIV monitoring system may become weaker in the process, while the overall sustainability of the HIV and health response gains strength from integration. Policymakers should consider this trade-off between effectiveness and sustainability in integration of the HIV response into the preventive health sector.

While greater involvement of the community of people living with or at risk of HIV in planning is recommended (Chapter 4 and 5), this dissertation recognizes that the problems and solutions of public health must be jointly owned by the government and the stakeholders. Findings of this dissertation demonstrate that the degree of agreement between civil society and government in prioritized criteria around HIV program choices creates an opportunity for policy advocacy for greater involvement of civil society in decision-making (Chapter 5). The dichotomy of views of stakeholders on criteria for prioritization in scientific and technical decisions, underlines the importance of investments in technical literacy of community based organizations to strengthen their role in decision-making or grass-roots movements for community driven policies in research, science and technology (Chapter 4). Discrete choice experiments with program decisions in Chapter 5 demonstrate that theoretical models, and not empirical evidence, drive HIV program managers' priorities. These findings imply that policies should be put in place to ensure accountability for programs that are based on the models. 


\section{Acknowledgements}

I am sincerely grateful to my advisor Prof. Wim Groot, for his continuous support of my PhD study, for his patience, mentoring, and encouragement, his wisdom, and humor that made our bi-monthly calls a pleasure. And to my coadvisor, Dr. Milena Pavlova, who read and listened to everything I had to say with immense interest and attention, and guided me with insightful suggestions, but also with hard questions which spurred me to broaden my perspective and venture into territories beyond my comfort. Asking them to be my advisers was the best decision I made. I cannot imagine having a better advisory team for my $\mathrm{PhD}$ study.

Besides my advisor, I would like to thank the rest of my thesis committee, Prof. Franziska Gassmann, Prof. Rob Baltussen, Prof. Silvia Evers, Prof. Gerjo Kok, and Prof. Hans Maarse for their detailed review and careful recommendations.

My very special gratitude goes out to Mindel. She made the entire process, from application to defense easy and fun. I'm grateful to her for welcoming me to start my PhD journey in Maastricht, discovering this charming city, surrounded by immense history and vibrating with positive energy, where I also made lifelong friendships with Brenda, Mutinta, Paul, Shuan, Andrea, Nyasha, Sonila, Julia, Clovis, Luiz, Mohamed, Nozipho, Alexey and Camilo.

With a special thanks to the colleagues, friends and family who believed in me, gave me unwavering encouragement, and facilitated my $\mathrm{PhD}$ journey as I juggled full-time work with my studies: Alba Vilajeliu, Anna Yakusik, Annukka 
Lipponen, Chika Hayashi, Claudia Maier, Desmond Spruijt, Erik Blankinship, Keith Sabin, Kristan Schoultz, Marjorie Opuni, Miriam Sabin, Reza Safarnejad.

I am also grateful to Nga Nguyen, Phuong Huynh, and Dr. Vo Hai Son. They truly opened my eyes and mind to the heart of Vietnamese people and the HIV epidemic in Viet Nam, and the spirit, determination, and dreams that characterizes the best of their country. A special thanks goes to Nguyen Giang Son for helping with the front cover design of this book.

I am most grateful to my wife, Miho, for her unrelenting support and thoughtfulness. Writing this dissertation kept my mind at a distance at times, but she was always present to drive me past hundreds of decisions and indecisions per hour. I cannot separate her influence on me from the success of delivering this dissertation. In the end, she and our forthcoming child gave me the strength and speed I needed to complete this work.

This book is dedicated to my life coach, my eternal cheerleader who taught me that as important as curiosity and inspiration for gaining knowledge, is perseverance and delayed gratification. Who taught me that in the end courage is not much different than patience, except that it takes longer. And who imperceptibly encouraged me toward the two values true to all of life's difficulties and accolades: commitment and humility. Who led by example, raising two boys, seeing them through a revolution, a war, and three continents, all the while shielding them the best she could from stigma and discrimination. And after seeing them off to college, found the courage in herself to enlist in a technical training course, learn to drive, teach herself a new language, and get her first job to become independent. To my mother, Azam Hamidi. 


\section{Curriculum Vitae}

Ali Safarnejad was born on December 30, 1973 in Esfahan, Iran. He immigrated to the United States in 1986, where he received his formative education, including a Bachelor of Science degree in Electrical Engineering, a Master of Science in Management and Business Administration, and another Master of Science in International Development. His career with the United Nations started in 2001 with the Food and Agriculture Organization in Rome, Italy, where he worked on information systems to monitor global food security and crisis early warning systems. In 2007, he joined the Joint United Nations Programme on HIV and AIDS (UNAIDS) in Geneva, Switzerland, where he guided global development of information systems for monitoring, evaluating, and reporting on the HIV epidemic and response.

Since 2015, Ali has been stationed in the Country Office of UNAIDS in Hanoi, Viet Nam as the Senior Strategic Information Advisor. In this position, he has been responsible for providing technical advisory support to the Viet Nam Ministry of Health in monitoring and evaluation of the epidemic and response, introducing catalytic innovations in surveillance, and providing the strategic information needed to mount an effective response to the HIV epidemic. As interim Director of UNAIDS Country Office between November 2016 and May 2017, he has provided guidance on strategic funding decision to the Viet Nam Authority for HIV and AIDS Control, and the development partners working in the HIV response. He currently co-chairs an interagency management team involving deputies of all United Nations agencies present in Viet Nam, working toward a joint and coherent approach to achieving the Sustainable Development Goals. 


\section{Publications}

Safarnejad A, Nga TN, and Son VH. 2017. "Population Size Estimation of Men Who Have Sex with Men in Ho Chi Minh City and Nghe An Using Social App Multiplier Method." Journal of Urban Health, 94(3), 339-349, Retrieved (http://dx.doi.org/10.1007/s11524-016-0123-0).

Safarnejad A, Izazola-Licea JA. 2017. "Direct and indirect effects of enablers on HIV testing, initiation and retention in antiretroviral treatment and AIDS related mortality." PLoS ONE, 12(2), 1-15, Retrieved (https://doi.org/10.1371/journal.pone.0172569)

Erkkola T, Safarnejad A, Ongpin P, Frescura L, Aran C, Alfven T, Sabin M, Panakadan S. "Focusing on effective use of AIDS data: AIDSinfo." Abstract no. TUPE666. Poster session presented at: 19th International AIDS Conference. Washington, D.C., 22-27 July 2012.

Safarnejad A. 5 May 2006. "A Global Indicator Information System." Directions Magazine, Retrieved (http://www.directionsmag.com/entry/a-global-indicatorinformation-system/123150).

Safarnejad A. November 2006. "Easy as XML: a new way to communicate georeferenced indicator data". Geo-world Magazine, 19(11), 32-36. 


\section{UNU-MERIT/MGSoG Dissertation Series}

2017

Clovis Freire

Diversification and Structural

Economic Dynamics

UNU-MERIT/MGSoG Dissertation

Series № 191

Michael Verba

Innovation and Knowledge Dynamics:

Essays on the Knowledge Economy

UNU-MERIT/MGSoG Dissertation

Series № 190

Pui Hang Wong

The Hearts and Minds in Conflict and

Peace:The Economics of

Counterinsurgency and the Psychology

of Reconstruction

UNU-MERIT/MGSoG Dissertation

Series № 189

Brenda Yamba

Schooling Despite All Odds: Evidence

from Lesotho on Female Child Carers

who Stayed in School

UNU-MERIT/MGSoG Dissertation

Series № 188

2016

Yesuf Awel

Insurance for Growth; Empirical

Essays on Insurance Demand and

Impacts in Africa

UNU-MERIT Dissertation Series,

№ 108

Sheng Zhong

Moving towards An Energy Efficient

Future; Essays on Energy Efficiency,

Technology and Development

UNU-MERIT/MGSoG Dissertation

Series № 187

Julieta Marotta

Access to Justice and Legal

Empowerment of Victims of Domestic

Violence through Legal Organizations

in the City of Buenos Aires; A

Qualitative Empirical Legal Study

UNU-MERIT/MGSoG Dissertation

Series, № 186

Andrea Franco-Correa

On the Measurement of

Multidimensional Poverty as a Policy

Tool; Empirical Applications to Chile, Colombia, Ecuador and Peru

UNU-MERIT/MGSoG Dissertation

Series, № 185 
Tigist Mekonnen Melesse

Grow More Food using Fewer

Resources;

Agricultural Technology Adoption and Innovation Practices for Inclusive and

Sustainable Development

UNU-MERIT Dissertation Series, № 107

Eleni Yitbarek

Getting Ahead or left Behind?; Essays on Poverty Dynamics and Social Mobility in Africa

UNU-MERIT Dissertation Series, № 106

Thuy Dieu Nguyen

Firm-Level Theory and Evidence of

Corruption

UNU-MERIT Dissertation Series,

№ 105

Raquel Tsukada Lehman

Essays on Household Production with

Labor-Saving Technology

UNU-MERIT Dissertation Series,

№ 104

Eva Barteková

Multi-Problem Challenges for a

Renewable Future; Empirical Studies

on Competitive Disadvantages from

Electricity Price Differentials and

Mineral Supply Risk in an Open

Economy

UNU-MERIT Dissertation Series, № 103
Jocelyn Olivari

Entrepreneurial Traits and Innovation;

Evidence from Chile

UNU-MERIT Dissertation Series,

№ 102

Muhammad Shafique

Essays on the role of knowledge, RED, and Technology-based Firms in the

Evolution of Socio-techno-economic

System

UNU-MERIT Dissertation Series,

№ 101

Serdar Türkeli

Governance of Innovation Policy;

Empirical Studies on Applied Political

Economy by Multi-Methods Analysis

UNU-MERIT Dissertation Series,

№ 100

Ayokunu Adedokun

Pathways to Sustainable Peacebuilding

in Divided Societies; Lessons and

Experiences from Mozambique

MGSoG Dissertation Series, № 75

Luiz Rothier Bautzer

Organizing Concurrent Engineering

through ICT Platforms

Blueprinting Product Lifecycle

Management Platforms across

Disciplinary Agencies

MGSoG Dissertation Series, № 74 
Natalia Popova

Migration in the Periphery of the

European Union;

Determinants of Successful and

Sustainable Labour Market Integration of Return Migrants in Albania, Egypt, Moldova and Tunisia

MGSoG Dissertations Series, № 73

Richard A. Martina

Uncertainty and Resource Constraint in the Small Island Developing States;

Essays in Entrepreneurial Cognition

MGSoG Dissertations Series, № 72

Cécile Cherrier

The Expansion of Basic Social

Protection in Low-income Countries;

An Analysis of Foreign Aid Actors'

Role in the Emergence of Social

Transfers in Sub-Saharan Africa

MGSoG Dissertations series, № 71

Paul Caldron

The Tacit Bargain in Short-Term

Medical Missions; Why U.S.

physicians go and what it costs

MGSoG Dissertation Series, № 70

2015

Hibret Belete Maemir

Dissecting Aggregate Productivity;

International Integration and Growth with Heterogeneous Firms

UNU-MERIT Dissertation Series, № 96
Mahmut Kobal

Customs \& Excellence: A Comparative Approach on Administrative and Regulatory Compliance Perspectives of the EU-Turkey Customs Union

MGSoG Dissertation Series, № 69

Craig Loschmann

Essays on Conflict-related Migration and Development in the Case of Afghanistan

MGSoG Dissertations Series, № 68

Andrea Milan

Rural Livelihoods, Location and

Vulnerable Environments; Approaches

to Migration in Mountain areas of

Latin America

MGSoG Dissertation Series, № 67

Farida Lada

On Guarding the Welfare of Clinical

Trial Subjects While Promoting Novel

Drug Innovation

A Game Theoretical Approach

MGSoG Dissertation Series, № 66

Giorgio Triulzi

Looking for the Right Path; Technology

Dynamics, Inventive Strategies and

Catching-up in the Semiconductor

Industry

UNU-MERIT Dissertation Series, № 95 
Abdul Baseer Qazi

Knowledge flows and networks in the

ICT sector; The case of Pakistan

UNU-MERIT Dissertation Series, № 94

Ajay Thutupalli

Technology Paradigm Shifts in

Agriculture; Drivers of Sustainability

and Catch up

UNU-MERIT Dissertation Series,

№ 93

Eduardo Urias

Improving access to HIVIAIDS

treatment in Brazil; When are

Compulsory Licenses effective in Price

Negotiations?

UNU-MERIT Dissertation Series, № 92

Francesca Guadagno

Why have so few Countries

Industrialised?

UNU-MERIT Dissertation Series, № 91

Daniel Opolot

The Evolution of Beliefs and Strategic

Behaviour

UNU-MERIT Dissertation Series, № 90

Alejandro Lavopa

Structural Transformation and

Economic Development; Can

Development Traps be Avoided

UNU-MERIT Dissertation Series, № 89
Jinjin Zhao

Urban water management reform; The

Case of China

UNU-MERIT Dissertation Series,

№ 88

Simona Vezzoli

Borders, Independence and Post-

colonial Ties; the Role of the State in

Caribbean Migration

MGSoG Dissertation Series, № 65

Silvia Consuelo Gómez Soler

Civil Conflict and Education; How

Does Exposure to Civil Conflict Affect

Human Capital Accumulation?

Evidence from Standardized Exit

Exams in Colombia

MGSoG Dissertation Series, № 64

Paula Nagler

Occupational Choice in the Developing

World

MGSoG Dissertation Series, № 63

Jasmin Kientzel

Determinants of Professional

Commitment to Environmental

Sustainability

MGSoG Dissertation Series, № 62

Mehmet Güney Celbiş

Regional Policies; Convergence, Trade,

and the Allocation of Public Capital

MGSoG Dissertation Series, № 61 
Florian Henning

Living Up to Standard;

Interoperability Governance and

Standards Adoption in Government

Information Networks

MGSoG Dissertation Series, № 60

Niels P. Groen

The Never-Ending Project

Understanding E-Government Project

Escalation

MGSoG Dissertation Series, № 59

Derek Copp

Teacher-Based Reactivity to Provincial

Large-scale Assessment in Canada

MGSoG Dissertation Series, № 58

Michaella Vanore

Family-Member Migration and the

Psychosocial Health Outcomes of

Children in Moldova and Georgia

MGSoG Dissertation Series, № 57

2014

Dirk Crass

The Impact of Brands on Innovation and Firm Performance; Empirical

Evidence from Germany

UNU-MERIT Dissertation Series, № 87

Samyukta Bhupatiraju

The Geographic Dimensions of Growth and Development

UNU-MERIT Dissertation Series, № 86
Sonja Fransen

The Economic and Social Effects of

Remittances and Return Migration in

Conflict-Affected Areas; The Case of

Burundi

MGSoG Dissertation Series, № 56

Ibrahim Khalil Conteh

The Impact of Floods on Primary

School Education in Zambia

MGSoG Dissertation Series, № 55

Richard Bluhm

Growth Dynamics and Development

Essays in Applied Econometrics and

Political Economy

MGSoG Dissertation Series, № 54

Nevena P. Zhelyazkova

Work-Family Reconciliation and Use of

Parental Leave in Luxembourg;

Empirical Analysis of Administrative

Records

MGSoG Dissertation Series, № 53

François Lafond

The Evolution of Knowledge Systems

UNU-MERIT Dissertation Series,

№ 85

Annalisa Primi

Promoting Innovation in Latin

America; What Countries Have

Learned (and What They Have Not) in

Designing and Implementing

Innovation and Intellectual Property

Policies

UNU-MERIT Dissertation Series, № 84 
Fatoumata Lamarana Diallo

Evaluation of Meal and Deworming

Programs for Primary Schools in Rural

Senegal

UNU-MERIT Dissertation Series, № 83

Sachin Kumar Badkas

Metachoice and Metadata; Innovating with Environmental Policy Analysis in Europe

MGSoG Dissertation Series, № 52

Irina S. Burlacu

An Evaluation of Tax-Benefit Systems Impact on the Welfare of Frontier

Worker;

The Case of Luxembourg and Belgium MGSoG Dissertation Series, № 51

Özge Bilgili

Simultaneity in Transnational

Migration Research; Links Between

Migrants' Host and Home Country

Orientation

MGSoG Dissertation Series, № 50

Yulia Privalova Krieger

Reshaping the Big Agenda;

Transnational Politics and Domestic

Resistance Financial crisis and social protection reform in Bosnia and

Herzegovina

MGSoG Dissertation Series, № 49

Marieke van Houte

Moving Back or Moving Forward?

Return migration after Conflict

MGSoG Dissertation Series, № 48
Oxana Slobozhan

Global Governance in the Management of Natural Resources; The Case of the Extractive Industries Transparency Initiative (EITI)

MGSoG Dissertation Series, № 47

Luis Bernardo Mejia Guinand

The Changing Role of the Central

Planning Offices in Latin America; A

Comparative Historical Analysis

Perspective (1950-2013)

MGSoG Dissertation Series, № 46

Cheng Boon Ong

Ethnic Segregation in Housing,

Schools and Neighbourhoods in the

Netherlands

MGSoG Dissertation Series, № 45

Luciana V. Cingolani

Bureaucracies for Development;

Oxymoron or Reality? Studies on State

Capacity in Challenging Governance

Contexts

MGSoG Dissertation Series, № 44

Carlos Cadena Gaitán

Green Politics in Latin American

Cities - Sustainable Transport Agendas

MGSoG Dissertation Series, № 43

Katie Kuschminder

Female Return Migration and

Reintegration Strategies in Ethiopia

MGSoG Dissertation Series, № 42

Metka Hercog

Highly-Skilled Migration and New

Destination Countries

MGSoG Dissertation Series, № 41 
Margaret Agaba Rugadya

Can Remittances Influence the Tenure and Quality of Housing in Uganda?

MGSoG Dissertation Series, № 40

\section{3}

Anant Kamath

Information Sharing through Informal

Interaction in Low-Tech Clusters

UNU-MERIT Dissertation Series, № 82

Flavia Pereira de Carvalho

What we talk about when we talk about

Brazilian Multinationals; An

Investigation on Brazilian FDI,

Economic Structure, Innovation and

the Relationship between them

UNU-MERIT Dissertation Series,

№ 81

Jun Hou

Complementarity in Innovation and

Development; A Cross-country

Comparison

UNU-MERIT Dissertation Series, № 80

Rufin Baghana

Impacts of Government Incentives to $R \mathcal{E D}$, Innovation and Productivity;

A Micro econometric Analysis of the Québec Case

UNU-MERIT Dissertation Series, № 79
Ilire Agimi

New Governance Under Limited

Statehood; The Case of Local

Government Reform in Kosovo

MGSoG Dissertation Series, № 39

\section{Lilia I. Stubrin}

High-Tech Activities in Emerging

Countries; A Network perspective on

the Argentinean Biotech Activity

UNU-MERIT/MGSoG Dissertation

Series, № 78

Kristine Farla

Empirical Studies on Institutions,

Policies and Economic Development

MGSoG Dissertation Series, № 38

Marina Petrovic

Social Assistance and Activation in the

Pursuit of Happiness; Shedding New

Light on Old Policy Solutions to Social

Exclusion

MGSoG Dissertation Series, № 37

Laura Torvinen

Assessing Governance Assessments;

The Case of Mozambique; Governance

Assessments in the Context of Aid

Effectiveness Discourse

MGSoG Dissertation Series, № 36

Biniam Egu Bedasso

Institutional Change in the Long

Shadow of Elite; Essays on

Institutions, Human Capital and

Ethnicity in Developing Countries

MGSoG Dissertation Series, № 35 
Sepideh Yousefzadeh Faal Deghati Childhoods Embargoed; Constructing and Reconstructing Multidimensional Child Poverty in Iran 1984-2009

MGSoG Dissertation Series, № 34

Robert Bauchmüller

Investing in Early Childhood Care and Education; The Impact of Quality on Inequality

MGSoG Dissertation Series, № 33

\section{2}

Abdul Waheed

Innovation Determinants and

Innovation as a Determinant; Evidence

from Developing Countries

UNU-MERIT Dissertation Series, № 77

Bilal Mirza

Energy Poverty and Rural Energy

Markets in Pakistan

UNU-MERIT Dissertation Series,

№ 76

Benjamin Engelstätter

Enterprise Software and Video Games;

An Empirical Analysis

UNU-MERIT Dissertation Series,

№ 75

Fulvia Farinelli

Natural Resources, Innovation and

Export Growth; The Wine Industry in Chili and Argentina

UNU-MERIT Dissertation Series
Martin Rehm

Unified Yet Separated; Empirical

Study on the Impact of Hierarchical

Positions within Communities of

Learning

MGSoG Dissertation Series, № 32
Rodolfo Lauterbach

Innovation in Manufacturing; From

Product Variety and Labor

Productivity Growth to Economic

Development in Chile

UNU-MERIT Dissertation Series

Kirsten Wiebe

Quantitative Assessment of

Sustainable Development and Growth in Sub-Saharan Africa

UNU-MERIT/MGSoG Dissertation Series, № 74

Julio Miguel Rosa

Organizational Strategies, Firms'

Performance and Spatial Spillovers;

The Canadian Case in Research and

Development.

UNU-MERIT Dissertation Series, № 73

Johannes Wilhelmus Marie Boels Joseph Schumpeter, Honderd Jaar Economische Ontwikkeling; Een Historisch-theoretische Beschouwing. UNU-MERIT Dissertation Series 
Dorcas Mbuvi

Utility Reforms and Performance of the

Urban Water Sector in Africa

MGSoG Dissertation Series, № 31

Lina Salanauskaite

Distributional Impacts of Public

Policies; Essays in Ex-Ante and Ex-

Post Evaluation

MGSoG Dissertation Series, № 30

Esther Schüring

To Condition or not - is that the

Question?

An Analysis of the Effectiveness of Ex-

Ante and Ex-Post Conditionality in

Social Cash Transfer Programs

MGSoG Dissertation Series, № 29

\section{1}

Daniel Vertesy

Interrupted Innovation; Emerging

Economies in the Structure of the

Global Aerospace Industry

UNU-MERIT Dissertation Series,

№ 72

Tina Saebi

Successfully Managing Alliance

Portfolios; An Alliance Capability

View

UNU-MERIT Dissertation Series, № 71

Nora Engel

Tuberculosis in India; A Case of

Innovation and Control

UNU-MERIT/MGSoG Dissertation

Series, № 70
Joe Abah

Strong Organisations in Weak States;

Atypical Public Sector Performance in

Dysfunctional Environments

MGSoG Dissertation Series, № 28

Zina Samih Nimeh

Social Citizenship Rights; Inequality

and Exclusion

MGSoG Dissertation Series, № 27
Evans Mupela

Connectivity and growth in Sub-

Saharan Africa; The Role of

Communication Satellites

UNU-MERIT Dissertation Series, № 69

Nantawan Kwanjai

Cross Cultural Intelligence amid

Intricate Cultural Webs; A Tale of the

UnDutchables in the Land of 1002

Smiles

UNU-MERIT Dissertation Series, № 68 
Lina Sonne

Innovation in Finance to Finance

Innovation; Supporting Pro-poor

Entrepreneur-based Innovation

UNU-MERIT Dissertation Series,

№ 67

Lenka Eisenhamerová

Legitimacy of 'Humanitarian Military

Intervention'

MGSoG Dissertation Series, № 26

Sonila Tomini

Informal Payments for Health Care

Services in Albania

MGSoG Dissertation Series, № 25

Jinjing Li

Dynamic Microsimulation in Public

Policy Evaluation

MGSoG Dissertation Series, № 24

Aziz Atamanov

Rural Nonfarm Employment and

International Migration as

Alternatives to Agricultural

Employment; The Case of Kyrgyzstan

MGSoG Dissertation Series, № 23

Frieda Vandeninden

Poverty Alleviation; Aid and Social

Pensions

MGSoG Dissertation Series, № 22
Juliana Nyasha Tirivayi

The Welfare Effects of Integrating

AIDS Treatment with Food Transfers;

Evidence from Zambia

MGSoG Dissertation Series, № 21

Agnieska Ewa Sowa

Who's Left Behind? Social Dimensions

of Health Transition and Utilization of

Medical Care in Poland

MGSoG Dissertation Series, № 20

Emmanaouil Sfakianakis

The Role of Private Actors in the

Provision of Public Goods with

Applications to Infrastructure and

Financial Stability

MGSoG Dissertation Series, № 19

Siu Hing Lo

White Collars Green Sleeves; An Inter-

organizational Comparison of

Determinants of Energy-Related

Behaviors among Office Workers

MGSoG Dissertation Series, № 18

Treena $\mathrm{Wu}$

Constraints to Human Capital

Investment in Developing Countries;

Using the Asian Financial Crisis in

Indonesia as a Natural Experiment

MGSoG Dissertation Series, № 17

Henry Espinoza Peña

Impact Evaluation of a Job-Training

Programme for Disadvantaged Youths;

The Case of Projoven

MGSoG Dissertation Series, № 16 
Fernando Santiago

Human Resources Management

Practices and Learning for Innovation in Developing Countries;

Pharmaceutical Firms in Mexico

UNU-MERIT Dissertation Series, № 66

Zakaria Babutsidze

Essays on Economies with

Heterogeneous Interacting Consumers

UNU-MERIT Dissertation Series, № 65

Bertha Vallejo

Learning and Innovation Under

Changing Market Conditions; The

Auto Parts Industry in Mexico

UNU-MERIT Dissertation Series,

№ 64

Donatus Ayitey

Technical Change, Competitiveness and Poverty Reduction; A Study of the Ghanaian Apparel Industry

UNU-MERIT Dissertation Series, № 63

Sergey Filippov

Multinational Subsidiary Evolution;

Corporate Change in New EU Member States

UNU-MERIT Dissertation Series, № 62
Asel Doranova

Technology Transfer and Learning under the Kyoto Regime; Exploring the Technological Impact of CDM Projects in Developing Countries

UNU-MERIT Dissertation Series, № 61

Florian Tomini

Between Family and Friend;

Understanding the Interdependency of Private Transfers

MGSoG Dissertation Series, № 15

Michał Polalowski

The Institutional Transformation of

Social Policy in East Central Europe;

Poland and Hungary in Comparative

and Historical Perspective

MGSoG Dissertation Series, № 14

Maha Ahmed

Defining, Measuring and Addressing

Vulnerability; The Case of Post

Conflict Environments

MGSoG Dissertation Series, № 13

Pascal Beckers

Local Space and Economic Success; The

Role of Spatial Segregation of Migrants in the Netherlands

MGSoG Dissertation Series, № 12

Victor Cebotari

Conflicting Demands in Ethnically

Diverse Societies; Ethno political

Contention and Identity Values in

Europe

MGSoG Dissertation Series, № 11 
Dennis Gyllensporre

Competing and Complementary

Perspectives on the EU as a Crisis

Management Actor;

An Examination of the Common

Security and Defence Policy through

the Lenses of Idealism and Realism

MGSoG Dissertation Series, № 10

Judit Vall Castello

Business Cycle and Policy Effects on

Labour Market Transitions of Older

and Disabled Workers in Spain

MGSoG Dissertation Series, № 9

Keetie Roelen

False Positives or Hidden Dimensions;

The Definition and Measurement of

Child Poverty

MGSoG Dissertation Series, № 8

2009

Alexis Habiyaremye

From Primary Commodity Dependence

to Diversification and Growth;

Absorptive Capacity and Technological

Catch Up in Botswana and Mauritius.

UNU-MERIT Dissertation Series,

№ 60

Yoseph Getachew

The Role of Public Capital in Economic

Development

UNU-MERIT Dissertation Series,

№ 59
Denisa Maria Sologon

Earning Dynamics in Europe

MGSoG Dissertation Series, № 7

Melissa Siegel

Money and Mobility; Migration and

Remittances

MGSoG Dissertation Series, № 6

Jessica S. Hagen-Zanker

Modest Expectations; Causes and

Effects of Migration on Migrant

Households in Source Countries

MGSoG Dissertation Series, № 5
Sandra Leitner

Embodied Technological Change and Patterns of Investment in Austrian

Manufacturing

UNU-MERIT Dissertation Series, № 58

Semih Akçomak

The Impact of Social Capital on

Economic and Social Outcomes

UNU-MERIT Dissertation Series, № 57

Abraham Garcia

The Role of Demand in Technical

Change

UNU-MERIT Dissertation Series, № 56 
Saurabh Arora

Coherence in Socio-technical Systems;

A Network Perspective on the

Innovation Process

UNU-MERIT Dissertation Series, № 55

Mirtha R. Muniz Castillo

Human Development and Autonomy in Project Aid; Experiences from four bilateral projects in Nicaragua and $E l$ Salvador

MGSoG Dissertation Series, № 4

2008

Rutger Daems

Medicines for the Developing World UNU-MERIT Dissertation Series, № 54

Johannes Hanel

Assessing Induced Technology;

Sombart's Understanding of Technical

Change in the History of Economics

UNU-MERIT Dissertation Series,

№ 53

Rifka Weehuizen

Mental Capital; the Economic

Significance of Mental Health

UNU-MERIT Dissertation Series,

№ 52
Christiane Arndt

Governance Indicators

MGSoG Dissertation Series, № 3

Britta Augsburg

Microfinance; Greater Good or Lesser

Evil?

MGSoG Dissertation Series, № 2
Danielle Cloodt

The Relationship between RED

Partnership Formation, Social

Embeddedness and Innovative

Performance

UNU-MERIT Dissertation Series, № 51

Sabine Fuss

Sustainable Energy Development

under Uncertainty

UNU-MERIT Dissertation Series, № 50

Geranda Notten

Measuring and Managing Poverty

Risks

MGSoG Dissertation Series, № 1 
Tobias Kronenberg

Reconciling Environmental

Conservation with Economic

Prosperity; The Feasibility of Double

Dividends in the Short and Long Run

UNU-MERIT Dissertation Series,

№ 49

Viktoria Kravtsova

Assessing the Impact of Foreign Direct

Investment in Transition Economies

UNU-MERIT Dissertation Series, № 48

2006

Bulat Sanditov

Essays on Social Learning and

Imitation

UNU-MERIT Dissertation Series, № 46

Mamata Parhi

Dynamics of New Technology

Diffusion; A Study of the Indian

Automotive Industry

UNU-MERIT Dissertation Series, № 45

Andreas Reinstaller

Social Structures and the Innovation

Process; Their Role in the Demand of

Firms and Consumers

UNU-MERIT Dissertation Series, № 44
Suhail Sultan

The Competitive Advantage of Small and Medium Sized Enterprises; The Case of Jordan's Natural Stone Industry UNU-MERIT Dissertation Series, № 47

Rose Kiggundu

Innovation systems and Development;

The Journey of a Beleaguered Nile

Perch Fishery in Uganda

UNU-MERIT Dissertation Series, № 43

Thomas Pogue

The Evolution of Research

Collaboration in South African Gold

Mining; 1886-1933

UNU-MERIT Dissertation Series, № 42

Geoffrey Gachino

Foreign Direct Investment, Spillovers and Innovation; The Case of Kenyan Manufacturing Industry

UNU-MERIT Dissertation Series, № 41 
Önder Nomaler

Technological Change, International

Trade and Growth; An Evolutionary,

Multi-Agents-Based Modeling

Approach

UNU-MERIT Dissertation Series,

№ 40

2005

Samia Satti Osman Mohamed-Nour

Change and Skill Development in the

Arab Gulf Countries

UNU-MERIT Dissertation Series,

№ 39

Elad Harison

Intellectual Property Rights;

Economics and Policy Analysis

UNU-MERIT Dissertation Series, № 38

Daniel Dalohoun

The Relationship between RED

Partnership Formation, Social

Embeddedness and Innovative

Performance; a Multi-level Approach of Social Embeddedness

UNU-MERIT Dissertation Series, № 37

Müge Ozman

Networks, Organizations and

Knowledge

UNU-MERIT Dissertation Series,

№ 36
Bas Straathof

Product Variety and Economic

Growth; The Counteracting Effects of

Scale and Idiosyncrasy

UNU-MERIT Dissertation Series,

№ 35

Wilfred Schoenmakers

Knowledge Flows between

Multinational Companies; A Patent

Data Analysis

UNU-MERIT Dissertation Series,

№ 34

Myriam Cloodt

Mergers and Acquisitions ( $M$ and As)

in High-Tech Industries; Measuring

the Post-M and A Innovative

Performance of Companies

UNU-MERIT Dissertation Series, № 33 
Paola Criscuolo

$R \mathcal{E D}$ Internationalisation and

Knowledge Transfer; Impact on MNEs and their Home Countries

UNU-MERIT Dissertation Series, № 32

Maarten Verkerk

Trust and Power on the Shop Floor UNU-MERIT Dissertation Series, № 31
Gottfried Leibbrandt

Adoption, Harmonization and Succession of Network Technologies across Countries

UNU-MERIT Dissertation Series, № 30

Mark Sanders

Skill Biased Technical change; Its Origins, the Interaction with the Labour Market and Policy Implications UNU-MERIT Dissertation Series, № 29

Masaru Yarime

From End-of-Pipe Technology to Clean

Technology

UNU-MERIT Dissertation Series,

№ 26

Stéphane Malo

The Combinatorial Chemistry

Revolution; Sustaining a Superior

Performance Position through

Technological Learning

UNU-MERIT Dissertation Series,

№ 25

2002

Annelies Hogenbirk

Determinants of Inward Foreign

Direct Investment; the Case of the

Netherlands

UNU-MERIT Dissertation Series, № 24
Bastiaan Johan ter Weel

The Computerization of the Labour Market

UNU-MERIT Dissertation Series 
John Adeoti

Technology Investment in Pollution

Control in Sub-Saharan Africa; The

Case of the Nigerian Manufacturing Industry

UNU-MERIT Dissertation Series, № 23
Edward Huizenga

Innovation Management; How

Frontrunners Stay Ahead; An

Empirical Study on Key Success

Factors in the ICT sector

UNU-MERIT Dissertation Series,

№ 22

2000

Machiel van Dijk

Technological Change and the Dynamics of Industries; Theoretical Issues and Empirical evidence from Dutch Manufacturing

UNU-MERIT Dissertation Series, № 21

1999

Jan Cobbenhagen

Managing Innovation at the Company

Level; A Study on Non-Sector-Specific

Success Factors

UNU-MERIT Dissertation Series,

№ 20
Marjolein Caniëls

Regional Growth Differentials; The

Impact of Locally Bounded Knowledge

Spillovers

UNU-MERIT Dissertation Series,

№ 19

1998

\section{Aldo Geuna}

Resource Allocation and Knowledge production; Studies in the Economics of University Research

UNU-MERIT Dissertation Series, № 18

1996

Reinoud Joosten

Dynamics, Equilibria, and Values

UNU-MERIT Dissertation Series, № 17
Hugo Kruiniger

Investment, $R \mathcal{E} D$, and the Financing

Decisions of the Firm

UNU-MERIT Dissertation Series, № 16 
Hans van Meijl

Endogenous Technological Change;

The Case of Information Technology,

Theoretical Considerations and

Empirical Results

UNU-MERIT Dissertation Series,

№ 15

René Kemp

Environmental Policy and Technical

Change; A Comparison of the

Technological Impact of Policy

Instruments

UNU-MERIT Dissertation Series, № 14
Rohini Acharya

The Impact of New Technologies on

Economic Growth and Trade; A Case

Study of Biotechnology

UNU-MERIT Dissertation Series, № 13

Geert Duysters

The Evolution of Complex

Industrial Systems; The Dynamics of Major IT Sectors

UNU-MERIT Dissertation Series,

№ 12

\section{Marjan Groen}

Technology, Work and Organisation;

A Study of the Nursing Process in

Intensive Care Units

UNU-MERIT Dissertation Series, № 11

\section{4}

Huub Meijers

On the Diffusion of Technologies in a

Vintage Framework; Theoretical

Considerations and Empirical Results

UNU-MERIT Dissertation Series, № 10

Theon van Dijk

The Limits of Patent Protection; Essays

on the Economics of Intellectual

Property Rights

UNU-MERIT Dissertation Series,

№ 9
Hans Voordijk

Naar Integrale Logistiek in

Bedrijfsketens; Ontwikkelingen in de Bouw

UNU-MERIT Dissertation Series, № 8 
Paul Diederen

Technological Progress in Enterprises and Diffusion of Innovation;

Theoretical Reflections and Empirical Evidence

UNU-MERIT Dissertation Series, № 7

1992

Bart Verspagen

Uneven Growth Between

Interdependent Economies; An

Evolutionary View on Technology

Gaps, Trade and Growth

UNU-MERIT Dissertation Series, № 4

Sjoerd Romme

A Self-organization Perspective on

Strategy Formation

UNU-MERIT Dissertation Series, № 3
Ben Dankbaar

Economic Crisis and Institutional

Change; The Crisis of Fordism from the Perspective of the Automobile Industry UNU-MERIT Dissertation Series, № 6

Hanno Roberts

Accountability and Responsibility; The Influence of Organisation Design on Management Accounting

UNU-MERIT Dissertation Series, № 5 
John Spangenberg

Economies of Scale, and Atmosphere in Research Organisations

UNU-MERIT Dissertation Series, № 2

1988

John Hagedoorn

Evolutionary and Heterodox Innovation Analysis; A Study of Industrial and Technological Development in Process Control and Information Technology

UNU-MERIT Dissertation Series, № 1 
